\title{
Five insights from the Global Burden of Disease Study 2019
}

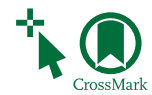

GBD 2019 Viewpoint Collaborators*

The Global Burden of Diseases, Injuries, and Risk Factors Study (GBD) 2019 provides a rules-based synthesis of the available evidence on levels and trends in health outcomes, a diverse set of risk factors, and health system responses. GBD 2019 covered 204 countries and territories, as well as first administrative level disaggregations for 22 countries, from 1990 to 2019. Because GBD is highly standardised and comprehensive, spanning both fatal and non-fatal outcomes, and uses a mutually exclusive and collectively exhaustive list of hierarchical disease and injury causes, the study provides a powerful basis for detailed and broad insights on global health trends and emerging challenges. GBD 2019 incorporates data from 281586 sources and provides more than 3.5 billion estimates of health outcome and health system measures of interest for global, national, and subnational policy dialogue. All GBD estimates are publicly available and adhere to the Guidelines on Accurate and Transparent Health Estimate Reporting. From this vast amount of information, five key insights that are important for health, social, and economic development strategies have been distilled. These insights are subject to the many limitations outlined in each of the component GBD capstone papers.

\section{Double down on catch-up development}

In the Global Burden of Diseases, Injuries, and Risk Factors Study (GBD), a population's social and economic development status for each location-year is tracked on the basis of the Socio-demographic Index (SDI), which combines information on gross domestic product per capita, average years of schooling among individuals aged older than 25 years, and the total fertility rate among females under the age of 25 years (as a widely available inverse proxy for the status of girls and women in society). ${ }^{1}$ SDI ranges from 0 to 100 . Since 1950 , global SDI has increased monotonically from 35 to 65 . The average pace of progress accelerated from 1950 to 1980 and has stayed at around 0.5 units per year since then. For the $15 \%$ of countries with the fastest rate of increase, SDI has

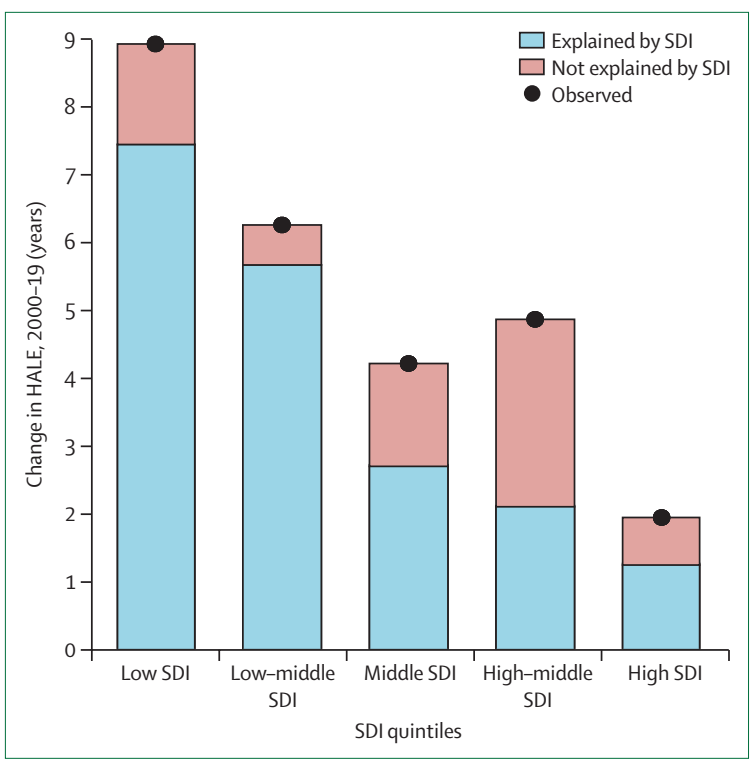

Figure 1: Change in HALE disaggregated by SDI quintiles, 2000-19

SDI quintiles as assessed in 2019. Expected change in HALE related to change in SDI is based on fitting spline functions to the relationship between age-specific mortality and SDI, and age-specific years lived with disability per capita and SDI. HALE=healthy life expectancy. SDI=Socio-demographic Index. improved on average by 0.9 units per year since 1980 , but it has improved by less than a third of this rate $(0.3$ units per year) for the bottom $15 \%$ of countries. Social and economic development can take centuries. Given what was reported from 1950 to 2019, the average country would take about 184 years to progress from an SDI of 0 to an SDI of 100; whereas, countries in the bottom $15 \%$ would take 357 years and those in the top $15 \%$ would take 110 years. From 1950 to 2000 , the pace of improvement in SDI was positively correlated with the level of SDI, whereby high and high-middle SDI countries developed faster than did low and low-middle SDI countries. Since 2000, the correlation has become progressively more negative and is now around -0.5 . In other words, since the Millennium Declaration, ${ }^{2}$ low and low-middle SDI countries have had larger annual increases in SDI than have high and high-middle SDI countries. The inequality in SDI between countries, measured with the standard deviation of SDI, has been decreasing since 2000, showing catch-up development.

Social and economic development, measured with SDI, is highly correlated with health outcomes. ${ }^{3}$ Figure 1 shows the increase in healthy life expectancy from 2000 to 2019, divided by what could be expected on the basis of SDI change alone and what is unexplained by SDI. These changes unexplained by SDI are likely to be explained by some combination of new technologies, prioritisation of societal resources for health, and the emergence of public health challenges, such as the HIV epidemic or alcohol consumption in eastern Europe and central Asia. Given the overwhelming impact of SDI on health progress, doubling down on policies and strategies that stimulate economic growth, expand access to primary and secondary schooling, and improve the status of women should be our collective priority. The catch-up social and economic development that has been clearly evident since the Millennium Declaration provides some optimism that maintaining focus on low SDI countries, and low SDI communities within countries, is not only possible but can also be expected to have profound health benefits. Further
Lancet 2020; 396: 1135-59

${ }^{*}$ Collaborators are listed at the end of the paper

Correspondence to: Prof Christopher J L Murray, Institute for Health Metrics and Evaluation, University of Washington, Seattle, WA 98195 , USA

cjlm@uw.edu

For GBD Compare see https://vizhub.healthdata.org/ gbd-compare/

For the GBD Results Tool see http://ghdx.healthdata.org/ gbd-results-tool

For more on the Guidelines on Accurate and Transparent Health Estimate Reporting see Statement Lancet 2016; 388: $19-23$ 


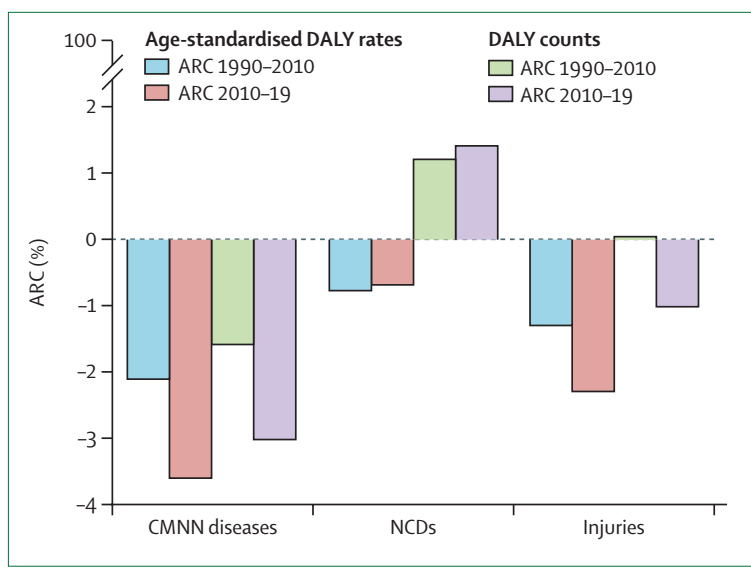

Figure 2: Global ARC for age-standardised DALY rates and DALY counts, 1990-2010 and 2010-19

Results are shown for three broad cause groups: CMNN diseases, NCDs, and injuries. $A R C=$ annualised rate of change. $C M N N=$ communicable, maternal, neonatal, and nutritional. DALY=disability-adjusted life-year. NCDs=non-communicable diseases.

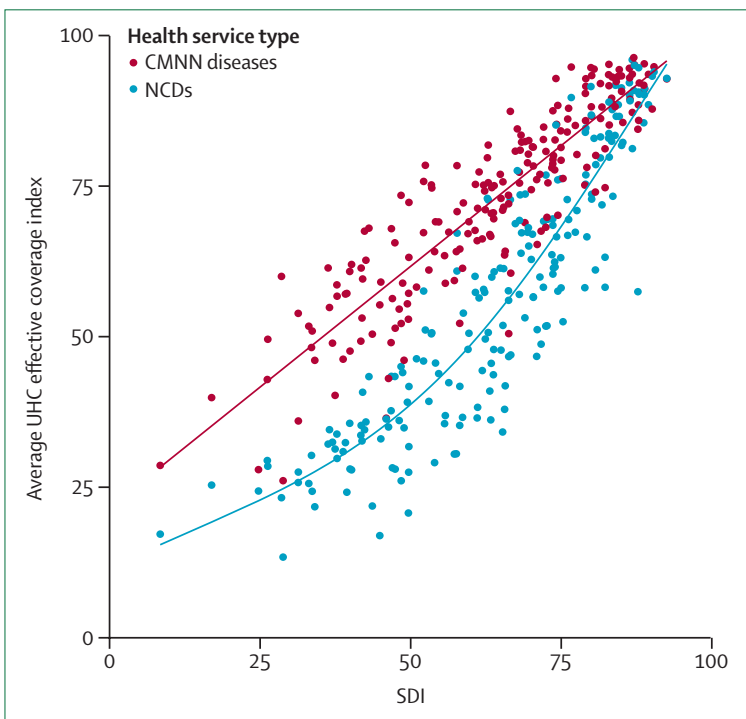

Figure 3: Relationships of UHC effective coverage in 204 countries and territories for health services targeting CMNN diseases and NCDs versus SDI, 2019

CMNN=communicable, maternal, neonatal, and nutritional. NCDs=noncommunicable diseases. SDI=Socio-demographic Index. UHC=universal health coverage.

improvements in health can also contribute to economic growth; higher educational attainment, because healthy children can learn more effectively; and greater female empowerment, as more women have access to reproductive health services, all of which create the potential for further health gains that expand SDI improvement.

\section{The Millennium Development Goal health agenda has been working}

Since around 2000, the global health community, including donors, has focused on reducing mortality of children and mothers, and the burden from three target conditions: tuberculosis, HIV, and malaria. ${ }^{2,4,5}$ Development Assistance for Health increased profoundly until 2010, but has since stagnated. ${ }^{6}$ The share of this assistance allocated to the Millennium Development Goal agenda has remained constant, even with the expanded Sustainable Development Goal health agenda in place since 2015. This concerted focus on communicable, maternal, neonatal, and nutritional (CMNN) diseases has led to faster progress in combatting these causes than has been reported for noncommunicable diseases (NCDs) and injuries (figure 2). Reductions in rates of age-standardised disability-adjusted life-years (DALYs) since 1990 have been largest for CMNN diseases and progress has been the fastest in the past decade, ${ }^{7}$ even considering the stagnation in Development Assistance for Health since 2010. Despite population growth, particularly in countries with the lowest SDI, the absolute number of DALYs from CMNN causes has also decreased. By contrast, age-standardised DALY rates for NCDs have barely reduced; in fact, population growth and ageing have resulted in a steady increase in the number of DALYs due to these causes. Age-standardised DALY rates for injuries have decreased, but at rates well below those of CMNN diseases.

\section{Health systems need to be more agile to adapt to the rapid shift to NCDs and disabilities}

Countries in the low-middle and middle SDI quintiles have had rapid transitions from disease burden dominated by CMNN causes to burden dominated by NCDs and injuries. The low-middle quintile increased from $37.8 \%$ of total DALYs caused by NCDs and injuries in 1990 to $66.0 \%$ in 2019 , with a similar pattern in the middle quintile as well. ${ }^{7}$ The GBD assessment of universal health coverage (UHC) allows disaggregation of coverage into interventions for CMNN diseases and NCDs (figure 3). ${ }^{8}$ Although coverage of CMNN diseases increases almost linearly with higher SDI, coverage of NCD intervention lags behind. Countries with rapid transitions have burden profiles dominated by NCDs, yet their health systems are struggling to deliver effective interventions for these diseases. As countries' health profiles shift to NCDs, there is a middle-SDI quintile gap that emerges, in which the most important interventions for improving health in these countries have low coverage. Despite this gap, most policy discussion, including WHO engagement with countries, is firmly focused on the agenda for CMNN diseases, ignoring the epidemiological heterogeneity of countries with low and middle SDI. The legacy focus on CMNN causes, and a failure to purposefully anticipate the inevitable shift to NCDs, is evident even in discussions on UHC. For example, the official UN UHC service indicator (3.8.1) does not include the coverage of any intervention targeting NCDs. ${ }^{9}$

A second challenge is emerging at high levels of development, in which most DALYs in high SDI countries 
now arise not from premature mortality, but from loss of functional health. This shift is driven by a combination of ageing populations and stagnant age-specific rates of years lived with disability (YLDs) for the main sources of functional health loss, including musculoskeletal disorders, mental disorders, substance misuse, vision loss, and hearing loss. The proportion of global DALYs due to YLDs increased from 20.7\% in 1990 to $33.9 \%$ in 2019.' Over the same period, the number of countries where YLDs exceeded years of life lost increased from one to 29 countries. This shift is mirrored in health expenditures in these high SDI nations. Musculoskeletal disorders were the largest health expenditure in the USA in 2016 at US $\$ 380$ billion, ${ }^{10}$ which was greater than expenditure for either cardiovascular diseases or cancers. Health systems appear to be poorly prepared for this shift. Most policy discussion is focused on cardiovascular diseases and cancers. ${ }^{11-13}$ The innovation pipeline for the main disabling conditions (musculoskeletal, mental, and neurological disorders) is poor. ${ }^{14-16}$ Similarly, low investment in research into underlying causes and therapeutic innovations for key causes of functional health loss is exacerbating this widespread and unacceptable neglect. For example, the US National Institutes of Health budget on cardiovascular diseases and cancers is dramatically larger than its budget on musculoskeletal disorders ( $\$ 8.60$ billion combined vs $\$ 734$ million in 2018). ${ }^{17}$

\section{Public health is failing to address the increase in crucial global risk factors}

The potential to improve health by risk reduction is well reported in GBD 2019. ${ }^{18}$ All risks quantified in GBD collectively account for $48 \%$ of global DALYs. Exposure to many risks highly correlated with SDI has been steadily decreasing as global SDI has increased, including household air pollution; child growth failure; and unsafe water, sanitation, and handwashing. Additionally, there have been notable decreases in exposure to smoking. Figure 4 shows the annualised rate of change in exposure from 2010 to 2019 for select risk factors ordered by global attributable DALYs. Among the 15 leading causes of attributable DALYs shown, high systolic blood pressure, high fasting plasma glucose, high body-mass index (BMI), ambient particulate matter pollution, alcohol use, and drug use stand out because rates of exposure are increasing by more than $0 \cdot 5 \%$ per year.

If public health action and public policy could stop or reverse the trends in exposure to these risks, the benefits would be huge. What lessons can be learned to improve health from humanity's collective failure to leverage knowledge on the harms associated with these risks? Simply providing information on the harms does not appear to be sufficient. The one major behavioural risk for adults is tobacco smoking, for which a cocktail of interventions built around strong commitment to government policy has had at least partial success. Smoking is down $1.2 \%$ per year globally since 2010 ,

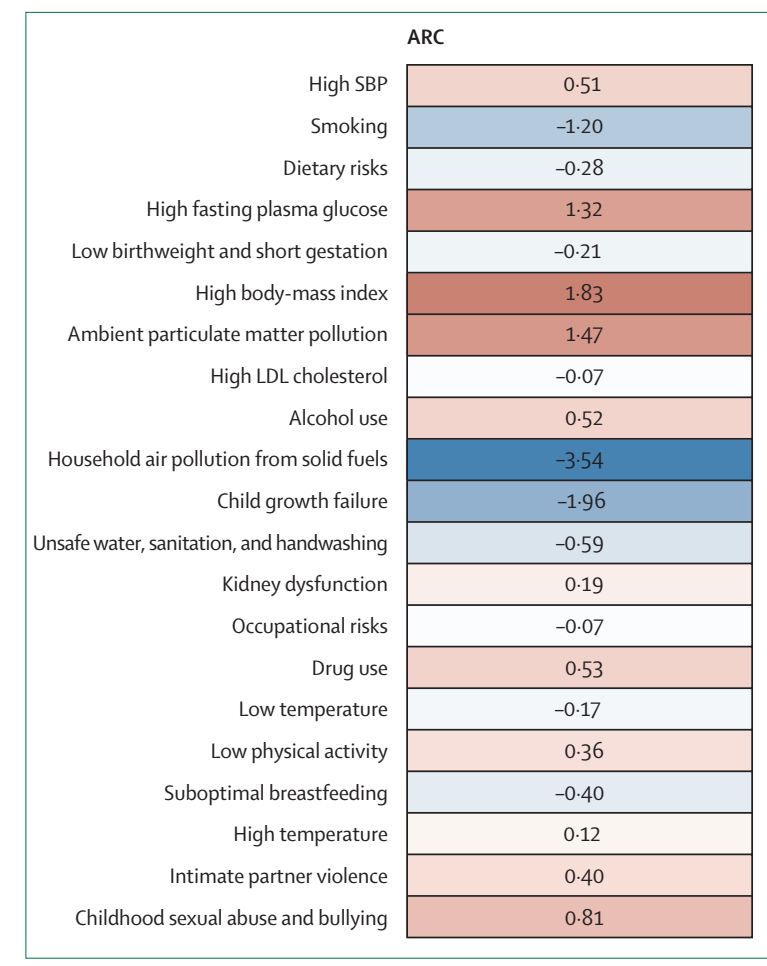

Figure 4: Global ARC in exposure for select risks, 2010-19 Risk factors are ordered by global attributable DALYs. Values are shaded by change in exposure, from dark blue (largest decrease) to dark red (largest increase). Exposure is measured as the age-standardised summary exposure value. Summary exposure value is an integrated measure of risk exposure that allows comparison across continuous, polytomous, and dichotomous risk factors. ARC=annualised rate of change. DALYs=disability-adjusted life-years. $\mathrm{SBP}=$ systolic blood pressure.

suggesting important lessons for strategies to reduce obesity, for example. This progress is more likely to be linked to taxation and legislation facilitated in part by the Framework Convention on Tobacco Contro ${ }^{19}$ than to providing information to consumers about the harms of tobacco, particularly in low and low-middle SDI countries. $^{20,21}$ The failure to slow or reverse the global rise in BMI, to facilitate healthier diets, or to increase amounts of physical activity is probably partly due to inadequate policy attention and funding for public health action and behavioural research. The steady rise of these risks is likely to pose a massive threat to future health progress everywhere. ${ }^{22,23}$ The increase in exposure to key metabolic risks and the slowing or reversals of long-term reductions in cardiovascular diseases seen in some locations suggest that the world might be approaching a turning point in terms of life expectancy gains. ${ }^{24}$ Governments should invest more funding in research and action to tackle these stagnating or worsening risk exposures. A core obstacle to accelerating progress on behavioural risks is the notion of individual agency and the need for governments to let individuals make their own choices. This concept is naive, given that individual choices are influenced by context, education, and availability of alternatives. 


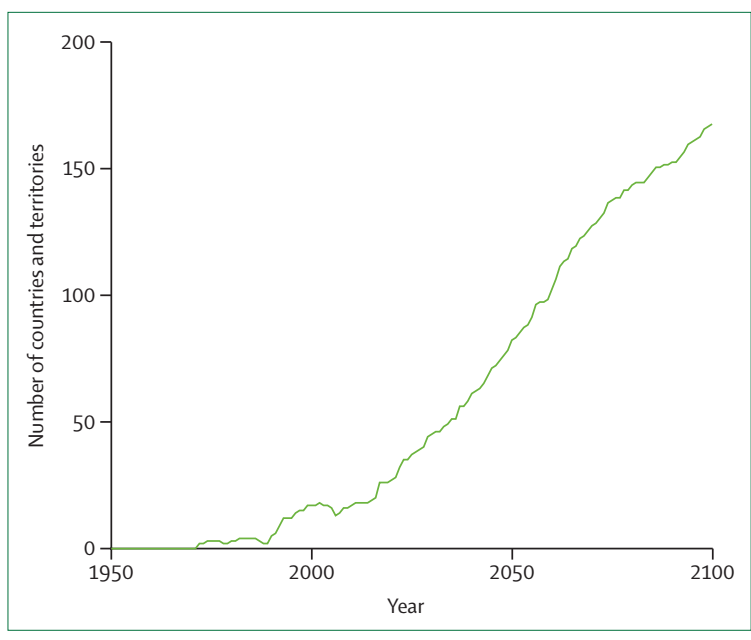

Figure 5: Number of countries and territories with negative natural rate of population increase, 1950-2019, and in the reference forecast scenario, 2020-2100

The natural rate of increase is negative when the crude death rate exceeds the crude birth rate.

Governments can and should take action to facilitate healthier choices by rich and poor individuals alike. When there is a major risk to population health, concerted government action through regulation, taxation, and subsidies, drawing lessons from decades of tobacco control, might be required to protect the public's health.

\section{Social, fiscal, and geopolitical challenges of inverted population pyramids}

As an extension of GBD, Vollset and colleagues ${ }^{25}$ have developed population scenarios for 195 countries and territories to 2100 . In 2019, 34 countries had negative natural rates of increase; in other words, the crude death rate was greater than the crude birth rate. When the natural rate of increase is negative, in the absence of net inmigration, populations will decrease. Two variables, female educational attainment and met contraceptive need, explain $80.5 \%$ of the variation in the completed fertility for a cohort of women. When met contraceptive need reaches $95 \%$ and average educational attainment reaches 16 years of age, the global total fertility rate will decrease to 1.41 . Globally, educational attainment is rising, as is contraceptive met need..$^{26,27}$ The effect of these trends will be a rapid increase in the number of countries with a negative natural rate of increase (figure 5). ${ }^{25}$ Over time, negative rates of natural increase progressively lead to inverted age pyramids, in which older 5-year age groups (eg, aged 40-44 years) are more populous than younger 5-year age groups (eg, aged 5-9 years). In 2050, China is projected to have 79.6 million people aged 70-74 years, but only $46 \cdot 0$ million children aged younger than 5 years. Negative natural rates of increase will lead to an intensified national debate on immigration. The profound social, fiscal, economic, and geopolitical challenges of an inverted age pyramid might best be addressed through liberal immigration policies. Countries such as Canada, Australia, New Zealand, and the USA have already used such a strategy to maintain or increase their working age populations in the face of decreasing fertility rates. Some countries will try to increase fertility rates rather than accept migrants into their societies. Russia has identified increasing the birth rate as their number one health priority. ${ }^{28}$ In such cases, it is imperative that any policy initiatives protect women's sexual and reproductive rights. Yet, attempts to increase fertility rates through economic incentives and paid maternal and paternal leave in countries like Sweden, ${ }^{27}$ Singapore, ${ }^{30}$ South Korea, ${ }^{31}$ Japan,,$^{32}$ and Taiwan (province of China) $)^{33}$ have had a minimal effect on fertility rates. ${ }^{25}$

\section{Conclusion}

Success in reducing the disease burden from causes of CMNN diseases by global collective action to fund key programmes should be celebrated. Catch-up social and economic development is fuelling more rapid health progress in the lower SDI quintiles. But there is reason to believe that, although the past 70 years have largely been a story of sustained improvements in health, rising exposure to crucial risks, such as high BMI, high fasting plasma glucose, and ambient particulate matter pollution, as well as stagnant exposure to many other behavioural risks, including diet quality and physical activity, might attenuate progress. Most alarmingly, the mortality decreases in cardiovascular diseases of the past half a century have slowed substantially, or even reversed, in some nations with high SDI. New challenges, such as rising temperature and the associated increases in poverty, need to be urgently addressed. Low fertility in many nations is likely to emerge as a profound social and economic challenge. Tracking progress across this myriad of global health challenges, and with health development goals more broadly, reinforces the policy value of global comparative assessments in the health sector, such as the ongoing GBD.

\section{Contributors}

Please see the appendix for more detailed information about individual authors' contributions to the research, divided into the following categories: managing the estimation process; writing the first draft of the manuscript; providing data or critical feedback on data sources developing methods or computational machinery; applying analytical methods to produce estimates; providing critical feedback on methods or results; drafting the work or revising it critically for important intellectual content; extracting, cleaning, or cataloguing data; designing or coding figures and tables; and managing the overall research enterprise.

\section{About the Global Burden of Disease Collaborator Network}

The Institute for Health Metrics and Evaluation (IHME) at the University of Washington engages a large network of individual collaborators with specialties in various topic areas to conduct the Global Burden of Diseases, Injuries, and Risk Factors Study (GBD) and its affiliated projects. GBD is a systematic, scientific effort to quantify the comparative magnitude of health loss due to diseases, injuries, and risk factors by age, sex, and geographies for specific points in time and is the largest and most comprehensive effort to date to measure

epidemiological levels and trends worldwide. Collaborators are crucial in 
both the data analysis as well as the policy uptake and proliferation of GBD and affiliated projects. Below, we list the individual collaborators who are authors on the publications listed in this issue.

\section{First and senior authors}

Five insights from the Global Burden of Disease Study 2019:

Christopher J L Murray (first author) and Alan D Lopez, Theo Vos, and Stephen S Lim (senior authors). Global age-sex-specific fertility, mortality, healthy life expectancy (HALE), and population estimates for 204 countries and territories, 1950-2019: a comprehensive demographic analysis for the Global Burden of Disease Study 2019: Haidong Wang (first author) and Christopher J L Murray (senior author). Global burden of 369 diseases and injuries in 204 countries and territories, 1990-2019: a systematic analysis for the Global Burden of Disease Study 2019: Theo Vos and Stephen S Lim (first authors) and Mohsen Naghavi and Christopher J L Murray (senior authors). Global burden of 87 risk factors in 204 countries and territories, 1990-2019: a systematic analysis for the Global Burden of Disease Study 2019: Christopher J L Murray, (first author), Aleksandr Aravkin, Peng Zheng, (ordered authors) and Michael Brauer, Ashkan Afshin, and Stephen S Lim (senior authors). Measuring universal health coverage based on an index of effective coverage of health services in 204 countries and territories, 1990-2019: a systematic analysis for the Global Burden of Disease Study 2019: Rafael Lozano (first author), Nancy Fullman,

John Everett Mumford, Megan Knight, and Celine Barthelemy (ordered authors) and Christopher J L Murray (senior author). Fertility, mortality, migration, and population scenarios for 195 countries and territories from 2017 to 2100: a forecasting analysis for the Global Burden of Disease Study: Stein Emil Vollset (first author), Emily Goren, Chun-Wei Yuan, Jackie Cao, Amanda E Smith, Thomas Hsiao, Catherine Bisignano (ordered authors), and Christopher J L Murray (senior author).

\section{Global Burden of Disease 2019 Collaborators}

Cristiana Abbafati*t] Mitra Abbasifard $\uparrow$, Mohsen Abbasi-Kangevari*十t

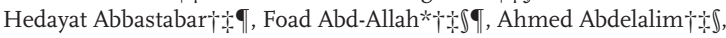

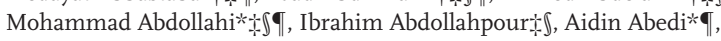

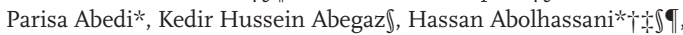
Akine Eshete Abosetugn $\uparrow$, Victor Aboyans*t』, Elissa M Abrams

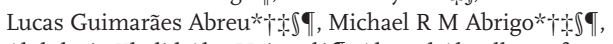
Abdulaziz Khalid Abu Haimed*ף, Ahmed Abualhasan』,

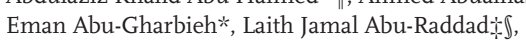

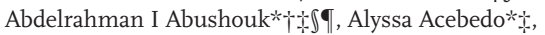
Ilana N Ackerman* + , Maryam Adabi $*+\lceil\llbracket$, Tim Adair $\uparrow$, Abdu A Adamu* $*$, Oladimeji M Adebayo*十+ $\uparrow$, Isaac Akinkunmi Adedeji†, Victor Adekanmbi† Jaimie D Adelson* $+\uparrow$, Abiodun Moshood Adeoye $\dagger \rrbracket$,

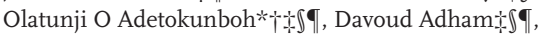
Shailesh M Advani† $\uparrow$, Mohsen Afarideh*, Mahdi Afsharił, Ashkan Afshin*十斤 Pradyumna Agasthi $\uparrow$, Kareha M Agesa $\neq$, Mohammad Aghaali $\uparrow$,

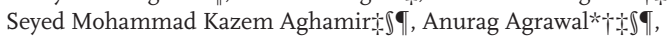

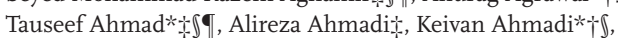
Mehdi Ahmadi*t], Hamid Ahmadieh $\uparrow+5$, Ehsan Ahmadpour Muktar Beshir Ahmed*†ฐ, Budi Aji*, Temesgen Yihunie Akaluł Rufus Olusola Akinyemitฐ

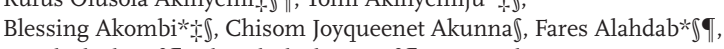

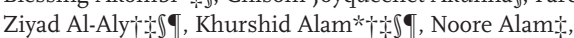

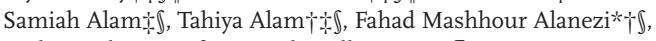

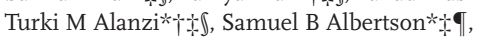

Jacqueline Elizabeth Alcalde-Rabanal†t, Niguse Meles Alema Biresaw Wassihun Alemu*』, Yihun Mulugeta Alemu*ף,

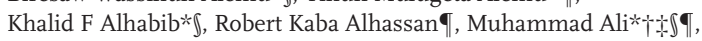

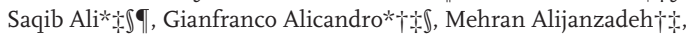

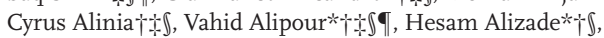

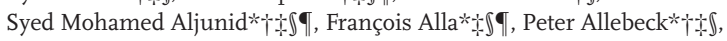
Majid Abdulrahman Hamad Almadi*广 $\uparrow$, Ali Almasi $\uparrow$,

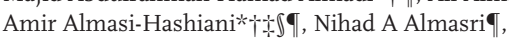

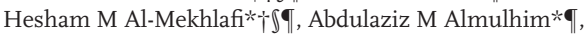

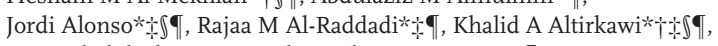
Arwa Khalid Alumran†, Nelson Alvis-Guzman*†† Nelson J Alvis-Zakzuk $\uparrow$, Azmeraw T Amare*, Bekalu Amare*, Saeed Amini* Mostafa Amini-Rarani $\uparrow$,
Fatemeh Amiritฐ

Dickson A Amugsi* $+\llbracket$ I, Gianna Gayle Herrera Amul $\$$,

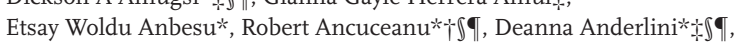

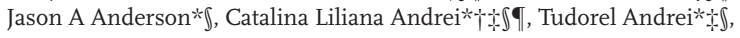
Sofia Androudi $\uparrow$, Colin Angus $\uparrow \rrbracket$, Mina Anjomshoa* $\uparrow+\downarrow$,

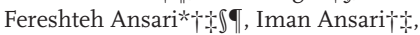

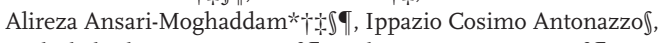

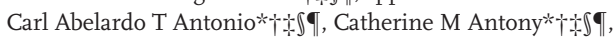

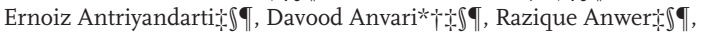
Seth Christopher Yaw Appiah*广』, Jalal Arabloo*广tฐ

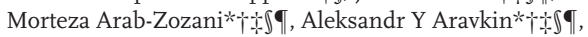
Aseb Arba Kinfe Arba*, Olatunde Aremu† Filippo Ariani $†$,

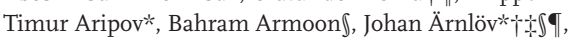
Oluwaseyi Olalekan Arowosegbe*, Krishna K Aryal ††,

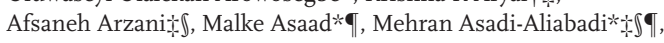

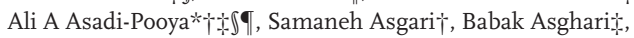
Mohammad Asghari Jafarabadi $\uparrow$, Charlie Ashbaugh* $* \int$, Michael Assmus*』, Zahra Atafar』, Seyyed Shamsadin Athari $\llbracket$,

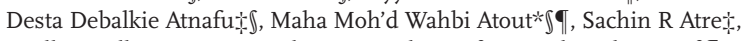

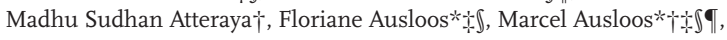
Leticia Avila-Burgos $\uparrow \uparrow$, Euripide Frinel Gbenato Arthur Avokpaho $\dagger$,

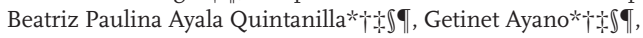

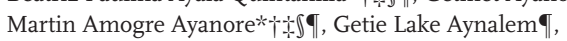

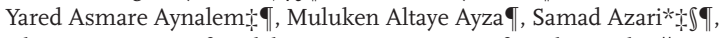
Ghasem Azarian $† \downarrow \rrbracket$, Zelalem Nigussie Azene* $+\int$, Gulrez Azhar||, Peter S Azzopardi $\boldsymbol{\uparrow}$, Darshan B B*ף, Ebrahim Babaee*十+

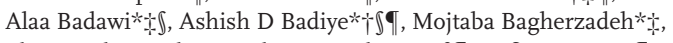
Eleni Bagli $\uparrow$, Mohammad Amin Bahrami $\uparrow \llbracket$, Atif Amin Baig $\uparrow$,

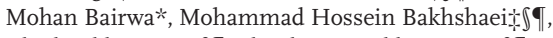

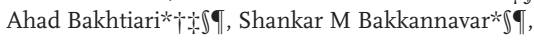
Arun Balachandran*ף, Senthilkumar Balakrishnan + , Shivanthi Balalla Shelly Balassyano $+\uparrow$, Alberto Baldasseroni』, Kylie Ball』,

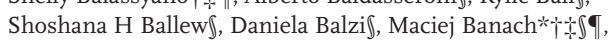

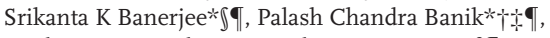
Marlena S Bannick Simachew Animen Bante $\llbracket$, Adhanom Gebreegziabher Baraki* $\uparrow$,

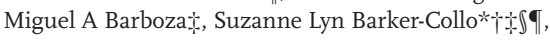
Till Winfried Bärnighausen $\uparrow \llbracket \uparrow$, Lope H Barrero』币,

Celine M Barthelemy

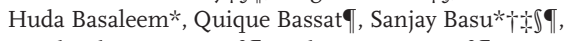

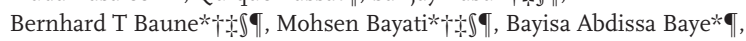

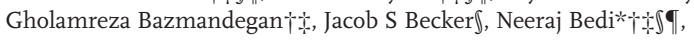

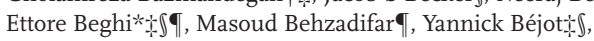
Tariku Tesfaye Tesfaye Bekuma $\uparrow$, Michelle L Bell $\uparrow_{\uparrow} \uparrow$, Aminu K Bello

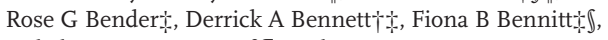
Isabela M Bensenor*广t

Kidanemaryam Berhe* $\uparrow+\downarrow \mathbb{\int}$, Adam E Berman* $\uparrow \rrbracket$, Eduardo Bernabe $+\uparrow$, Robert S Bernstein $\uparrow$, Gregory J Bertolacci $\uparrow$ t,

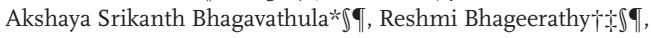

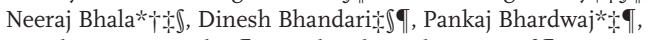
Anusha Ganapati Bhat $\uparrow$, Krittika Bhattacharyya*†t

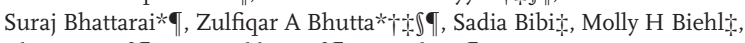

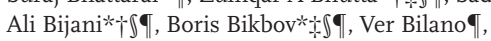

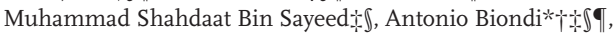
Binyam Minuye Birihane $+\widetilde{\Phi}$, Donal Bisanzio†t,

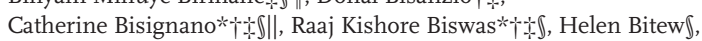

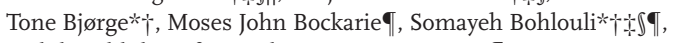

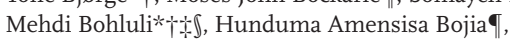
Srinivasa Rao Bolla*†+ Alexandra S Boon-Dooley $\neq$, Guilherme Borges $\$$, Antonio Maria Borzìt?d, Shiva Borzouei†』, Dipan Bose*, Cristina Bosettiף, Soufiane Boufous*ף, Rupert Bourne Oliver J Brady* †

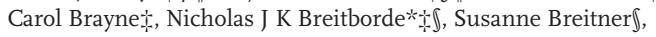

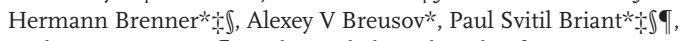
Andrew M Briggs $* \boldsymbol{q}$, Andrey Nikolaevich Briko $\uparrow$,

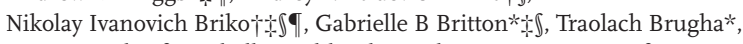

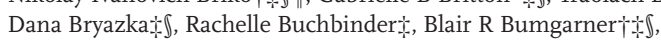
Katrin Burkartฐ, Richard Thomas Burnettฐ,

Sharath Burugina Nagaraja*tฐ ${ }^{*}$, Reinhard Busse $* \uparrow$,

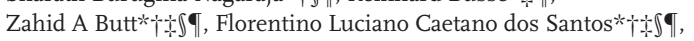


Leah E Cahill§, Lucero Cahuana-Hurtado $₫$, Tianji Cai†,

Charlton SKH Callender†, Luis Alberto Cámera*††

Ismael R Campos-Nonato*† + J , Julio Cesar Campuzano Rincon*,

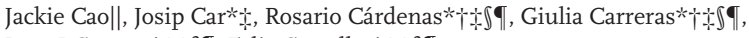

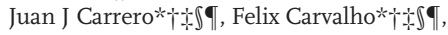

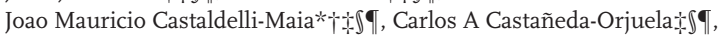

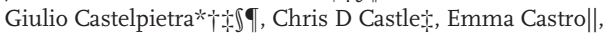

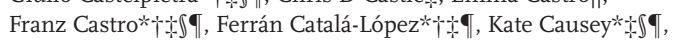

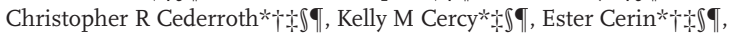

Julian Chalek\|, Joht Singh Chandan**厅

Angela Y Chang $₫$, Jung-Chen Chang*, Kai-Lan Changף,

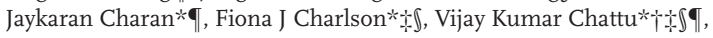

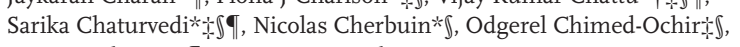

Ken Lee Chin*†⿰, Jesus Lorenzo Chirinos-Caceres*,

Daniel Youngwhan Cho*tฐ

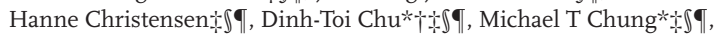

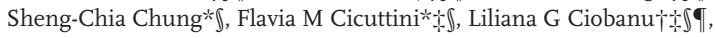

Massimo Cirillo*十斤 + , Beniamino Cislaghi*,

Thomas Khaled Dwayne Classen』, Aaron J Cohen*』, Emma L Collinst,

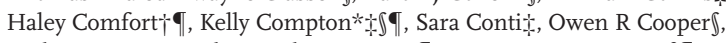

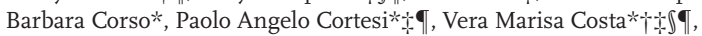

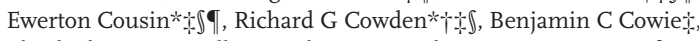
Elizabeth A Cromwell $\uparrow+$, Andrew J Croneberger $\uparrow$, Di H Cross + \, Marita Cross*, Christopher Stephen Crowe* $*$, Jessica A Cruz $\$$, Steven Cummins*, Matthew Cunningham $†$, Saad M A Dahlawi $\uparrow \leqslant \uparrow$, Haijiang Dai*, Hancheng Dai*, Albertino Antonio Moura Damasceno』, Giovanni Damiani*十斤

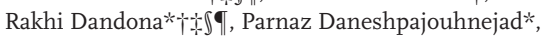

William James Dangel』, Anna-Karin Danielsson』, Jiregna Darega Gela $\llbracket$,

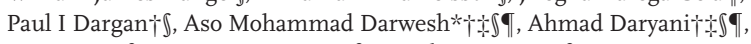

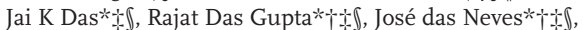
Aditya Prasad Dash $\uparrow$, Gail Davey*

Claudio Alberto Dávila-Cervantes*tฮ , Adrian C Davis*,

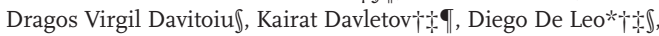
Jan-Walter De Neve $\boldsymbol{q}$, Frances E Dean $\neq$, Nicole K DeCleene

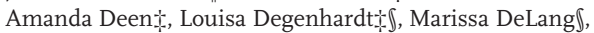
Robert Paul Dellavallețd, Feleke Mekonnen Demeketฐ, Gebre Teklemariam Demoz』, Desalegn Getnet Demsieț

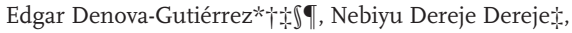

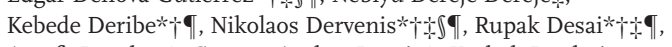
Assefa Desalewł, Getenet Ayalew Dessieł, Keshab Deuba*, Samath Dhamminda Dharmaratne $\uparrow$,

Govinda Prasad Dhungana*tr Diana Dias da Silva†』

Zahra Sadat Dibaji Forooshani $\$$, , Martin Dichgans*, Alireza Didarloo*, Zachary V Dingels

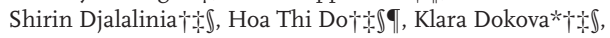
David Teye Doku†, Christiane Dolecek*, Andrew J Dolgert||,

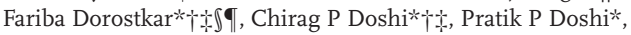

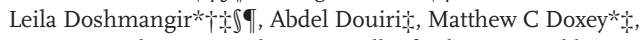
Kerrie E Doyle $\dagger$, Tim Robert Driscoll $* \$$, Eleonora Dubljanin $\dagger$,

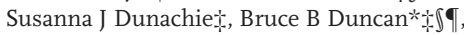

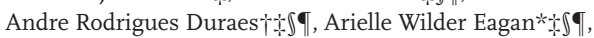
Hedyeh Ebrahimi*, Mohammad Ebrahimi Kalan*

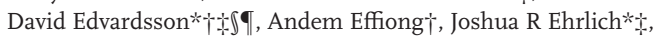
Nevine El Nahas

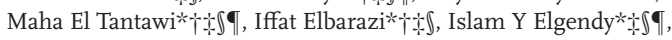
Hala Rashad Elhabashy Iqbal RF Elyazar* $\leftarrow$ I, Mohammad Hassan Emamian*†t,

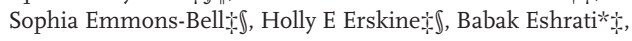
Khalil Eskandari $\uparrow$, Sharareh Eskandarieh*十† ${ }^{*}+$,

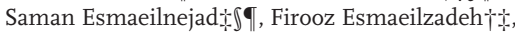

Alireza Esteghamati* $\leftarrow$, Sadaf Esteghamati*, Kara Estep $\uparrow \downarrow \mathbb{\int}$ Arash Etemadi $\$$, Atkilt Esaiyas Etisso $\$$, Oluchi Ezekannagha $\uparrow$,

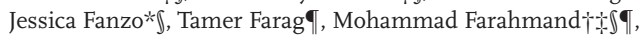
Anwar Faraj†t, Emerito Jose A Faraon -, Mohammad Fareed $†$ \,

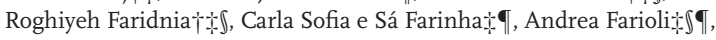

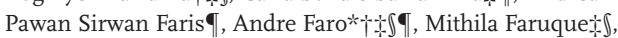

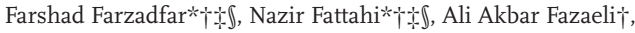

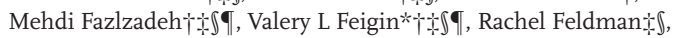

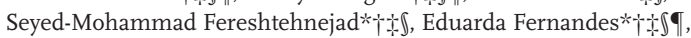

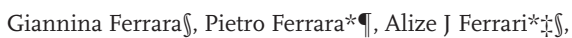

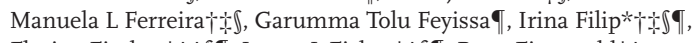

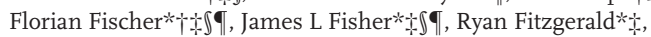

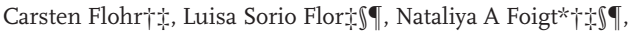

Morenike Oluwatoyin Folayan* $+\uparrow \int$,

Artem Alekseevich Fomenkov*tฐ

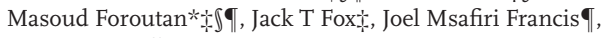

Tahvi D Frank\|, Richard Charles Franklin*』, Marisa Freitas*广t』,

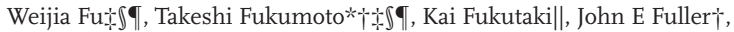

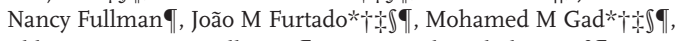

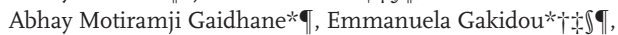

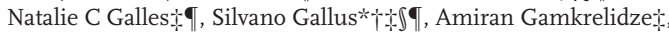

Alberto L Garcia-Basteiro $\$$, William M Gardner* $\$ \llbracket$,

Biniyam Sahiledengle Geberemariyam* $\div$,

Abiyu Mekonnen Gebrehiwot††, Ketema Bizuwork Gebremedhin*†安,

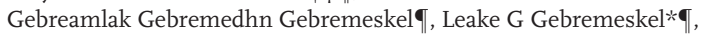
Begashaw Melaku Gebresillassie*,

Assefa Ayalew Ayalew Ayalew Gebreslassieț

Yilma Chisha Dea Geramo*, Abraham Geremewd,

Anna Gershberg Hayoon $\neq \int$, Hailay Abrha Gesesew*ף,

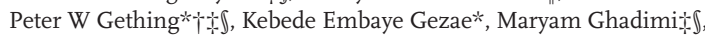

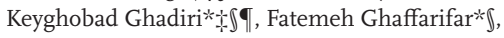

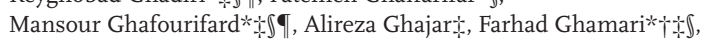

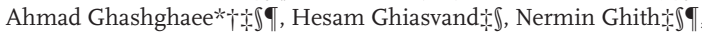

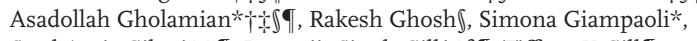

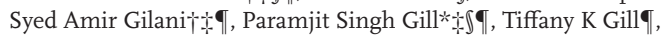

Richard F Gillum*, Ibrahim Abdelmageed Ginawi*,

Themba G Ginindza』 Mojgan Gitimoghaddam $\uparrow+$,

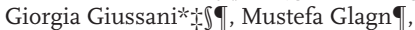

Ekaterina Vladimirovna Glushkova $\uparrow$, Elena V Gnedovskaya*†ฐ

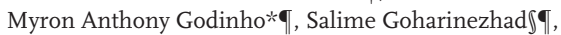

Mahaveer Golechha*, Srinivas Goli $\$$, Ricardo Santiago Gomez†,

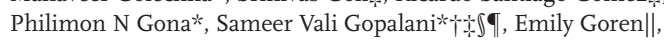

Giuseppe Gorini $₹$ d, Taren M Gorman $\downarrow$, Harrison Chase Gottlich $\nleftarrow$,

Houman Goudarzi $\$$, Amir Hossein Goudarzian $\uparrow$,

Alessandra C Goulart*十+t], Bárbara Niegia Garcia Goulart* $\uparrow$,

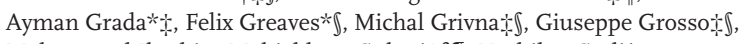
Mohammed Ibrahim Mohialdeen Gubari $₫$ I, Nachiket Gudi*,

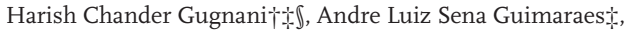

Rafael Alves Guimarães* $\leftarrow$ ฯ

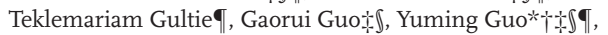

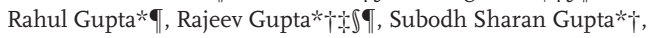
Tarun Gupta』, Juanita A Haagsma*†, Vladimir Hachinski*,

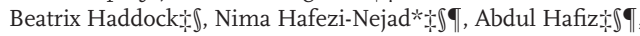

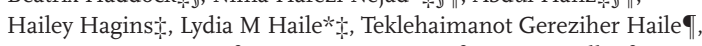
Arvin Haj-Mirzaian* $\uparrow \rrbracket$, Arya Haj-Mirzaian $\uparrow \rrbracket$, Brian J Hall* $+\downarrow$,

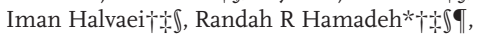

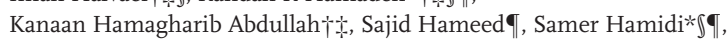
Erin B Hamilton $\leftarrow$, Melanie S Hammer

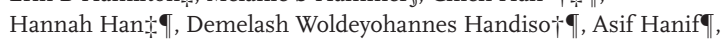

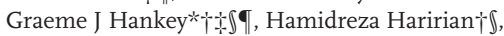

Josep Maria Haro*十丁

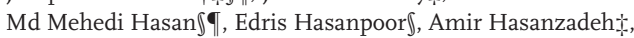

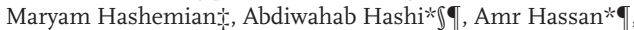

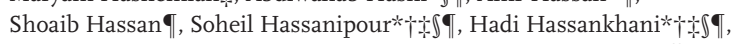

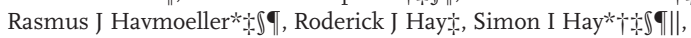

Khezar Hayat* $\leftarrow \mathbb{\Upsilon} \uparrow$, Behzad Heibati $\uparrow$, Behnam Heidari $\$$,

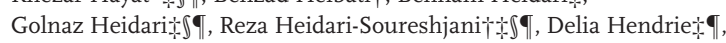

Kiana Henny†, Andualem Henok*, Hannah J Henrikson $\downarrow \mathbb{\downarrow}$,

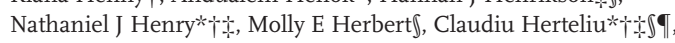

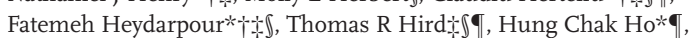

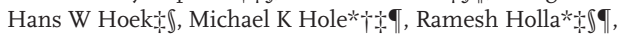

Bruce Hollingsworth $₫$, Praveen Hoogar*十斤

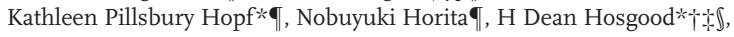

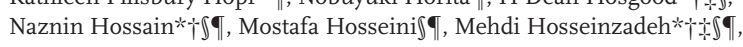

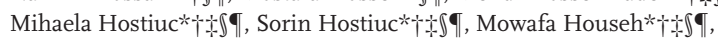

Damian G Hoy*广t, Mohamed Hsairitฐ, Thomas Hsiaol|,

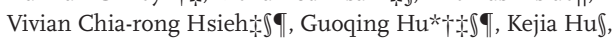

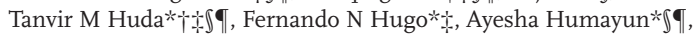

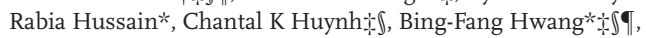

Vincent C Iannuccił』, Ivo Iavicoli* $\$$, Charles Ugochukwu Ibeneme*, 
Segun Emmanuel Ibitoye*十斤

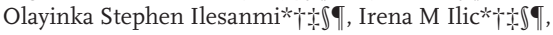

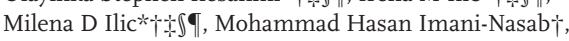

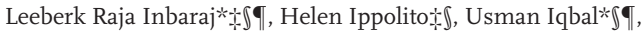

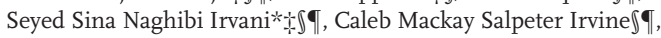
M Mofizul Islam* $+\int$, MdMohaimenul Islam $†$ t Sheikh Mohammed Shariful Islam*t]

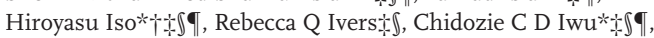

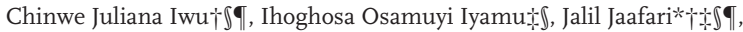
Kathryn H Jacobsen $\uparrow \downarrow$, Farhad Jadidi-Niaragh $\uparrow$, Hussain Jafari $\downarrow \mathbb{\rrbracket}$,

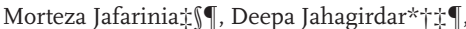

Mohammad Ali Jahani* $*$, Nader Jahanmehr广 + ,

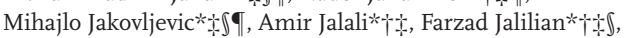

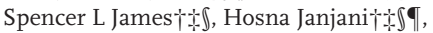

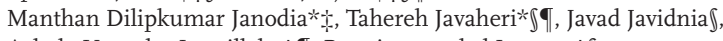
Achala Upendra Jayatilleke $\uparrow$, Panniyammakal Jeemon $\leftarrow$,

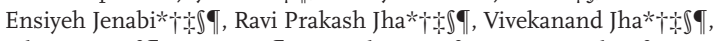

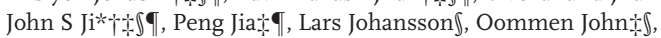
Yetunde O John-Akinolat]

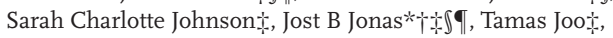

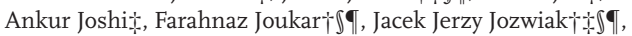

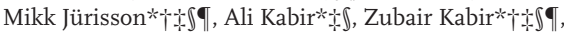

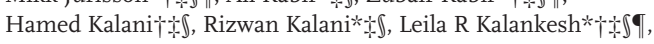

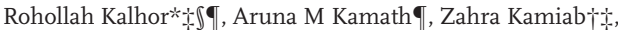
Tanuj Kanchan*十斤

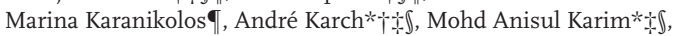
Salah Eddin Karimi*†t, Seyed Asaad Karimi $\uparrow$, Seyed M Karimi $\uparrow$, Ayele Semachew Kasa*, Getachew Mullu Kassał』, Nicholas J Kassebaum*十† , Srinivasa Vittal Katikireddi $\uparrow+\llbracket$, Norito Kawakami $\uparrow+$, Gbenga A Kayode $*+\div \rrbracket$, Ali Kazemi Karyani $* \mathbb{\rrbracket}$,

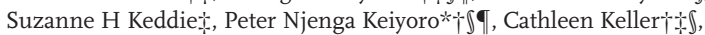
Laura Kemmer』, Parkes J Kendrick』, Maia Kereselidzeł, Yousef Saleh Khader $\uparrow$, Morteza Abdullatif Khafaie

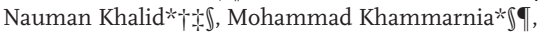

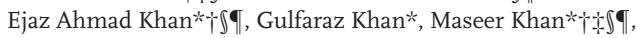

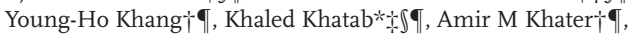

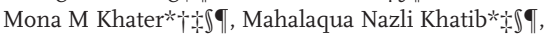
Maryam Khayamzadeh $\$ \llbracket$, Salman Khazaei $\uparrow \rrbracket$, Habibolah Khazaie* ${ }^{*}$, Mohammad Taghi Khodayari†t, Abdullah T Khoja*,

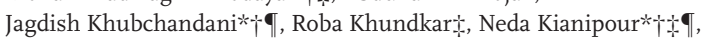

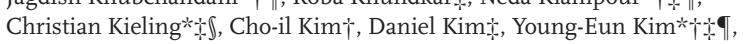

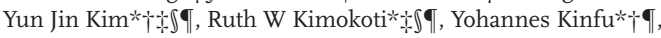

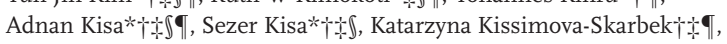

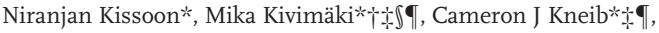

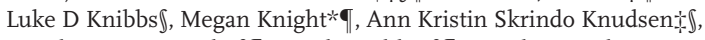
Jonathan M Kocarnik Stefan Kohler $\uparrow$, Tufa Kolola $\ddagger$, Hamidreza Komaki*†, Jacek A Kopec*广一ा, Anna V Korotkova

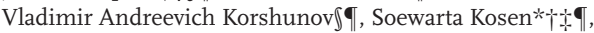

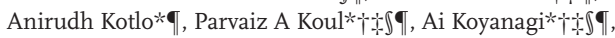
Moritz U G Kraemer』, Michael A Kravchenko $†+$, Kewal Krishan $* \dagger \downarrow \rrbracket$, Kris J Krohn*†tฐף, Hans Kromhout』, Shaji KS*, Barthelemy Kuate Defo $\uparrow+\mathbb{l}$, Burcu Kucuk Bicert, Nuworza Kugbey $\Phi$,

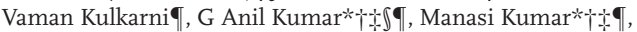

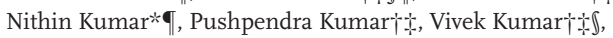

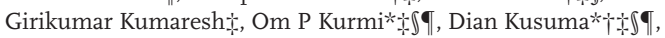
Hmwe Hmwe Kyu*十+

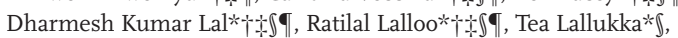

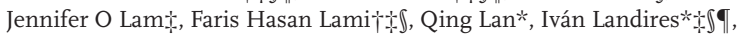

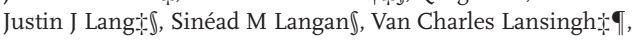
Sonia Lansky†, Heidi Jane Larson*, Samantha Leigh Larson $\uparrow+$,

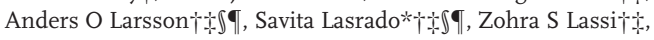

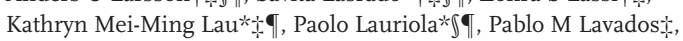

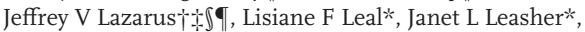

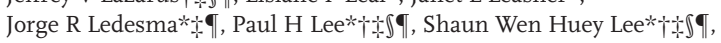

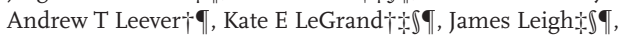

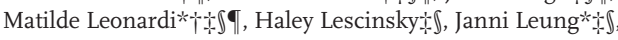

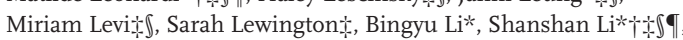

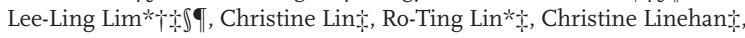

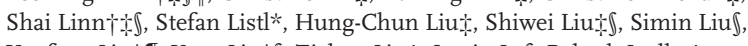

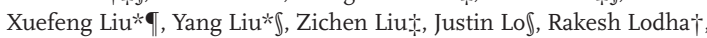

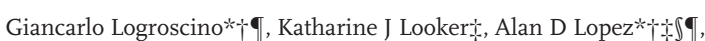

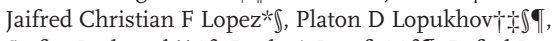

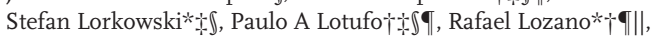

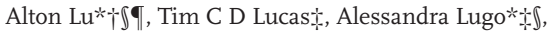

Raimundas Lunevicius*†t, Ronan A Lyons* $*$, Jianing Ma* $\uparrow$,

Daiane Borges Machado*, Jennifer H MacLachlan

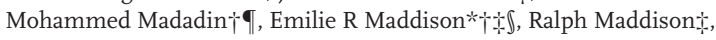

Fabiana Madotto + , Hassan Magdy Abd El Razek†,

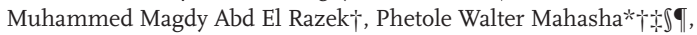

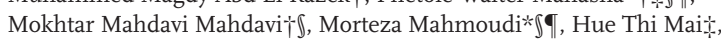

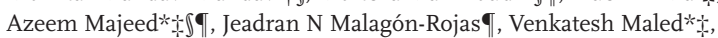

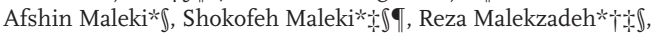

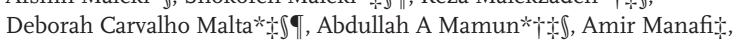

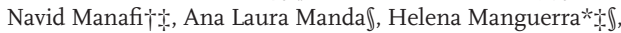

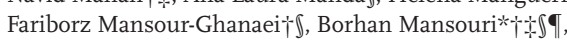

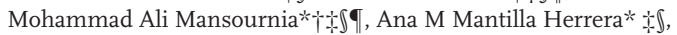

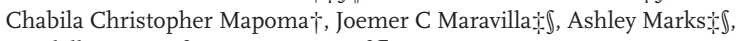
Randall V Martin』, Santi Martini*†ฐף,

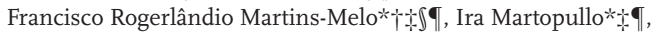

Anthony Masaka†』, Seyedeh Zahra Masoumi*t』, João Massano

Benjamin Ballard Massenburg* $\uparrow$, Claudia I Mastrogiacomo†

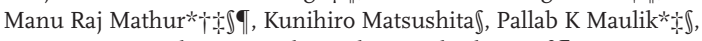

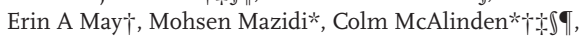

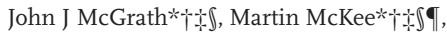

Carlo Eduardo Medina-Solís $\uparrow$, Birhanu Geta Meharie $\uparrow$,

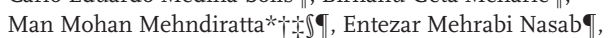

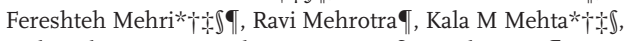

Wahengbam Bigyananda Meitei†t, Teferi Mekonnen $\uparrow$,

Addisu Melese*ף, Peter T N Memiah† $†$, Ziad A Memish*ף,

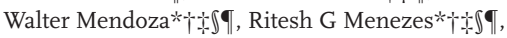

Endalkachew Worku Mengesha*††

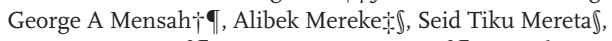

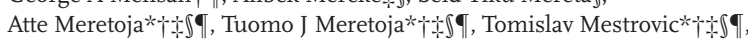

Bartosz Miazgowski*t』』, Tomasz Miazgowski

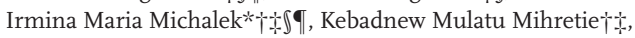

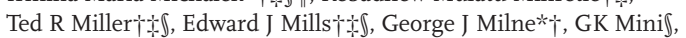

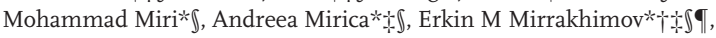

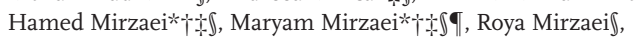

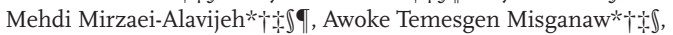

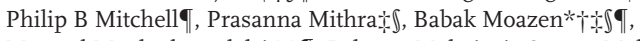

Masoud Moghadaszadeh*† ${ }^{*} \uparrow$, Bahram Mohajer*, Osama Mohamad*,

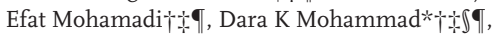

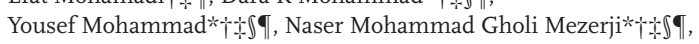

Abolfazl Mohammadbeigi $\uparrow$, Abdollah Mohammadian-Hafshejani $* \dagger+\downarrow$, Noushin Mohammadifard $\leftarrow \mathbb{\int}$, Reza Mohammadpourhodki $† \uparrow$,

Ammas Siraj Mohammed』, Hussen Mohammed $\uparrow \rrbracket$,

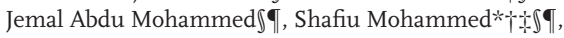

Farnam Mohebi ${ }^{*} \uparrow$, Mohammad A Mohseni Bandpei $\uparrow$, Amin Mokari $\uparrow$,

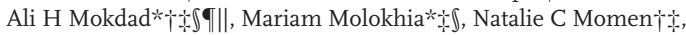

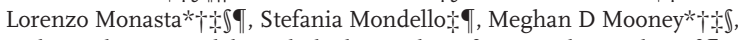

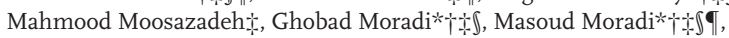

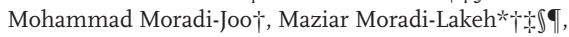

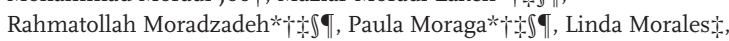

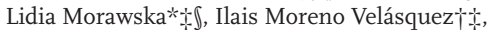

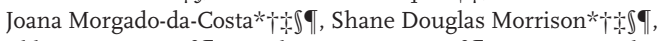

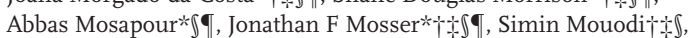

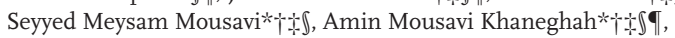

Ulrich Otto Mueller*†t

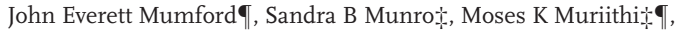

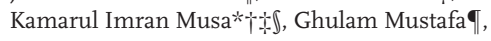

Saravanan Muthupandian $\uparrow+\$$, Behnam Nabavizadeh $\uparrow$,

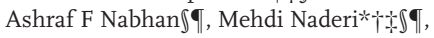

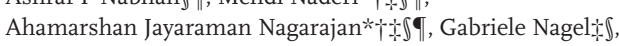

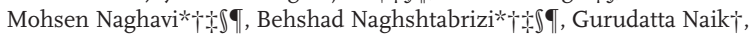
Mukhammad David Naimzada*』 , Sanjeev Nair Luigi Naldi*, Vishnu Nandakumar||, Anita K Nandił, Vinay Nangia†⿰

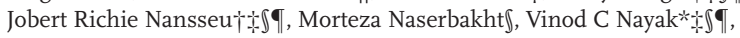
Javad Nazari*ף, Rawlance Ndejo* $*$, Duduzile Edith Ndwandwe $†$,

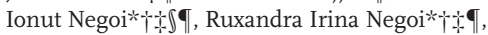

Henok Biresaw Netsere $*$, Subas Neupane $\boldsymbol{q}$, Kiirithio N Ngari $\boldsymbol{q}$,

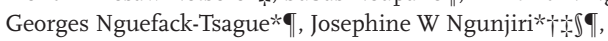




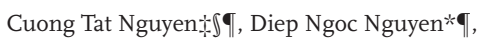

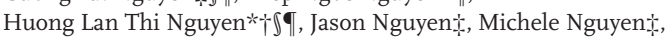
Ming Nguyen $\leftarrow$, Trang Huyen Nguyen $\dagger$, Emma Nichols ${ }^{*}+$, Dabere Nigatuł, Yeshambel T Nigatu* $* t$,

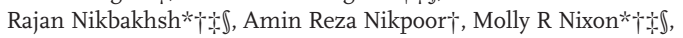

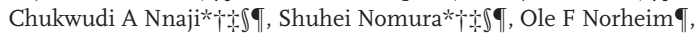
Bo Norrvingt: Jean Jacques Noubiap*广t] Christoph Nowak ${ }^{*}+\mathrm{f}$ II, Elaine Okanyene Nsoesie*,

Virginia Nunez-Samudio*t

Christopher M Odell $\uparrow \downarrow$

Onome Bright Oghenetega $* \boldsymbol{\Phi}$, In-Hwan Oh†t/

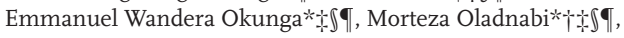

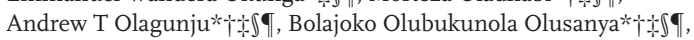

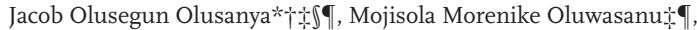

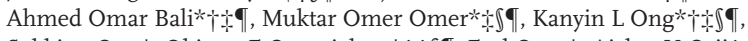

Sokking Ong*, Obinna E Onwujekwe*十斤

Heather M Orpanat: Doris V Ortega-Altamirano 9 , Alberto Ortiz

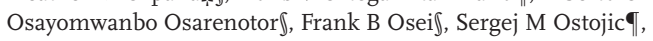

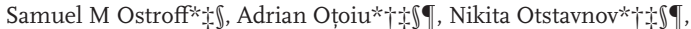

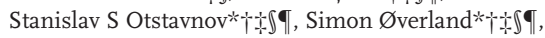

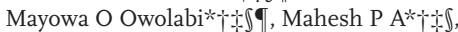

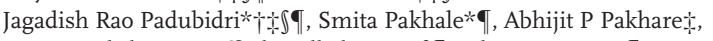

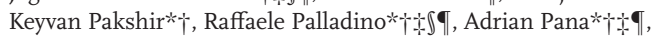

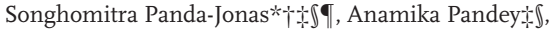

Helena Ullyartha Pangaribuan $\uparrow$, Eun-Kee Park

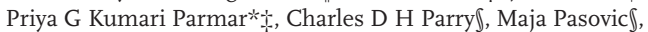

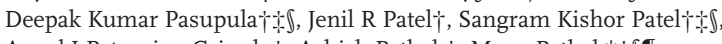

Angel J Paternina-Caicedo

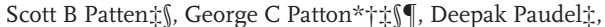

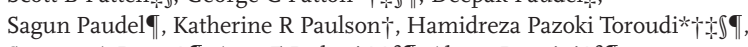

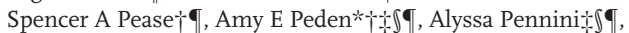

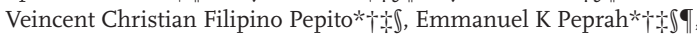

Alexandre Pereira $* \dagger$, David M Pereira ${ }^{*}+t$, Jeevan Pereira $* \boldsymbol{\Phi}$,

Norberto Perico*广t, Julia Moreira Pescarini*, Konrad Pesudovs ,

Hai Quang Pham* $*$, Michael R Phillips* $*$, Cristiano Piccinelli $* \mathbb{\int}$,

Maxwell Piercel|, David M Pigott $\uparrow \mp$, Thomas Pilgrim $†-$,

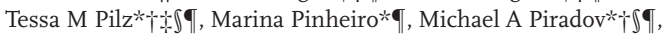

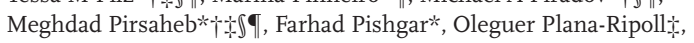

Dietrich Plasst: J, Martin Pletcher||, Khem Narayan Pokhrel†

Roman V Polibin

Constance Dimity Pond $* \mathbb{\int}$, Maarten J Postma*广tr

Faheem Hyder Pottoo*, Hadi Pourjafar*广t

Farshad Pourmalek*t Reza Pourmirza Kalhorit,

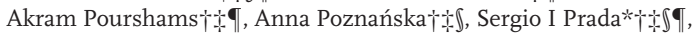

Sanjay Prakash $\uparrow$, V Prakash $\hbar$ J , Narayan Prasad $\star$, Liliana Preotescu $\dagger$,

Dimas Ria Angga Pribadi $*+\int \boldsymbol{\Psi}$, Elisabetta Pupillo*t]

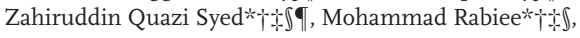

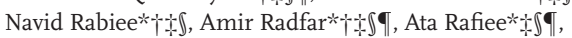

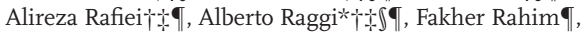

Afarin Rahimi-Movaghart, Mohammad Hifz Ur Rahman*,

Muhammad Aziz Rahman*广大

Fatemeh Rajatiț Ivo Rakovac』, Pradhum Ram $\uparrow \rrbracket$,

Kiana Ramezanzadeh* $*$, Saleem Muhammad Rana $\boldsymbol{q}$,

Chhabi Lal Ranabhat*广†

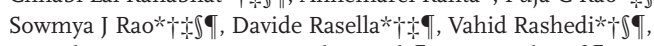

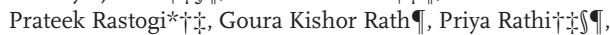

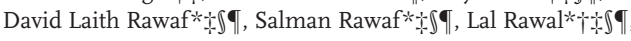

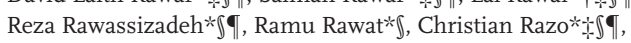

Sofia Boston Redford $\$$ l, Lemma Demissie Regassa*,

Robert C Reiner Jrt: Nickolas Reinig

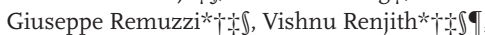

Andre M N Renzaho*十t

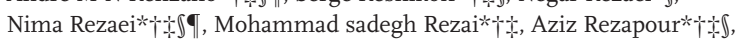

Phoebe-Anne Rhinehart*t], Seyed Mohammad Riahi*t]

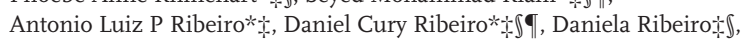

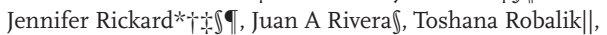

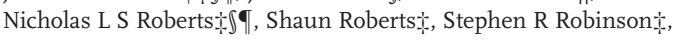

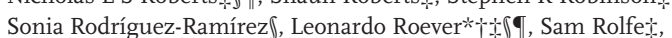

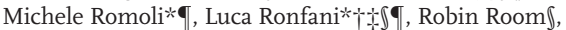

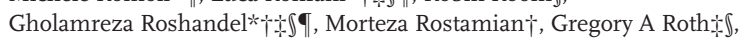

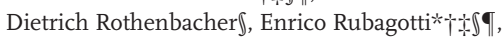

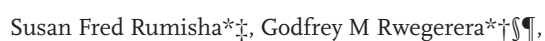

Seyedmohammad Saadatagah*, Siamak Sabour*十t

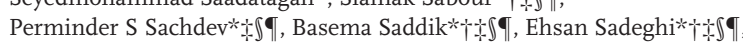

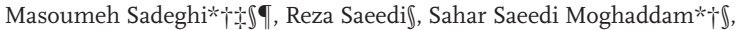

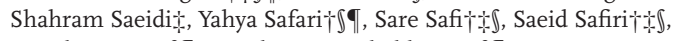

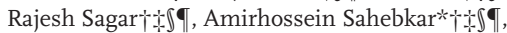

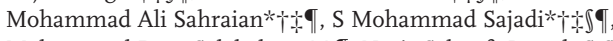

Mohammad Reza Salahshoor †

Payman Salamati†

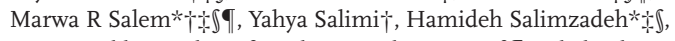

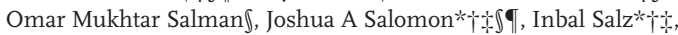

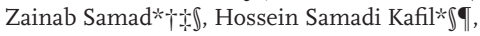

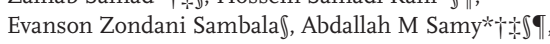

Juan Sanabria*†

Damian Francesco Santomauro*t], Itamar S Santost],

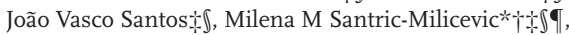

Sivan Yegnanarayana Iyer Saraswathy*广†

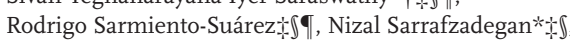

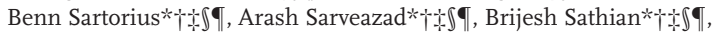

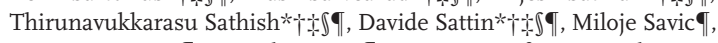

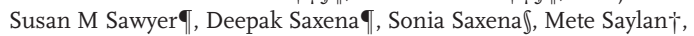

Alyssa N Sbarrał

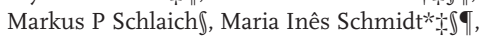

Aletta Elisabeth Schutte*t]

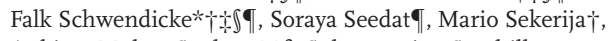

Anbissa Muleta Senbeta†t Subramanian Senthilkumaran†t:

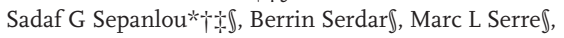

Edson Serván-Mori† $†$, Feng Sha*đ, Mahsima Shabani $†$,

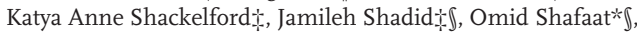

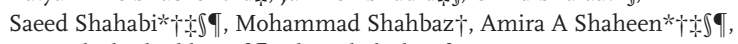

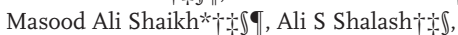

Mehran Shams-Beyranvand ${ }^{*} \dagger \uparrow$, MohammadBagher Shamsi $\uparrow$,

Morteza Shamsizadeh*广†

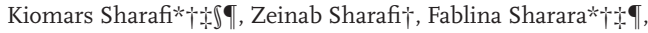

Hamid Sharifiף, Rajesh Sharma†', David H Shaw $\uparrow$,

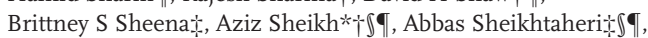

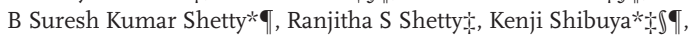

Kevin David Shield , Wondimeneh Shibabaw Shiferaw:

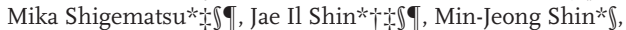

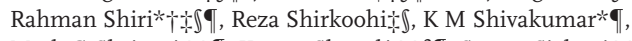

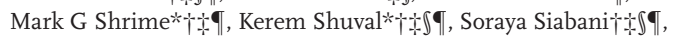

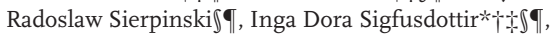

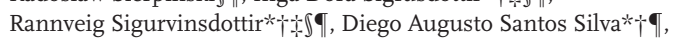

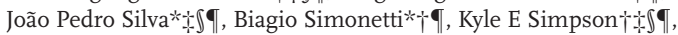

Ambrish Singh $*+$, Jasvinder A Singh $*+\uparrow \Upsilon$, Pushpendra Singh $* \llbracket$,

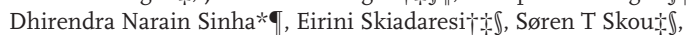

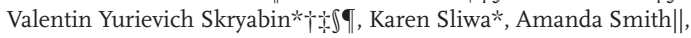

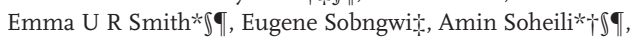

Anton Sokhan†t, Shahin Soltani ${ }^{*}+\boldsymbol{\Phi}$, Oluwaseyi Dolapo Somefun*,

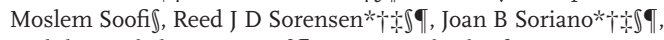

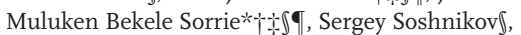

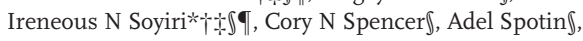

Emma Elizabeth Spurlock $\uparrow$, Chandrashekhar T Sreeramareddy ${ }^{*} \dagger+\emptyset$,

Vinay Srinivasan』, Kam Sripada*, Jeffrey D Stanaway*t]

Benjamin A Starkł, Nicholas Steelף, Simona Cătălina Ștefan*ț,

Caroline Stein

Timothy J Steinert; Krista M Steuben\|, Leo Stockfelt $\uparrow \rrbracket$,

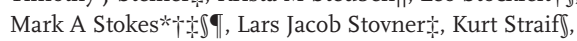

Saverio Stranges*, Jacob L Stubbs $† \neq \mathbb{f}$, Parminder S Suchdev*,

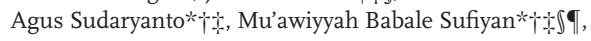

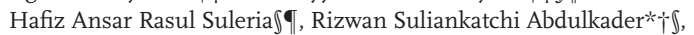

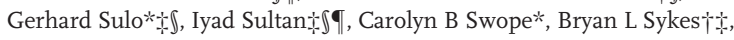

Dillon O Sylte*ł, Miklós Szócskałt, Lukasz Szumowskiף,

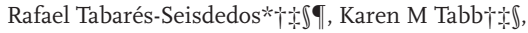

Takahiro Tabuchi $*$ I, Santosh Kumar Tadakamadla*十斤

Biruk Wogayehu Taddele* $\approx$, Degena Bahrey Tadesse $\uparrow$,

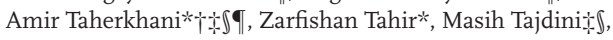

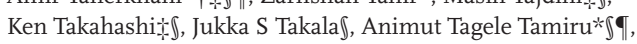

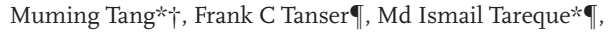

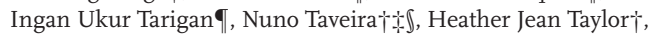

Whitney L Teagle†t, Hirut Teame‡, Fabrizio Tediosi $\uparrow$, 


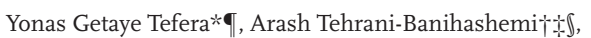

Berhane Fseha Teklehaimanot†t, Freweini Gebrearegay Tela*, Mohamad-Hani Temsah*ø, Sonyah Terrason* $\%$,

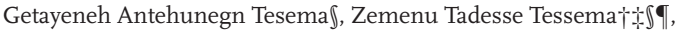
Bhaskar Thakur* , Kavumpurathu Raman Thankappan*†+, Rekha Thapar*, Nihal Thomas* $\dagger$, Azalea M Thomson

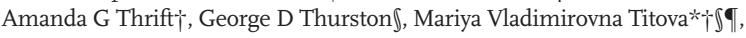
Boikhutso Tlou*, Hamid Reza Tohidinik†t

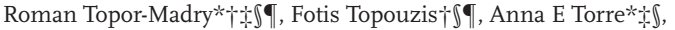

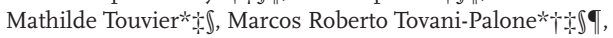

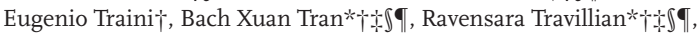
Sergi Trias-Llimósł, Christopher E Troegerł

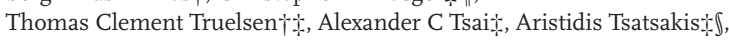

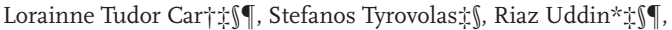
Irfan Ullah*ף, Saif Ullah Eduardo A Undurraga $\$$, Bhaskaran Unnikrishnan*†⿰\zh9,

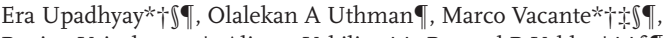

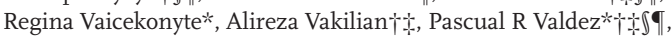
Alessandro Valli ${ }^{\dagger} \uparrow$, Aaron van Donkelaar】, Constantine Vardavas $\dagger$, Santosh Varughese $\downarrow$, Tommi Juhani Vasankari*广就,

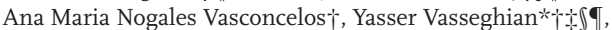

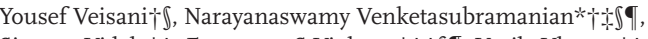

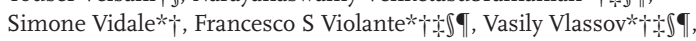

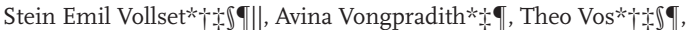
Giang Thu Vu*, Isidora S Vujcic $\dagger$, Ana Vukovic $†+$, Rade Vukovic $† \downarrow$, Feleke Gebremeskel W/hawariat*, Yasir Waheed*广t行, Mitchell Taylor Wallin $\uparrow \mathbb{\rrbracket}$, Magdalene K Walters $\dagger+\boldsymbol{T}$ Richard G Wamai*ף, Fang Wang*, Haidong Wang*† $\uparrow$, Hongbo Wang $\uparrow$, Jiayu Wang*t, Yafeng Wangt: Yanzhong Wang*,

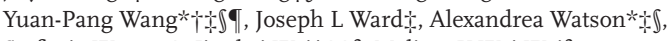

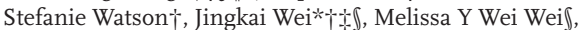

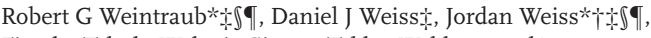
Fissaha Tekulu Welay*, Girmay Teklay Weldesamuel $\uparrow$,

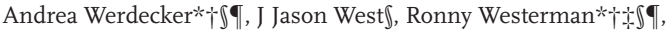
Joanna L Whisnant Taweewat Wiangkham*†t, Nuwan Darshana Wickramasinghe* Kirsten E Wiens†t Shadrach Wilson $\downarrow$, Charles Shey Wiysonge $\uparrow$, Bogdan Wojtyniak* $\uparrow$, Gebremariam Woldu 9 , Charles D A Wolfe*广t Temesgen Gebeyehu Wondmeneh*, Adam Belay Wondmieneh $\uparrow$,

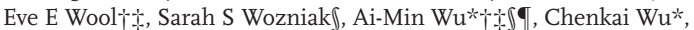

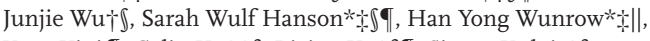

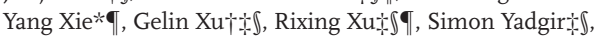

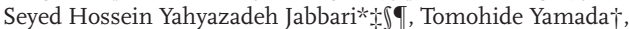

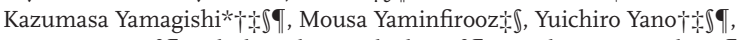

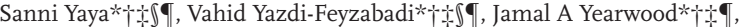
Tomas Y Yeheyis*†t

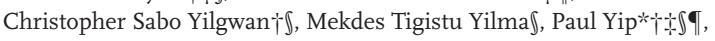

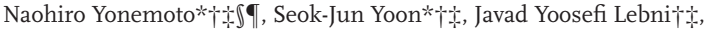

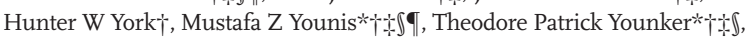
Bahman Yousefi『, Zabihollah Yousefi $†$ I, Mahmoud Yousefifard $t$,

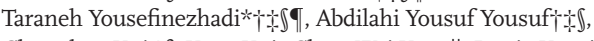
Chuanhua Yu* $*$, Yong Yu*, Chun-Wei Yuan||, Deniz Yuce*,

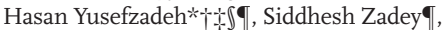
Telma Zahirian Moghadam $\uparrow+\downarrow$, Syed Saoud Zaidi $*$, Leila Zaki $\uparrow$, Josefina Zakzuk†, Sojib Bin Zaman*††, Mohammad Zamani†场,

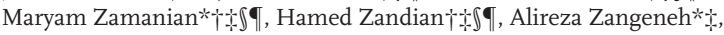
Hadi Zarafshan*, Mikhail Sergeevich Zastrozhin* $\leftarrow$ 『,

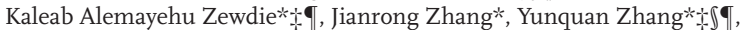

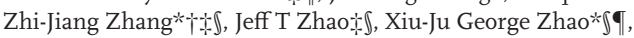

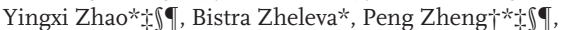

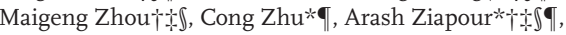

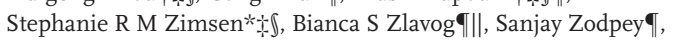

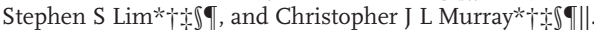
*GBD 2019 Overview Collaborators. †GBD 2019 Demographics Collaborators. $†$ GBD 2019 Diseases and Injuries Collaborators. 『GBD 2019 Risk Factors Collaborators. TGBD 2019 UHC Collaborators. ||GBD 2019 Forecasting Collaborators.

\section{Affiliations}

Department of Juridical and Economic Studies (C Abbafati PhD), La Sapienza University, Rome, Italy; Centre for Global Mental Health
(CGMH) (D B Machado PhD), Department of Global Health and Development (B Cislaghi PhD, O M Salman MSc), Department of Health Services Research and Policy (M Karanikolos PhD, Prof M McKee DSc), Department of Infectious Disease Epidemiology (K M Abbas PhD, O J Brady PhD, Prof H J Larson PhD), Department of Non-communicable Disease Epidemiology (S Trias-Llimós PhD), Department of Public Health, Environments, and Society (Prof S Cummins PhD), European Observatory on Health Systems and Policies (M Karanikolos PhD), Faculty of Epidemiology and Population Health (Prof S M Langan PhD), Faculty of Infectious and Tropical Diseases (Prof B Sartorius PhD), MSc Epidemiology Programme (A Hafiz PhD), London School of Hygiene \& Tropical Medicine, London, UK; Autism Spectrum Disorders Research Center (E Jenabi PhD), Department of Biostatistics (N Mohammad Gholi Mezerji MSc), Department of Endocrinology (S Borzouei MD), Department of Environmental Health Engineering (G Azarian PhD), Department of Epidemiology (S Khazaei PhD), Department of Internal Medicine (M Abbasi PhD), Department of Microbiology (B Asghari PhD), Department of Midwifery (S Masoumi PhD), Neurophysiology Research Center (H Komaki MD), Research Center for Molecular Medicine (A Taherkhani PhD), Hamadan University of Medical Sciences, Hamadan, Iran (M Adabi PhD); Clinical Research Development Unit (M Abbasifard MD, G Bazmandegan PhD, Z Kamiab MD), Department of Internal Medicine (M Abbasifard MD, G Bazmandegan PhD), Department of Neurology (A Vakilian MD), Department of Family Medicine (Z Kamiab MD), Non-communicable Diseases Research Center (A Vakilian MD), Social Determinants of Health Research Center (M Anjomshoa PhD), Rafsanjan University of Medical Sciences, Rafsanjan, Iran; Department of Community Nutrition (A Mokari PhD), Department of Epidemiology (S Sabour PhD, M Shahbaz MSc), Department of Health, Safety, and Environment (HSE) (R Saeedi PhD), Department of Ophthalmology (H Ahmadieh MD), Injury Prevention and Safety Promotion Research Center (T Yousefinezhadi PhD), Obesity Research Center (A Haj-Mirzaian MD, R Nikbakhsh MD), Ophthalmic Epidemiology Research Center (S Safi PhD), Ophthalmic Research Center (H Ahmadieh MD, S Safi PhD), Prevention of Metabolic Disorders Research Center (S Asgari MSc), Research Institute for Endocrine Sciences (S N Irvani MD), Safety Promotion and Injury Prevention Research Center (N Jahanmehr PhD), School of Management and Medical Education (N Jahanmehr PhD), School of Pharmacy (K Ramezanzadeh PharmD), Social Determinants of Health Research Center (M Abbasi-Kangevari MD), Shahid Beheshti University of Medical Sciences, Tehran, Iran (M Khayamzadeh MD); Advanced Diagnostic and Interventional Radiology Research Center (H Abbastabar PhD), Cancer Biology Research Center (R Shirkoohi PhD), Cancer Research Institute (R Shirkoohi PhD), Department of Environmental Health Engineering (M Fazlzadeh PhD, $\mathrm{H}$ Janjani PhD), Department of Epidemiology and Biostatistics (Prof M Hosseini PhD, M Mansournia PhD, H Tohidinik PhD), Department of Health Policy, Management, and Economics (A Bakhtiari PhD, A Fazaeli PhD, S Mousavi PhD), Department of Microbiology (A Hasanzadeh PhD), Department of Pharmacology (A Haj-Mirzaian MD), Department of Urology (B Nabavizadeh MD), Digestive Diseases Research Center (Prof R Malekzadeh MD), Digestive Diseases Research Institute (M Hashemian PhD,

Prof A Pourshams MD, H Salimzadeh PhD, S G Sepanlou MD), Endocrinology and Metabolism Research Center (M Afarideh MD, Prof A Esteghamati MD, S Esteghamati MD, A Ghajar MD, B Heidari MD, N Rezaei PhD), Health Equity Research Center (E Mohamadi PhD), Iranian National Center for Addiction Studies (INCAS) (Prof A Rahimi-Movaghar MD), Liver and Pancreaticobilliary Disease Research Center (H Ebrahimi MD), Metabolomics and Genomics Research Center (F Rahim PhD), Multiple Sclerosis Research Center (S Eskandarieh PhD, Prof M Sahraian MD), National Institute of Health Research (NIHR) (F Mohebi MD), Non-communicable Diseases Research Center (A Aminorroaya MD, H Ebrahimi MD

Prof F Farzadfar DSc, B Mohajer MD, F Mohebi MD, F Pishgar MD, N Rezaei PhD, S Saeedi Moghaddam MSc, M Shabani MD), Pediatric Chronic Kidney Disease Research Center (Prof M Hosseini PhD), Psychiatry and Psychology Research Center (H Zarafshan PhD), Research Center for Immunodeficiencies (H Abolhassani PhD, 
Prof N Rezaei PhD), School of Medicine (N Hafezi-Nejad MD), School of Nursing and Midwifery (R Heidari-Soureshjani MSc), School of Pharmacy (Prof M Abdollahi PhD), School of Public Health (M Farahmand PhD), Sina Trauma and Surgery Research Center (Prof P Salamati MD), Tehran Heart Center (A Aminorroaya MD, E Mehrabi Nasab MD, M Tajdini MD), The Institute of Pharmaceutical Sciences (TIPS) (Prof M Abdollahi PhD), Uro-oncology Research Center (F Pishgar MD), Urology Research Center (Prof S Aghamir PhD), Water Quality Research Center (R Mirzaei PhD), Tehran University of Medical Sciences, Tehran, Iran (Z Dibaji Forooshani PhD); Department of Medical Parasitology (M M Khater MD), Department of Neurology (Prof F Abd-Allah MD, Prof A Abdelalim MD, A Abualhasan MD, S I El-Jaafary MD, A Hassan MD), Endemic Medicine and Hepatogastroentrology Department (A Elsharkawy MD), National Hepatology and Tropical Medicine Research Institute (A M Khater MD), Neurophysiology Department (Prof H R Elhabashy MD), Public Health and Community Medicine Department (M R R Salem MD), Urology Department (Prof H Salem MD), Cairo University, Cairo, Egypt; Cardiac Rehabilitation Research Center (Prof M Sadeghi MD), Department of Immunology (M Jafarinia MSc), Health Management and Economics Reasearch Center (M Amini-Rarani PhD), Isfahan Cardiovascular Research Institute (N Mohammadifard PhD, Prof N Sarrafzadegan MD), Neuroscience Research Center (I Abdollahpour PhD), Isfahan University of Medical Sciences, Isfahan, Iran; Colorectal Research Center (A Sarveazad PhD), Comprehensive Research Laboratory (R Mirzaei PhD), Department of Community and Family Medicine (A Tehrani-Banihashemi PhD), Department of Health Education and Promotion (J Yoosefi Lebni MA), Department of Ophthalmology (N Manafi MD), Department of Physiology (H Pazoki Toroudi PhD), Faculty of Allied Medicine (F Dorostkar PhD), Health Economics Department (V Alipour PhD), Health Information Management (A Sheikhtaheri PhD), Health Management and Economics Research Center (V Alipour PhD, J Arabloo PhD, S Azari PhD,

A Ghashghaee BSc, A Rezapour PhD), Mental Health Research Center (M Naserbakht MD), Minimally Invasive Surgery Research Center (A Kabir MD), Nutrition Health Research Center (F Mehri PhD), Physiology Research Center (H Pazoki Toroudi PhD, M Yousefifard PhD), Preventive Medicine and Public Health Research Center (M Asadi-Aliabadi MSc, E Babaee PhD, B Eshrati PhD, S Goharinezhad PhD, M Moradi-Lakeh MD, M Naserbakht MD, A Tehrani-Banihashemi PhD), School of Medicine (P Abedi MD), Student Research Committee (A Ghashghaee BSc), Tehran Institute of Psychiatry (V Rashedi PhD), Iran University of Medical Sciences, Tehran, Iran; Brigham and Women's Hospital (V Kumar MD), Cardiology Department (I Y Elgendy MD), Center for Primary Care (S Basu PhD), Department of Biostatistics (J Park MSc), Department of Genetics (A Pereira PhD), Department of Global Health and Population (Prof O F Norheim PhD), Department of Global Health and Social Medicine (A W Eagan MSW), Department of Nutrition (L E Cahill PhD), Division of General Internal Medicine (Prof A Sheikh MD), Harvard Medical School (A I Abushouk MD, M U G Kraemer PhD),

Massachusetts Veterans Epidemiology Research and Information Center (MAVERIC) \& CSP Coordinating Center (B Thakur PhD), Neuroendocrine (P Abedi MD), T.H. Chan School of Public Health (Prof T W Bärnighausen MD), Harvard University, Boston, MA, USA (M G Shrime MD); Department of Orthopaedic Surgery (A Abedi MD), Keck School of Medicine (C P Doshi BA), University of Southern California, Los Angeles, CA, USA; Biostatistics Department (K H Abegaz MSc), Near East University, Nicosia, Cyprus; Biostatistics and Health Informatics (K H Abegaz MSc), Department of Public Health (B S Geberemariyam MPH), Madda Walabu University, Bale Robe, Ethiopia; Karolinska University Hospital, Huddinge, Sweden (H Abolhassani PhD); Department of Nursing (Y A Aynalem MSc, W S Shiferaw MSc), Department of Public Health (A E Abosetugn MPH), Debre Berhan University, Debre Berhan, Ethiopia; Department of Cardiology (Prof V Aboyans MD), Dupuytren University Hospital, Limoges, France; University of Limoges, Limoges, France (Prof V Aboyans MD); Ophthalmology Department (N Manafi MD), Section of Allergy and Clinical Immunology (E M Abrams MD), University of Manitoba, Winnipeg, MB, Canada; Department of Pediatrics (E M Abrams MD, M Gitimoghaddam MD,
Prof N Kissoon MD), Department of Psychiatry (J L Stubbs BKin), School of Population and Public Health (Prof M Brauer DSc, I O Iyamu MD, J A Kopec PhD, F Pourmalek PhD, Prof N Sarrafzadegan MD), University of British Columbia, Vancouver, BC, Canada; Centre of Telehealth (Prof A P Ribeiro MD), Department of Internal Medicine (Prof A P Ribeiro MD), Department of Maternal and Child Nursing and Public Health (Prof D C Malta PhD), Department of Oral Surgery and Pathology (Prof R S Gomez PhD), Department of Pediatric Dentistry (Prof L G Abreu PhD), Federal University of Minas Gerais, Belo Horizonte, Brazil; Department of Research (M R M Abrigo PhD), Philippine Institute for Development Studies, Quezon City, Philippines; College of Medicine (A M Almulhim MBBS), Environmental Health Department (S M A Dahlawi PhD), Pharmacology Department (F H Pottoo PhD), Forensic Medicine Division (Prof R G Menezes MD), Health Information Management and Technology Department (T M Alanzi PhD, A K Alumran PhD), Internal Medicine Department (A K Abu Haimed MBBS), Pathology Department (M Madadin MD), Imam Abdulrahman Bin Faisal University, Dammam, Saudi Arabia (F M Alanezi PhD); Department of Clinical Sciences (E Abu-Gharbieh PhD), Department of Family and Community Medicine (B Saddik PhD), University of Sharjah, Sharjah,

United Arab Emirates; Department of Healthcare Policy and Research (Prof L J Abu-Raddad PhD), Weill Cornell Medical College in Qatar, Doha, Qatar; Department of Entomology (A M Samy PhD), Department of Medicine (A I Abushouk MD), Department of Neurology (Prof N El Nahas MD, Prof A S Shalash PhD), Department of Obstetrics and Gynecology (Prof A F Nabhan PhD), Ain Shams University, Cairo, Egypt; Department of Anesthesiology \& Pain Medicine

(A M Kamath MD, N J Kassebaum MD), Department of Applied Math (A Y Aravkin PhD), Department of Global Health (S Kochhar MD, R J D Sorensen MPH), Department of Health Metrics Sciences, School of Medicine (A Afshin MD, A Y Aravkin PhD, K Burkart PhD, E A Cromwell PhD, Prof L Dandona MD, Prof S D Dharmaratne MD, Prof E Gakidou PhD, Prof S I Hay FMedSci, N J Kassebaum MD, H H Kyu PhD, Prof A D Lopez PhD, Prof R Lozano MD, A T Misganaw PhD, Prof A H Mokdad PhD, Prof M Naghavi MD, D M Pigott PhD, R C Reiner Jr PhD, G A Roth MD, Prof B Sartorius PhD, J D Stanaway PhD, Prof S Vollset DrPH, Prof T Vos PhD, H Wang PhD, Prof S S Lim PhD,

Prof C J L Murray PhD), Department of Neurology (R Kalani MD), Division of Allergy and Infectious Diseases (K S Ikuta MD), Division of Cardiology (G A Roth MD), Division of Plastic Surgery (D Y Cho MD, C J Kneib MD), Division of Plastic \& Reconstructive Surgery (C S Crowe MD, B B Massenburg MD, S D Morrison MD), Institute for Health Metrics and Evaluation (A Acebedo MPH, J D Adelson PhD, A Afshin MD, K M Agesa BA, T Alam MPH, S B Albertson BS, J A Anderson BS, C M Antony MA, A Y Aravkin PhD, C Ashbaugh MA, M Assmus BA, G Azhar PhD, S Balassyano BS, M S Bannick BS, C M Barthelemy MPH, R G Bender BS, F B Bennitt BA, G J Bertolacci BS, M H Biehl MPH, C Bisignano MPH, A S Boon-Dooley BA, Prof M Brauer DSc, P S Briant BS, D Bryazka BA, B R Bumgarner MBA, K Burkart PhD, C S Callender BS, J Cao MS, C D Castle BS, E Castro MS, K Causey BS, K M Cercy BS, J Chalek BS, F J Charlson PhD, A J Cohen DSc, H Comfort BS, K Compton BS, E A Cromwell PhD, A J Croneberger BSc, J A Cruz BS, M Cunningham MSc, Prof L Dandona MD, Prof R Dandona PhD, W J Dangel MEd, F E Dean BA, N K DeCleene BS, A Deen MPH, Prof L Degenhardt PhD, Prof S D Dharmaratne MD, Z V Dingels BS, I N Dippenaar BA, M A Dirac MD, A J Dolgert PhD, S Emmons-Bell BA, K Estep MPA, T Farag PhD, Prof V L Feigin PhD, R Feldman BS, G Ferrara BA, A J Ferrari PhD, R Fitzgerald BS, L M Force MD, J T Fox BS, T D Frank BS, W Fu MSc, K Fukutaki BA, J E Fuller MLIS, N Fullman MPH, Prof E Gakidou PhD, N C Galles BS, W M Gardner AB, A Gershberg Hayoon MSc, E Goren PhD, T M Gorman BS, H C Gottlich BS, G Guo MPH, B Haddock BA, H Hagins MSPH, L M Haile BA, E B Hamilton MPH, C Han BA, H Han MSc, J D Harvey BS, Prof S I Hay FMedSci, K Henny BA, H J Henrikson HSD, N J Henry BS, M E Herbert MSc, T Hsiao BS, C K Huynh BA, V C Iannucci BA, K S Ikuta MD, H Ippolito BA, C M S Irvine BS, H Jafari BA, D Jahagirdar PhD, S L James MD C O Johnson PhD, S C Johnson MS, N J Kassebaum MD, C Keller MPH, 
L Kemmer PhD, P J Kendrick BS, M Knight BS, J M Kocarnik PhD, K J Krohn MPH, H H Kyu PhD, Prof H J Larson PhD, S L Larson BS, K M Lau BS, J R Ledesma BA, A T Leever BS, K E LeGrand MPH, H Lescinsky BA, C Lin BS, H Liu MS, Z Liu BA, J Lo BA, Prof A D Lopez PhD, Prof R Lozano MD, A Lu MSc, J Ma MS, E R Maddison BS, H Manguerra BS, A Marks MA, I Martopullo MPH, C I Mastrogiacomo BS, E A May BIE, A T Misganaw PhD, Prof A H Mokdad PhD, M D Mooney BS, J F Mosser MD, E C Mullany BA, J Mumford BA, S B Munro PhD, Prof M Naghavi MD, V Nandakumar MS, J Nguyen BS, M Nguyen BA, E Nichols BA, M R Nixon PhD, C M Odell MPP, K L Ong PhD, A U Orji BA, S M Ostroff PhD, M Pasovic MA, K R Paulson BS, S A Pease BS, A Pennini MSc, M Pierce BS, D M Pigott PhD, T M Pilz BA, M Pletcher BS, P C Rao MPH, C Razo PhD, S B Redford MPH, R C Reiner Jr PhD, N Reinig BS, M B Reitsma BS, P Rhinehart BA, T Robalik BSc, S Roberts MSc, N L S Roberts BS, S Rolfe BA, G A Roth MD, A N Sbarra MPH, L E Schaeffer MS, K A Shackelford BA, J Shadid BSc, F Sharara MS, D H Shaw BA, B S Sheena BS, K E Simpson BS, A Smith MPA, R J D Sorensen MPH, C N Spencer BA, E E Spurlock BA, J D Stanaway PhD, B A Stark MA, C Steiner MPH, K M Steuben MS, D O Sylte BA, M Tang MS, H J Taylor BA, S Terrason MA, A M Thomson BA, A E Torre BS, R Travillian PhD, C E Troeger MPH, Prof S Vollset DrPH, A Vongpradith BA, Prof T Vos PhD, M K Walters BS, H Wang PhD, J Wang MPH, A Watson BA, S Watson MS, J L Whisnant MPH,

Prof H A Whiteford PhD, K E Wiens PhD, L B Wilner MPH, S Wilson BS, E E Wool MPH, S S Wozniak BA, J Wu BA, S Wulf Hanson PhD, H Wunrow BSc, R Xu BS, S Yadgir MPH, J A Yearwood BS, H W York BA, C Yuan PhD, J T Zhao BA, P Zheng PhD, S R M Zimsen MA, B S Zlavog BS, Prof S S Lim PhD, Prof C J L Murray PhD), University of Washington, Seattle, WA, USA (A Y Chang DSc, Prof E Oren PhD); Department of Epidemiology and Preventive Medicine (Prof R Buchbinder PhD, K L Chin PhD,

Prof Y Guo PhD), Department of Medicine (Prof K R Polkinghorne PhD, Prof A G Thrift PhD), School of Pharmacy (S W H Lee PhD), School of Public Health and Preventive Medicine (I N Ackerman PhD,

Prof F M Cicuttini PhD, S Li PhD), The School of Clinical Sciences at Monash Health (S Zaman MPH), Monash University, Melbourne, VIC, Australia; Department of Agriculture and Food Systems (H Suleria PhD), Department of General Practice (J Zhang MD), Department of Medicine (Prof B C Cowie PhD), Department of Neurology (Prof T Wijeratne MD), Department of Paediatrics (Prof G C Patton MD, Prof S M Sawyer MD), Melbourne Medical School (K L Chin PhD), Melbourne School of Population and Global Health (T Adair PhD, Prof A D Lopez PhD), School of Health Sciences (A Meretoja MD), University of Melbourne, Melbourne, VIC, Australia; Centre of Excellence for Epidemiological Modelling and Analysis (O O Adetokunboh PhD), Department of Global Health

(A A Adamu PhD, O O Adetokunboh PhD, C J Iwu PhD), Department of Psychiatry (Prof C D H Parry PhD, Prof S Seedat PhD), Stellenbosch University, Cape Town, South Africa; Alcohol, Tobacco \& Other Drug Research Unit (Prof C D H Parry PhD), Cochrane South Africa (A A Adamu PhD, D E Ndwandwe PhD), Grants, Innovation and Product Development Unit (P W Mahasha PhD), Risk and Resilience in Mental Disorders Research Unit (Prof D J Stein MD), South African Medical Research Council, Cape Town, South Africa (C J Iwu PhD, C A Nnaji MPH, E Z Sambala PhD, Prof C S Wiysonge MD); College of Medicine (O M Adebayo MD), Department of Community Medicine (O S Ilesanmi PhD), Department of Medicine (Prof M O Owolabi DrM), School of Laboratory Medicine and Science (A M Adeoye MD), University College Hospital, Ibadan, Ibadan, Nigeria; Department of Sociology (I A Adedeji PhD), Olabisi Onabanjo University, Ago-Iwoye, Nigeria; School of Medicine (V Adekanmbi PhD), Cardiff University, Cardiff, UK; Department of Community Medicine (O S Ilesanmi PhD), Department of Health Promotion and Education (S E Ibitoye MPH, Y O John-Akinola PhD, M M Oluwasanu PhD), Department of Medicine (Prof M O Owolabi DrM), Department of Obstetrics and Gynecology (O B Oghenetega MSc), Institute for Advanced Medical Research and Training (R O Akinyemi PhD), Institute of Cardiovascular Diseases (A M Adeoye MD), University of Ibadan, Ibadan, Nigeria; Department of Community Medicine (H Zandian PhD), Department of Environmental
Health Engineering (M Fazlzadeh PhD), School of Health (D Adham PhD), Social Determinants of Health Research Center (T Zahirian Moghadam PhD, H Zandian PhD), Ardabil University of Medical Science, Ardabil, Iran; Social Behavioral Research Branch (S M Advani PhD), National Eye Institute (W L Teagle BA), National Institute of Health, Bethesda, MD, USA; Department of Oncology (S M Advani PhD), Division of Hematology and Oncology (D Braithwaite PhD), Georgetown University, Washington DC, DC, USA; Department of Cardiovascular Medicine (P Agasthi MD, S Saadatagah MD), Department of Dermatology (M Afarideh MD), Mayo Clinic, Rochester, MN, USA; Department of Community Medicine (M Afshari MD), Zabol University of Medical Sciences, Zabol, Iran; Department of Global Public Health (E E Agardh PhD,

Prof P Allebeck MD, A Danielsson PhD, K Deuba DrPH), Department of Medical Epidemiology and Biostatistics (Prof J J Carrero PhD), Department of Medicine (D K Mohammad PhD), Department of Neurobiology (S Fereshtehnejad PhD), Department of Neurobiology, Care Sciences and Society (Prof J Ärnlöv PhD, C Nowak PhD), Department of Physiology and Pharmacology (C R Cederroth PhD), Department of Public Health (A Ahmadi MD), Department of Global Public Health-Health Systems and Policy (HSP): Medicines Focusing Antibiotics (Prof A Pathak PhD), Karolinska Institutet, Stockholm, Sweden; Department of Health Research Methods, Evidence and Impact (E J Mills PhD), Department of Medicine (O P Kurmi PhD), Department of Psychiatry and Behavioural Neurosciences (A T Olagunju MD), Department of Family Medicine (Prof G Agarwal PhD), Population Health Research Institute (T Sathish PhD), McMaster University, Hamilton, ON, Canada; Department of Epidemiology and Biostatistics (M Aghaali PhD, A Mohammadbeigi PhD), Neuroscience Research Center (A Mohammadbeigi PhD), Qom University of Medical Sciences, Qom, Iran; Institute of Genomics and Integrative Biology

(Prof A Agrawal PhD), Council of Scientific \& Industrial Research, Delhi, India; Internal Medicine (Prof A Agrawal PhD), Baylor College of Medicine, Houston, TX, USA; Department of Epidemiology and Health Statistics (T Ahmad MS), Southeast University, Nanjing, China; Lincoln Medical School (K Ahmadi PhD), Universities of Nottingham \& Lincoln, Lincoln, UK; Clinical Research Development Center (S Maleki MSc, M Naderi PhD), Department of Anatomical Sciences

(M R Salahshoor PhD), Department of Anesthesiology (A Ahmadi MD), Department of Emergency Medicine (R Pourmirza Kalhori PhD), Department of Environmental Health Engineering (Prof A Almasi PhD), Department of Epidemiology and Biostatistics (Y Salimi PhD), Department of Health Education and Health Promotion (S Siabani PhD, A Ziapour PhD), Department of Occupational Health Engineering (A Barzegar PhD), Department of Psychiatry (Prof H Khazaie MD), Department of Public Health (N Kianipour MA), Department of Radiology and Nuclear Medicine (F Amiri MSc, S Salehi Zahabi PhD), Department of Rehabilitation and Sports Medicine

(M Mirzaei MSc, M Shamsi PhD), Epidemiology and Biostatistics Department (Prof F Najafi PhD), Health Institute (A Jalali PhD), Infectious Disease Research Center (Prof K Ghadiri MD), Medical Biology Research Center (F Heydarpour PhD), Pediatric Department (Prof K Ghadiri MD), Research Center for Environmental Determinants of Health (N Fattahi PhD, Prof B Karami Matin PhD,

A Kazemi Karyani PhD, M Moradi PhD, Prof F Najafi PhD, Prof M Pirsaheb PhD, F Rajati PhD, Prof E Sadeghi PhD, Y Safari PhD, K Sharafi PhD, S Soltani PhD, Y Vasseghian PhD), Social Development and Health Promotion Research Center (Z Atafar PhD, F Jalilian PhD, M Mirzaei-Alavijeh PhD, S Saeidi MSc, Y Salimi PhD, M Soofi PhD, A Zangeneh MSc), Substance Abuse Prevention Research Center (A Jalali PhD, B Mansouri PhD), Kermanshah University of Medical Sciences, Kermanshah, Iran (H Janjani PhD); Environmental Health Engineering (M Ahmadi PhD), Environmental Technologies Research Center (M Ahmadi PhD), Social Determinants of Health Research Center (M A Khafaie PhD), Thalassemia and Hemoglobinopathy Research Center (F Rahim PhD), Ahvaz Jundishapur University of Medical Sciences, Ahvaz, Iran; Aging Research Institute (S Safiri PhD), Biotechnology Research Center (M Moghadaszadeh PhD), Department of Biostatistics and Epidemiology (Prof M Asghari Jafarabadi PhD), Department of Community Medicine (S Safiri PhD), Department of Health Policy and Management (L Doshmangir PhD), Department of 
Immunology (F Jadidi-Niaragh PhD), Department of Medical Surgical Nursing (M Ghafourifard PhD), Department of Parasitology and Mycology (A Spotin PhD), Department of Public Health (M Khodayari PhD), Drug Applied Research Center (H Samadi Kafil PhD), Health Services Management Research Center (L R Kalankesh PhD), Infectious and Tropical Diseases Research Center (E Ahmadpour PhD), Molecular Medicine Research Center (M Moghadaszadeh PhD, Prof B Yousefi PhD), Research Center for Evidence Based Medicine (F Ansari PhD), School of Nursing and Midwifery (H Hassankhani PhD), Social Determinants of Health Research Center (S Karimi PhD), Tabriz University of Medical Sciences, Tabriz, Iran (H Haririan PhD); Department of Environmental Health Sciences and Technology (S Mereta PhD), Department of Epidemiology (M B Ahmed MPH), Department of Health Education and Behavioral Sciences (G T Feyissa PhD), Jimma University, Jimma, Ethiopia; Australian Center for Precision Health (M B Ahmed MPH), School of Pharmacy and Medical Sciences (L G Ciobanu PhD), University of South Australia, Adelaide, SA, Australia; Faculty of Medicine and Public Health (B Aji DrPH), Jenderal Soedirman University, Purwokerto, Indonesia; Department of Clinical Midwifery (G L Aynalem MSc), Department of Clinical Pharmacy (B Gebresillassie MSc, Y G Tefera MSc), Department of Epidemiology and Biostatistics (T Y Akalu MPH, A G Baraki MPH, G A Tesema MPH, Z T Tessema MSc), Department of Midwifery (A T Tamiru MSc), Department of Reproductive Health (Z N Azene MPH), School of Nursing (H B Netsere MS), University of Gondar, Gondar, Ethiopia; Institute of Neuroscience (R O Akinyemi PhD), School of Medicine and Public Health (B Gebresillassie MSc), Newcastle University, Newcastle upon Tyne, UK; Department of Family Medicine and Community Health (Y Yano MD), Department of Population Health Sciences (T Akinyemiju PhD), Duke Global Health Institute (T Akinyemiju PhD, C Wu PhD, S Zadey MS), Department of Medicine (Prof Z Samad MD), Nicholas School of the Environment (J S Ji DSc), School of Medicine (P P Doshi MS), Duke University, Durham, NC, USA; Department of Medicine (O John MD), George Institute for Global Health (Prof V Jha MD), National Drug and Alcohol Research Centre (Prof L Degenhardt PhD), School of Medicine (P K Maulik PhD), School of Optometry and Vision Science (Prof K Pesudovs PhD, Prof S Resnikoff MD), School of Psychiatry (Prof P B Mitchell MD, Prof P S Sachdev MD), School of Public Health and Community Medicine (B Akombi PhD, M A Godinho MBBS, Prof R Q Ivers PhD, A E Peden PhD), Transport and Road Safety (TARS) Research Centre (R Biswas MSc, S Boufous PhD), University of New South Wales, Sydney, NSW, Australia; Department of Public Health (C J Akunna DMD), The Intercountry Centre for Oral Health (ICOH) for Africa, Jos, Nigeria; Department of Public Health (C J Akunna DMD), Federal Ministry of Health, Garki, Nigeria; Mayo Evidence-based Practice Center (F Alahdab MSc), Mayo Clinic Foundation for Medical Education and Research, Rochester, MN, USA; Department of Energy, Environmental and Chemical Engineering (M S Hammer PhD, A van Donkelaar PhD), John T. Milliken Department of Internal Medicine (Z Al-Aly MD), Washington University in St. Louis, St Louis, MO, USA; Clinical Epidemiology Center (Z Al-Aly MD), Dermatology Service (Prof R P Dellavalle MD), Department of Veterans Affairs, St Louis, MO, USA; Department of Community Health and Epidemiology (S Alam MSc), Department of Medicine (L E Cahill PhD), Department of Physics and Atmospheric Science (M S Hammer PhD, A van Donkelaar PhD, Prof R V Martin PhD), Dalhousie University, Halifax, NS, Canada; Prevention Division (N Alam MPH), Queensland Health, Brisbane, QLD, Australia; Australian Institute for Suicide Research and Prevention (Prof D De Leo DSc), Centre for Environment and Population Health (N Alam MPH), School of Dentistry and Oral Health (S K Tadakamadla PhD), Griffith University, Nathan, QLD, Australia; Murdoch Business School (K Alam PhD), Murdoch University, Perth, WA, Australia; Center for Health System Research (J E Alcalde-Rabanal PhD, L Avila-Burgos ScD, E Serván-Mori PhD), Center for Nutrition and Health Research (E Denova-Gutiérrez DSc, S Rodríguez-Ramírez DSc), Center for Poplulation Health Research (L Morales MSc), Department of Population Health Monitoring and Analysis (A Poznańska PhD, B Wojtyniak DSc), Director General (Prof J A Rivera PhD), Health and Nutrition Research Center
(I R Campos-Nonato PhD), National Institute of Public Health, Cuernavaca, Mexico (J Campuzano Rincon PhD, T G Sánchez-Pimienta MSc); Department of Midwifery (M B Mengesha MSc, F T Welay MSc), Department of Pharmacy (N M Alema MSc, D G Demsie MSc), Department of Public Health (H Teame MPH, B F Teklehaimanot MPH), Adigrat University, Adigrat, Ethiopia; College of Medicine and Health Science (B W Alemu MSc), Department of Midwifery (B W Alemu MSc, T Gultie MSc), Department of Nursing (A B Bante MSc, Y G Yeshitila MSc), Department of Public Health (Y C D Geramo MSc, M Glagn MPH, M B Sorrie MPH, F G W/hawariat MPH), Arba Minch University, Arba Minch, Ethiopia; College of Medicine and Health Science (A Amare PhD), Department of Adult Health Nursing (A Kasa MSc), Department of Epidemiology and Biostatistics (Y Alemu MPH, K M Mihretie MPH), Department of Health System and Health Economics (D D Atnafu MPH), Department of Medical Laboratory Sciences (F M Demeke MSc, A Melese MSc), Department of Midwifery (S A Bante MSc), Department of Nursing (G A Dessie MSc), Department of Reproductive Health and Population Studies (E W Mengesha MPH, D Nigatu MPH), School of Health Sciences, Department of Surgical Nursing (H B Netsere MS), Bahir Dar University, Bahir Dar, Ethiopia; College of Medicine

(M A H Almadi FRCPC), Department of Cardiac Sciences (Prof K F Alhabib MD), Internal Medicine Department (Y Mohammad MD), Pediatric Intensive Care Unit (K A Altirkawi MD, M Temsah MD), King Saud University, Riyadh, Saudi Arabia; Department of Family and Community Health (N Kugbey PhD), Department of Health Policy Planning and Management (M A Ayanore PhD), Institute of Health Research (R K Alhassan PhD), University of Health and Allied Sciences, Ho, Ghana; Department of Biotechnology (M Ali PhD), Quaid-i-Azam University, Islamabad, Pakistan; Department of Information Systems, College of Economics and Political Science (S Ali PhD), Sultan Qaboos University, Muscat, Oman; Directorate for Social Statistics and Population Census (G Alicandro PhD), Italian National Institute of Statistics, Rome, Italy; Health Services Management Department (R Kalhor PhD), Institute for Prevention of Non-communicable Diseases (R Kalhor PhD), Social Determinants of Health Research Center (M Alijanzadeh PhD), Qazvin University of Medical Sciences, Qazvin, Iran; Department of Health Care Management and Economics (C Alinia PhD, H Yusefzadeh PhD), Department of Public Health (A Didarloo PhD), Urmia University of Medical Science, Urmia, Iran; Infectious and Tropical Disease Research Center (H Alizade PhD), Hormozgan University of Medical Sciences, Bandar Abbas, Iran (A Nikpoor PhD); Department of Health Policy and Management (Prof S M Aljunid PhD), Kuwait University, Safat, Kuwait; International Centre for Casemix and Clinical Coding (Prof S M Aljunid PhD), National University of Malaysia, Bandar Tun Razak, Malaysia; Bordeaux School of Public Health (Prof F Alla PhD), University of Bordeaux, Bordeaux, France; Division of Gastroenterology \& Hepatology (M A H Almadi FRCPC), Epidemiology, Biostatistics and Occupational Health (L F Leal PhD), McGill University, Montreal, QC, Canada; Department of Epidemiology (A Almasi-Hashiani PhD, R Moradzadeh PhD, M Zamanian PhD), Department of Pediatrics (J Nazari MD), Health Services Management Department (S Amini PhD), Occupational Health Department (F Ghamari PhD), Arak University of Medical Sciences, Arak, Iran; Physiotherapy Department (Prof N A Almasri PhD), The University of Jordan, Amman, Jordan; Epidemiology Department (M Khan MD), Medical Research Center (H M Al-Mekhlafi PhD), Jazan University, Jazan, Saudi Arabia (Prof N Bedi MD); Department of Parasitology (H M Al-Mekhlafi PhD), Sana'a University, Sana'a, Yemen; Research Program of Epidemiology and Public Health (J Alonso MD), Pompeu Fabra University, Barcelona, Spain; Department of Experimental and Health Sciences (J Alonso MD), Biomedical Research Networking Center in Epidemiology and Public Health (CiberESP), Madrid, Spain; Department of Community Medicine (R M Al-Raddadi PhD), King Abdulaziz University, Jeddah, Saudi Arabia; Institute for Immunological Research (Prof J Zakzuk PhD), Research Group in Health Economics (Prof N Alvis-Guzman PhD), University of Cartagena, Cartagena, Colombia; Research Group in Hospital Management and Health Policies (Prof N Alvis-Guzman PhD), Universidad de la Costa, Barranquilla, Colombia; Department of Economic Sciences (N J Alvis-Zakzuk MSc), University of the Coast, 
Barranquilla, Colombia; Colombian National Health Observatory (N J Alvis-Zakzuk MSc, C A Castañeda-Orjuela MD), Department of Public Health Research (J N Malagón-Rojas MSc), National Institute of Health, Bogota, Colombia; Department of Biostatistics (K Gezae MSc), Department of Epidemiology (H A Gesesew PhD), Department of Microbiology and Immunology (S Muthupandian PhD), Department of Nursing (G G Gebremeskel MSc), Department of Nutrition and Dietetics (K Berhe MPH), Department of Pharmacology (B Amare MSc), Department of Pharmacology and Toxicology (M A Ayza MSc), Department of Pharmacy (L G Gebremeskel MSc), Department of Reproductive Health (A A A Gebreslassie MPH), School of Pharmacy (H Bitew MSc, K A Zewdie MSc), School of Public Health (F G G Tela BHlthSci), Mekelle University, Mekelle, Ethiopia; Adelaide Medical School (L G Ciobanu PhD, T K Gill PhD), Centre for Heart Rhythm Disorders (J Noubiap MD), Robinson Research Institute (Z S Lassi PhD), School of Medicine (A Amare PhD), School of Public Health (D Bhandari MSc), University of Adelaide, Adelaide, SA, Australia; Department of Epidemiology and Biostatistics (A L Amit BS), Department of Health Policy and Administration (C T Antonio MD, E A Faraon MD), Department of Nutrition (J F Lopez MD), University of the Philippines Manila, Manila, Philippines; Center for Clinical Global Health Education (S R Atre PhD), Department of Cardiology (M Shabani MD), Department of Epidemiology (S H Ballew PhD, Prof K Matsushita MD), Department of Health Policy and Management (A T Khoja MD), Department of Pathology (P Daneshpajouhnejad MD), Department of Radiology and Radiological Science (M Ghadimi MD, N Hafezi-Nejad MD, A Haj-Mirzaian MD, O Shafaat MD), School of Advanced International Studies (Prof J Fanzo PhD), School of Public Health (A L Amit BS), Johns Hopkins University, Baltimore, MD, USA; Maternal and Child Wellbeing (D A Amugsi PhD), African Population and Health Research Center, Nairobi, Kenya; Lee Kuan Yew School of Public Policy (G H Amul MSc), Saw Swee Hock School of Public Health (Prof D S Q Koh PhD), Yong Loo Lin School of Medicine (Prof N Venketasubramanian MBBS), National University of Singapore, Singapore, Singapore; Department of Public Health (E W Anbesu MPH, J A Mohammed MPH, T G Wondmeneh MPH), Samara University, Samara, Ethiopia; Cardiology Department (C Andrei PhD), Department of Anatomy and Embryology (R I Negoi PhD), Department of General Surgery (D V Davitoiu PhD, A Manda MD, I Negoi PhD), Department of Infectious Diseases (L Preotescu PhD), Department of Internal Medicine (M Hostiuc PhD), Department of Legal Medicine and Bioethics (S Hostiuc PhD), Pharmacy Department (Prof R Ancuceanu PhD), Carol Davila University of Medicine and Pharmacy, Bucharest, Romania; ARC Centre of Excellence for Children and Families over the Life Course (M Hasan MPH), Center for Youth Substance Abuse Research (J Leung PhD), Centre for Sensorimotor Performance (D Anderlini MD), Institute for Social Science Research (M Hasan MPH, A A Mamun PhD, J C Maravilla PhD), Queensland Brain Institute (Prof J J McGrath MD), Queensland Centre for Mental Health Research (F J Charlson PhD), School of Dentistry (R Lalloo PhD), School of Health and Rehabilitation Sciences (R Uddin PhD), School of Public Health (H E Erskine PhD, A J Ferrari PhD, L D Knibbs PhD, A M Mantilla Herrera PhD, D F Santomauro PhD, J Shadid BSc, Prof H A Whiteford PhD), The University of Queensland, Brisbane, QLD, Australia; Neurology Department (D Anderlini MD), Royal Brisbane and Women's Hospital, Brisbane, QLD, Australia; Department of Statistics and Econometrics (A Mirica PhD, Prof M Ausloos PhD, Prof C Herteliu PhD, A Oțoiu PhD, A Pana MD, Prof T Andrei PhD), Management Department (S Ștefan PhD), Bucharest University of Economic Studies, Bucharest, Romania; Department of Medicine (S Androudi PhD), University of Thessaly, Volos, Greece; School of Health and Related Research (C Angus MSc), University of Sheffield, Sheffield, UK; Razi Vaccine and Serum Research Institute (F Ansari PhD), Agricultural Research, Education, and Extension Organization (AREEO), Tehran, Iran; Medical Students Research Committee (I Ansari MD), Shahed University, Tehran, Iran; Dietary Supplements and Probiotic Research Center (H Pourjafar PhD), School of Medicine (M Shams-Beyranvand MSc), Alborz University of Medical Sciences, Karaj, Iran; Department of Epidemiology and Biostatistics (Prof A Ansari-Moghaddam PhD), Health Promotion Research Center (M Khammarnia PhD), Zahedan University of Medical Sciences,
Zahedan, Iran; Research Center on Public Health (I Antonazzo PhD, P Ferrara MD), School of Medicine and Surgery (S Conti PhD, P A Cortesi PhD, C Fornari PhD), University of Milan Bicocca, Monza, Italy; Department of Applied Social Sciences (C T Antonio MD), School of Nursing (P H Lee PhD), Hong Kong Polytechnic University, Hong Kong, China; Agribusiness Study Program (E Antriyandarti DrAgrSc), Sebelas Maret University, Surakarta, Indonesia; Department of Environmental Health (Prof Z Yousefi PhD), Department of Immunology (Prof A Rafiei PhD), Department of Medical Mycology (J Javidnia PhD), Department of Medical Parasitology (Prof R Faridnia PhD), Department of Parasitology (D Anvari PhD), Faculty of Nursing and Midwifery (A Goudarzian MSc), Health Sciences Research Center (M Moosazadeh PhD), Molecular and Cell Biology Research Center (Prof A Rafiei PhD), Pediatric Infectious Diseases Research Center (Prof M Rezai MD), Toxoplasmosis Research Center (Prof A Daryani PhD), Mazandaran University of Medical Sciences, Sari, Iran; Department of Parasitology (D Anvari PhD), Iranshahr University of Medical Sciences, Iranshahr, Iran; College of Medicine (M Fareed PhD), Department of Pathology (R Anwer PhD), Department of Public Health (A T Khoja MD), Imam Mohammad Ibn Saud Islamic University, Riyadh, Saudi Arabia; Department of Sociology and Social Work (S Appiah PhD), Kwame Nkrumah University of Science and Technology, Kumasi, Ghana; Center for International Health (S Appiah PhD, D Paudel PhD), Institute for Stroke and Dementia Research (Prof M Dichgans MD), Ludwig Maximilians University, Munich, Germany; Cardiovascular Diseases Research Center (S Riahi PhD), Faculty of Medicine (A Rajabpour-Sanati MD), Social Determinants of Health Research Center (M Arab-Zozani PhD), Birjand University of Medical Sciences, Birjand, Iran; Department of Nursing (A A K Arba MSc), Wolaita Sodo University, Wolaita Sodo, Ethiopia; Department of Public Health (O Aremu PhD), Birmingham City University, Birmingham, UK; Regional Centre for the Analysis of Data on Occupational and Work-related Injuries and Diseases (F Ariani MSc), Local Health Unit Tuscany Centre, Florence, Italy; Public Health and Healthcare Management (T Aripov PhD), Tashkent Institute of Postgraduate Medical Education, Tashkent, Uzbekistan; Boston Children's Hospital, Boston, MA, USA (T Aripov PhD); Social Determinants of Health Research Center (B Armoon PhD,

M M Mahdavi PhD), Saveh University of Medical Sciences, Saveh, Iran; Social Determinants of Health Research Center (B Armoon PhD), Yasuj University of Medical Sciences, Yasuj, Iran; School of Health and Social Studies (Prof J Ärnlöv PhD), Dalarna University, Falun, Sweden; Department of Epidemiology and Public Health

(O O Arowosegbe MPH), Swiss Tropical and Public Health Institute, Basel, Switzerland; Department of Epidemiology and Public Health (O O Arowosegbe MPH), University of Basel, Basel, Switzerland (F Tediosi PhD); Monitoring Evaluation and Operational Research Project (K K Aryal PhD), Abt Associates Nepal, Lalitpur, Nepal; Department of Clinical Biochemistry (A Mosapour PhD), General Education Department (M Yaminfirooz PhD), School of Nursing and Midwifery (A Arzani DrPH), Social Determinants of Health Research Center (A Bijani PhD, M A Jahani PhD, S Mouodi PhD, M Yaminfirooz PhD), Student Research Committee (M Zamani MD), Babol University of Medical Sciences, Babol, Iran (A Arzani DrPH); Department of Plastic Surgery (M Asaad MD), University of Texas, Houston, TX, USA; Department of Epidemiology (M Dianatinasab MSc), Department of Healthcare Management and Education (M Bahrami PhD), Department of Parasitology and Mycology (Prof K Pakshir PhD), Epilepsy Research Center

(Prof A A Asadi-Pooya MD), Health Human Resources Research Center (M Bayati PhD), Health Policy Research Center (S Shahabi PhD), Non-communicable Disease Research Center (Prof R Malekzadeh MD, S G Sepanlou MD), Shiraz University of Medical Sciences, Shiraz, Iran; Neurology Department (Prof A A Asadi-Pooya MD), Thomas Jefferson University, Philadelphia, PA, USA; Department of Biostatistics and Epidemiology (Prof M Asghari Jafarabadi PhD), Department of Immunology (S Athari MPH), Zanjan University of Medical Sciences, Zanjan, Iran; Faculty of Nursing (M M W Atout PhD), Philadelphia University, Amman, Jordan; D.Y. Patil Medical College, Hospital and Research Centre (S R Atre PhD), D.Y. Patil Vidyapeeth, Pune, Pune, India; Department of Social Welfare (M S Atteraya PhD), Keimyung 
University, Daegu, South Korea; Department of Health Sciences (Prof T Brugha MD), School of Business (Prof M Ausloos PhD), University of Leicester, Leicester, UK; Gastro-enterology Department (F Ausloos MD), University of Liège, Liège, Belgium; Epidemiological and Clinical Research (E F G Avokpaho MD), Bénin Clinical Research Institute (IRCB), Abomey-Calavi, Benin; Epidemiology and Clinical Research (E F G Avokpaho MD), Foundation for Scientific Research (FORS), Cotonou, Benin; Centre for Alcohol Policy Research (CAPR) (Prof R Room PhD), Department of Public Health (M Islam PhD), School of Nursing and Midwifery (Prof D Edvardsson PhD, M Rahman PhD), The Judith Lumley Centre (B Ayala Quintanilla PhD), La Trobe University, Melbourne, VIC, Australia; Department of Public Health (Prof P W Gething PhD), School of Physiotherapy and Exercise Science (Prof A M Briggs PhD), School of Public Health (G Ayano MSc, D Hendrie PhD, T R Miller PhD), Curtin University, Perth, WA, Australia; Global Adolescent Health Group (P S Azzopardi PhD), Burnet Institute, Melbourne, VIC, Australia; Wardliparingga Aboriginal Research Unit (P S Azzopardi PhD), South Australian Health and Medical Research Institute, Adelaide, SA, Australia; Centre for Bio Cultural Studies (CBiCS) (P Hoogar PhD), Department of Community Medicine (D B B MD, V Kulkarni MD, N Kumar MD, P Mithra MD R S Shetty MD, R Thapar MD), Department of Forensic Medicine (J Padubidri MD), Department of Forensic Medicine and Toxicology (S M Bakkannavar MD, Prof V C Nayak MD, Prof P Rastogi MD, Prof B K Shetty MD), Department of Health Information Management (R Bhageerathy PhD), Department of Health Policy (N Gudi MPH), Department of Internal Medicine (A Boloor MD), Kasturba Medical College (R Holla MD, P Rathi MD, Prof B Unnikrishnan MD), Manipal College of Pharmaceutical Sciences (Prof M D Janodia PhD), Manipal Academy of Higher Education, Mangalore, India (R Bhageerathy PhD, Prof V Jha MD); Centre for Surveillance and Applied Research (J J Lang PhD), Public Health Risk Sciences Division (A Badawi PhD), Substance Related Harms Division (H M Orpana PhD), Public Health Agency of Canada, Toronto, ON, Canada; Centre for Global Child Health (Prof Z A Bhutta PhD), Dalla Lana School of Public Health (K D Shield PhD), Department of Medicine (V Chattu MD), Department of Nutritional Sciences (A Badawi PhD), University of Toronto, Toronto, ON, Canada; Department of Forensic Science (A D Badiye MSc, N Kapoor MSc), Government Institute of Forensic Science, Nagpur, India; Chemistry Department (Prof M Bagherzadeh PhD, N Rabiee MSc), Sharif University of Technology, Tehran, Iran; Department of Ophthalmology (E Bagli PhD), University Hospital of Ioannina, Ioannina, Greece; Institute of Molecular Biology \& Biotechnology (E Bagli PhD), Foundation for Research \& Technology, Ioannina, Greece; Unit of Biochemistry (A A Baig PhD), Sultan Zainal Abidin University, Kuala Terengganu, Malaysia; Centre for Community Medicine (M Bairwa MD), Department of Paediatrics (Prof R Lodha MD), Department of Psychiatry (Prof R Sagar MD), Department of Radiation Oncology (Prof G K Rath MD), All India Institute of Medical Sciences, New Delhi, India; Department of Community Medicine and Family Medicine (P Bhardwaj MD), Department of Pharmacology (J Charan MD), Department of Forensic Medicine and Toxicology (T Kanchan MD), All India Institute of Medical Sciences, Jodhpur, India; Department of Community Medicine and Family Medicine (A Joshi MD, A P Pakhare MD), All India Institute of Medical Sciences, Bhopal, India; Department of Radiation Oncology, All India Institute of Medical Sciences, New Delhi, India (Prof G K Rath MD); Department of Anesthesiology (M Bakhshaei MD), Department of Cardiology (B Naghshtabrizi MD), Department of Neuroscience (S Karimi PhD), Department of Physiology (S Karimi PhD), Hamedan University of Medical Sciences, Hamadan, Iran; Department of Demography (A Balachandran MSc), Department of Psychiatry (Prof H W Hoek MD), School of Economics and Business (Prof M J Postma PhD), University Medical Center Groningen (Prof M J Postma PhD), University of Groningen, Groningen, Netherlands; Population Research Centre (A Balachandran MSc), Institute for Social and Economic Change, Bengaluru, India; Department of Environmental Health (A Geremew PhD), Department of Medical Laboratory Sciences (A M Gebrehiwot PhD), Department of Medical Microbiology (S Balakrishnan PhD), School of Nursing and Midwifery (A Desalew MSc), School of Pharmacy (H A Bojia BPharm,
A S Mohammed BA), School of Public Health (L D Regassa MPH), Haramaya University, Harar, Ethiopia; Department of Biostatistics and Epidemiology (P G K Parmar PhD), Department of Psychology and Neurosciences (S Balalla PhD), National Institute for Stroke and Applied Neurosciences (Prof V L Feigin PhD, P G K Parmar PhD, S Roberts MSc), Auckland University of Technology, Auckland, New Zealand; Epidemiology Department (A Baldasseroni MD), Local Health Authority of Florence, Firenze, Italy; Department of Psychology (M A Stokes PhD), Institute for Physical Activity and Nutrition (Prof K Ball PhD, S Islam PhD, Prof R Maddison PhD, R Uddin PhD), Deakin University, Burwood, VIC, Australia; Department of Prevention (D Balzi DSc, M Levi PhD), USL Tuscany Center, Florence, Italy; Department of Hypertension (Prof M Banach PhD), Medical University of Lodz, Lodz, Poland; Polish Mothers' Memorial Hospital Research Institute, Lodz, Poland (Prof M Banach PhD); School of Health Sciences (Prof S K Banerjee PhD), Walden University, Minneapolis, MN, USA; Department of Non-communicable Diseases (P C Banik MPhil, L Barua MPH, M Faruque MPH), Bangladesh University of Health Sciences, Dhaka, Bangladesh; Department of Neurosciences (Prof M A Barboza MD), Costa Rican Department of Social Security, San Jose, Costa Rica; School of Medicine (Prof M A Barboza MD), University of Costa Rica, San Pedro, Costa Rica; School of Psychology (Prof S L Barker-Collo PhD), University of Auckland, Auckland, New Zealand; Department of Ophthalmology (Prof J B Jonas MD, S Panda-Jonas MD), Heidelberg Institute of Global Health (HIGH) (Prof T W Bärnighausen MD, J De Neve MD, S Kohler MD, B Moazen MSc, S Mohammed PhD), Heidelberg University, Heidelberg, Germany; Department of Industrial Engineering (Prof L H Barrero DSc), Pontifical Javeriana University, Bogota, Colombia; School of Public Health and Community Medicine (Prof H Basaleem PhD), Aden College, Aden, Yemen; Barcelona Institute for Global Health (Prof Q Bassat MD), Research Unit (J M Haro MD), University of Barcelona, Barcelona, Spain; Catalan Institution for Research and Advanced Studies (ICREA), Barcelona, Spain (Prof Q Bassat MD, A Koyanagi MD); Department of Primary Care and Public Health (J Car PhD, F Greaves PhD, Prof A Majeed MD), Department of Surgery and Cancer (Prof A C Davis PhD), Division of Brain Sciences (Prof T J Steiner PhD), Imperial College Business School (D Kusuma DSc), Department of Primary Care and Public Health (R Palladino MD, Prof S Rawaf MD), School of Public Health (S Basu PhD, Prof S Saxena MD), WHO Collaborating Centre for Public Health Education and Training (D L Rawaf MD), Imperial College London, London, UK; Department of Psychiatry (Prof B T Baune PhD), Institute for Epidemiology and Social Medicine (A Karch MD), University of Münster, Münster, Germany; Department of Psychiatry (Prof B T Baune PhD), Melbourne Medical School, Melbourne, VIC, Australia; Department of Public Health (B A Baye MPH, J Darega Gela MPH, T Kolola MPH), Ambo University, Ambo, Ethiopia; Department of Environmental Sciences and Engineering (J S Becker BA M DeLang BS, Prof J West PhD), Gillings School of Global Public Health (M M Gad MD), Department of Environmental Sciences and Engineering (M L Serre PhD), University of North Carolina Chapel Hill, Chapel Hill, NC, USA; Department of Community Medicine (Prof N Bedi MD), Gandhi Medical College Bhopal, Bhopal, India; Department of Environmental Health Sciences (S Gallus DSc, A Lugo PhD), Department of Neuroscience (E Beghi MD, E Pupillo PharmD), Department of Oncology (C Bosetti PhD), Laboratory of Neurological Disorders (G Giussani PhD), Mario Negri Institute for Pharmacological Research, Milan, Italy (B Bikbov MD, N Perico MD, Prof G Remuzzi MD); Department of Public Health (M Imani-Nasab PhD, S Nouraei Motlagh PhD), Razi Herbal Medicines Research Center (Z Sharafi PhD), Social Determinants of Health Research Center (M Behzadifar PhD), Lorestan University of Medical Sciences, Khorramabad, Iran; Department of Neurology (Prof Y Béjot PhD), University Hospital of Dijon, Dijon, France; Dijon Stroke Registry - UFR Sciences Santé (Prof Y Béjot PhD), University of Burgundy, Dijon, France; Department of Public Health (T T Bekuma MPH, M T Yilma MPH), Wollega University, Nekemte, Ethiopia; School of the Environment (Prof M L Bell PhD), Yale University, New Haven, CT, USA; Department of Medicine (A K Bello PhD, A Rafiee MSc), University of Alberta, Edmonton, AB, 
Canada; Big Data Institute (S H Keddie MSc, T C D Lucas PhD), Centre for Tropical Medicine and Global Health (C Dolecek MD, S J Dunachie PhD), Department of Zoology (M U G Kraemer PhD), Malaria Atlas Project (M Nguyen PhD, S F Rumisha PhD,

D J Weiss PhD), Medical Research Council Population Health Research Unit (Prof S Lewington PhD), Nuffield Department of Medicine (E L Collins MSc, A K Nandi PhD, Y Zhao MPH), Nuffield Department of Population Health (D A Bennett PhD, M A Karim MD, B Lacey PhD, Prof S Lewington PhD), Nuffield Department of Surgical Sciences (R Khundkar MA), The George Institute for Global Health (Prof S Yaya PhD), University of Oxford, Oxford, UK; Center for Clinical and Epidemiological Research (A C Goulart PhD, I S Santos PhD), Department of Internal Medicine (I M Bensenor PhD, A C Goulart PhD, I S Santos PhD), Department of Medicine (Prof P A Lotufo DrPH), Department of Pathology and Legal Medicine (M R Tovani-Palone PhD), Department of Psychiatry (Prof J Castaldelli-Maia PhD, Y Wang PhD), Division of Ophthalmology (J M Furtado MD), Laboratory of Genetics and Molecular Cardiology (A Pereira PhD), University of São Paulo, São Paulo, Brazil; Heart and Vascular Center (C P Benziger MD), Essentia Health, Duluth, MN, USA; Department of Medicine (A E Berman MD), Medical College of Georgia at Augusta University, Augusta, GA, USA; Department of Twin Research and Genetic Epidemiology (M Mazidi PhD), Faculty of Dentistry, Oral \& Craniofacial Sciences (E Bernabe PhD), Faculty of Life Sciences and Medicine (Prof P I Dargan MB, M Molokhia PhD), Institute for Population Health (Prof K Shibuya MD), School of Population Health and Environmental Sciences (A Douiri PhD, Y Wang PhD, Prof C D A Wolfe MD), St John's Institute of Dermatology (Prof R J Hay MD), Unit for Population-Based Dermatology Research (Prof C Flohr PhD), King's College London, London, UK; Department of Pediatrics (Prof P S Suchdev MD), Division of Cardiology (P Ram MD), Hubert Department of Global Health (R S Bernstein MD), Rollins School of Public Health (Prof Y Liu PhD), Emory University, Atlanta, GA, USA; Department of Internal Medicine (A S Bhagavathula PharmD), Department of Medical Microbiology \& Immunology (Prof G Khan PhD), Institute of Public Health, College of Medicine and Health Sciences (Prof M Grivna PhD),

United Arab Emirates University, Al Ain, United Arab Emirates; Department of Public Health and Preventive Medicine (Prof M Grivna PhD), Department of Social and Clinical Pharmacy (A S Bhagavathula PharmD), Charles University, Prague, Czech Republic; Institutes of Applied Health Research and Translational Medicine (N Bhala PhD), Queen Elizabeth Hospital Birmingham, Birmingham, UK; Institute of Applied Health Research (N Bhala PhD, J S Chandan MFPH), Institute of Occupational and Environmental Medicine (O P Kurmi PhD), University of Birmingham, Birmingham, UK; Public Health Research Laboratory (D Bhandari MSc), Tribhuvan University, Kathmandu, Nepal; Department of Community Medicine (Prof A M Gaidhane MD, Prof Z Quazi Syed PhD, Prof D Saxena PhD), Global Evidence Synthesis Initiative (Prof M Khatib PhD), Datta Meghe Institute of Medical Sciences, Wardha, India; Division of General Internal Medicine (A G Bhat MD), University of Massachusetts Medical School, Springfield, MA, USA; Department of Statistical and Computational Genomics (K Bhattacharyya MSc), National Institute of Biomedical Genomics, Kalyani, India; Department of Statistics (K Bhattacharyya MSc), University of Calcutta, Kolkata, India; Department of Global Health (S Bhattarai MD), Global Institute for Interdisciplinary Studies, Kathmandu, Nepal; Centre of Excellence in Women \& Child Health (Prof Z A Bhutta PhD), Department of Medicine (Prof Z Samad MD), Division of Women and Child Health (J K Das MD), Aga Khan University, Karachi, Pakistan; Institute of Soil and Environmental Sciences (S Bibi PhD), University of Agriculture Faisalabad, Faisalabad, Pakistan; Health Economics and Outcomes Research (V Bilano PhD), Creativ-Ceutical, London, UK; National Centre for Epidemiology and Population Health (M Bin Sayeed MSc), Research School of Population Health (Prof N Cherbuin PhD), Australian National University, Canberra, ACT, Australia; Department of Clinical Pharmacy and Pharmacology (M Bin Sayeed MSc), University of Dhaka, Dhaka, Bangladesh; Department “G.F.Ingrassia” (E D’Amico MD), Department of Biomedical and Biotechnological Sciences (G Grosso PhD), Department of Clinical and Experimental Medicine (A M Borzì MD), Department of General Surgery and Medical-Surgical Specialties
(Prof A Biondi PhD, M Vacante PhD, A Valli PhD), University of Catania, Catania, Italy; Ethiopian Public Health Institute, Adis Ababa, Ethiopia (B M Birihane MSc); Department of Nursing (B M Birihane MSc), Debre Tabor University, Debretabor, Ethiopia; Global Health Division (D Bisanzio PhD), Research Triangle Institute International, Research Triangle Park, NC, USA; National Institute of Health Research Nottingham Biomedical Research Centre (C R Cederroth PhD), School of Medicine (D Bisanzio PhD), University of Nottingham, Nottingham, UK; Center for International Health (CIH) and Bergen Center for Ethics and Priority Setting (BCEPS) (S Hassan MPhil), Department of Global Public Health and Primary Care (Prof T Bjørge PhD, Prof O F Norheim PhD), Department of Psychosocial Science (Prof S Øverland PhD), University of Bergen, Bergen, Norway; Cancer Registry of Norway, Oslo, Norway (Prof T Bjørge PhD); European \& Developing Countries Clinical Trials Partnership, Cape Town, South Africa (Prof M J Bockarie MSc); Department of Medicine (Prof M J Bockarie MSc, G A Mensah MD), Hatter Institute Department of Medicine (Prof K Sliwa PhD), School of Public Health and Family Medicine (C A Nnaji MPH,

Prof C S Wiysonge MD), University of Cape Town, Cape Town, South Africa; Department of Biology (A Gholamian MSc), Department of Veterinary Medicine (S Bohlouli PhD), Tehran Medical Sciences Branch (S Esmaeilnejad PhD), Young Researchers and Elite Club (A Gholamian MSc), Islamic Azad University, Kermanshah, Iran; Department of Computer Science and Information Technology (M Bohluli PhD), Institute for Advanced Studies in Basic Sciences, Zanjan, Iran; Department of Research and Innovation (M Bohluli PhD), Petanux Research GmBH, Bonn, Germany; Department of Biomedical Sciences (S R Bolla PhD), Nazarbayev University, Nur-Sultan City, Kazakhstan; Department of Epidemiology and Psychosocial Research (Prof G Borges DSc), Ramón de la Fuente Muñiz National Institute of Psychiatry, Mexico City, Mexico; Transport Global Practice (D Bose PhD), World Bank, Washington, DC, USA; Vision and Eye Research Unit (Prof R Bourne MD), Anglia Ruskin University, Cambridge, UK; Department of Public Health and Primary Care (Prof C Brayne MD), University of Cambridge, Cambridge, UK; Department of Psychology (Prof N J K Breitborde PhD), James Cancer Hospital (J L Fisher PhD), Psychiatry and Behavioral Health Department

(Prof N J K Breitborde PhD), Ohio State University, Columbus, $\mathrm{OH}$, USA; Institute for Medical Information Processing, Biometry, and Epidemiology (S Breitner DSc), Ludwig Maximilian University of Munich, Munich, Germany; Institute of Epidemiology (S Breitner DSc), German Research Center for Environmental Health, Neuherberg, Germany; Division of Clinical Epidemiology and Aging Research (Prof H Brenner MD), German Cancer Research Center, Heidelberg, Germany; Institute of Medicine (Prof A V Breusov DSc), RUDN University, Moscow, Russia; European Office for the Prevention and Control of Noncommunicable Diseases (I Rakovac PhD), World Health Organization (WHO), Geneva, Switzerland; Department of Epidemiology and Evidence Based Medicine (Prof N I Briko DSc, P D Lopukhov PhD), Department of Epidemiology and Evidence-Based Medicine (E V Glushkova PhD, V A Korshunov PhD, R V Polibin PhD), N. A. Semashko Department of Public Health and Healthcare (Prof M Jakovljevic PhD), I.M. Sechenov First Moscow State Medical University, Moscow, Russia; Department of Biomedical Technologies (A N Briko MSc), Bauman Moscow State Technical University, Moscow, Russia; Neuroscience Unit (G B Britton PhD), Institute for Scientific Research and High Technology Services, Panama City, Panama; Gorgas Memorial Institute for Health Studies, Panama, Panama (G B Britton PhD, F Castro MD, I Moreno Velásquez PhD); Monash Department of Clinical Epidemiology at Cabrini Hospital (Prof R Buchbinder PhD), Cabrini Institute, Melbourne, VIC, Australia; Population Studies Division (R T Burnett PhD), Health Canada, Ottawa, ON, Canada; Department of Community Medicine

(Prof S Burugina Nagaraja MD), Employee State Insurance Post Graduate Institute of Medical Sciences and Research, Bengalore, India; Department of Health Care Management (Prof R Busse PhD), Technical University of Berlin, Berlin, Germany; School of Public Health and Health Systems (Z A Butt PhD), University of Waterloo, Waterloo, ON, Canada; Al Shifa School of Public Health (Z A Butt PhD), Al Shifa Trust Eye Hospital, Rawalpindi, Pakistan; Institute of Microengineering 
(F Caetano dos Santos PhD), Federal Polytechnic School of Lausanne, Lausanne, Switzerland; Department of Sociology (Prof T Cai PhD), Department of Psychology (B J Hall PhD), University of Macau, Macau, China; School of Public Health and Administration

(L Cahuana-Hurtado PhD), Peruvian University Cayetano Heredia, Lima, Peru; Internal Medicine Department (Prof L A Cámera MD), Hospital Italiano de Buenos Aires, Buenos Aires, Argentina; Board of Directors (Prof L A Cámera MD), Argentine Society of Medicine, Buenos Aires, Argentina (Prof P R Valdez MEd); School of Medicine

(J Campuzano Rincon PhD), University of the Valley of Cuernavaca, Cuernavaca, Mexico; Centre for Population Health Sciences (J Car PhD), Lee Kong Chian School of Medicine (L Tudor Car PhD), Nanyang Technological University, Singapore, Singapore; Department of Health Care (Prof R Cárdenas DSc), Metropolitan Autonomous University, Mexico City, Mexico; Oncological Network, Prevention and Research Institute (G Gorini MD), Institute for Cancer Research, Prevention and Clinical Network, Florence, Italy (G Carreras PhD); Associated Laboratory for Green Chemistry (LAQV) (Prof E Fernandes PhD, M Freitas PhD, Prof D M Pereira PhD), Center for Health Technology and Services Research (J V Santos MD), Department of Chemical Sciences (D Ribeiro PhD), Department of Chemistry (M Pinheiro PhD), Department of Clinical Neurosciences and Mental Health (J Massano MD), Institute for Research and Innovation in Health (J das Neves PhD), Institute of Biomedical Engineering (INEB) (J das Neves PhD), Laboratory of Toxicology (Prof D Dias da Silva PhD), Research Unit on Applied Molecular Biosciences (UCIBIO) (Prof F Carvalho PhD, V M Costa PhD, J P Silva PhD), University Hospital Center of Porto (J Morgado-da-Costa MSc), University of Porto, Porto, Portugal; Epidemiology and Public Health Evaluation Group (C A Castañeda-Orjuela MD), National University of Colombia, Bogota, Colombia; Department of Medicine (G Castelpietra PhD), University of Udine, Udine, Italy; Department of Mental Health (G Castelpietra PhD), Healthcare Agency "Friuli Occidentale", Pordenone, Italy; National School of Public Health (F Catalá-López PhD), Institute of Health Carlos III, Madrid, Spain; Clinical Epidemiology Program

(F Catalá-López PhD), Department of Medicine (S Pakhale MD), Ottawa Hospital Research Institute, Ottawa, ON, Canada; Mary MacKillop Institute for Health Research (Prof E Cerin PhD), Australian Catholic University, Melbourne, VIC, Australia; Centre for Suicide Research and Prevention (Prof P Yip PhD), Department of Social Work and Social Administration (Prof P Yip PhD), Department of Urban Planning and Design (H Ho PhD), School of Public Health (Prof E Cerin PhD), University of Hong Kong, Hong Kong, China; College of Medicine (J Chang PhD), National Taiwan University, Taipei, Taiwan; Department of Nursing (J Chang PhD), National Taiwan University Hospital, Taipei, Taiwan; Kidney Health Research Institute (A R Chang MD), Geisinger Health System, Danville, PA, USA; Cooperative Institute for Research in Environmental Sciences (K Chang PhD, O R Cooper PhD), University of Colorado, Boulder, CO, USA; Chemical Sciences Division (K Chang PhD), National Oceanic and Atmospheric Administration Earth System Research Laboratory, Boulder, CO, USA; Research Department (S Chaturvedi PhD), D.Y. Patil University, Pune, India; Institute of Industrial Ecological Science (O Chimed-Ochir PhD), University of Occupational and Environmental Health, Kitakyushu, Japan; Department of Public Health, Administration, and Social Sciences (J L Chirinos-Caceres DrPH), Cayetano Heredia University, Lima, Peru; Biomedical Informatics (J J Choi PhD), Seoul National University Hospital, Seoul, South Korea; Bispebjerg Hospital (Prof H Christensen DMSci), Rigshospitalet (T C Truelsen PhD), University of Copenhagen, Copenhagen, Denmark; Faculty of Biology (D Chu PhD), Hanoi National University of Education, Hanoi, Vietnam; Department of Epidemiology and Public Health (Prof M Kivimäki PhD), Department of Health Informatics (S Chung PhD), Division of Psychology and Language Sciences (M Kumar PhD), Ear Institute (Prof A C Davis PhD), Great Ormond Street Institute of Child Health (J L Ward MSc), University College London, London, UK; Health Data Research UK, London, UK (S Chung PhD); Department of Otolaryngology (M T Chung MD), Wayne State University, Detroit, MI, USA; Department of Public Health (Prof M Cirillo MD, Prof I Iavicoli PhD, R Palladino MD), University of Naples Federico II, Naples, Italy; Department of Key Questions and International
Cooperation (T K D Classen MSc), North Rhine-Westphalia (NRW) Centre for Health, Bochum, Germany; Bielefeld School of Public Health (T K D Classen MSc), Bielefeld University, Bielefeld, Germany; Health Effects Institute, Boston, MA, USA (A J Cohen DSc); National Oceanic and Atmospheric Administration Chemical Sciences Laboratory, Boulder, CO, USA (O R Cooper PhD); Institute of Clinical Physiology (P Lauriola MD), Institute of Neuroscience (B Corso PhD), National Research Council, Padova, Italy; Department of Preventive and Social Dentistry (F N Hugo PhD), Department of Psychiatry (C Kieling MD), Postgraduate Program in Epidemiology (E Cousin PhD,

Prof B B Duncan PhD, Prof B N G Goulart DSc, Prof M I Schmidt PhD, C Stein PhD), Federal University of Rio Grande do Sul, Porto Alegre, Brazil; Department of Psychology (R G Cowden PhD), University of the Free State, Park West, South Africa; Epidemiology

(J H MacLachlan MSc), WHO Collaborating Centre for Viral Hepatitis (Prof B C Cowie PhD), The Peter Doherty Institute for Infection and Immunity, Melbourne, VIC, Australia; Asbestos Diseases Research Institute (J Leigh MD), Faculty of Medicine and Health (M Cross PhD), Institute of Bone and Joint Research (Prof M L Ferreira PhD, E U R Smith PhD), Pain Management Research Institute (PMRI) (E U R Smith PhD), Sydney Medical School (S Islam PhD), Sydney School of Public Health (Prof T R Driscoll PhD, T M Huda PhD), University of Sydney, Sydney, NSW, Australia (D G Hoy PhD); Center for Translation Research and Implementation Science (G A Mensah MD), National Human Genome Research Institute (NHGRI) (N Horita PhD), Office of the Director (D H Cross PhD), National Institutes of Health, Bethesda, MD, USA; Department of Cardiology (H Dai MD), Department of Epidemiology and Health Statistics (Prof G Hu PhD), Central South University, Changsha, China; Department of Mathematics and Statistics (H Dai MD), York University, Toronto, ON, Canada; College of Environmental Sciences and Engineering (Prof H Dai PhD), Peking University, Beijing, China; Faculty of Medicine (Prof A A M Damasceno PhD), Eduardo Mondlane University, Maputo, Mozambique; Clinical Dermatology, IRCCS Istituto Ortopedico Galeazzi (G Damiani MD), Department of Clinical Sciences and Community Health (Prof C La Vecchia MD), University of Milan, Milan, Italy; Department of Dermatology (G Damiani MD), Department of Nutrition and Preventive Medicine (Prof J Sanabria MD), Case Western Reserve University, Cleveland, OH, USA; Department of Research (A Pandey PhD), Health Policy Research (M R Mathur PhD), Indian Institute of Public Health (Prof S Zodpey PhD), Public Health Foundation of India, Gurugram, India (Prof L Dandona MD, Prof R Dandona PhD, G Kumar PhD, D K Lal MD); Department of Clinical Toxicology (Prof P I Dargan MB), St John's Institute of Dermatology (Prof S M Langan PhD), Guy's and St. Thomas' NHS Foundation Trust, London, UK; College of Law and Political Science (K Hamagharib Abdullah PhD), Department of Computer Science (M Hosseinzadeh PhD), Department of Information Technology (A M Darwesh PhD), Department of Political Science (Prof A Faraj PhD), Diplomacy and Public Relations Department (A Omar Bali PhD), University of Human Development, Sulaymaniyah, Iraq; Department of Epidemiology and Biostatistics (R Das Gupta MPH), University of South Carolina, Columbia, SC, USA; James P Grant School of Public Health (R Das Gupta MPH), BRAC University, Dhaka, Bangladesh; Central University Tami Nadu, Thiruvarur, India (Prof A P Dash DSc); Department of Global Health and Infection (Prof G Davey MD), Wellcome Trust Brighton and Sussex Centre for Global Health Research (K Deribe PhD), Brighton and Sussex Medical School, Brighton, UK; Department of Nursing and Midwifery (K B Gebremedhin MSc), School of Nursing and Midwifery (A B Wondmieneh MSc), School of Public Health (Prof G Davey MD, N D Dereje PhD, K Deribe PhD), Addis Ababa University, Addis Ababa, Ethiopia; Department of Population and Development (C A Dávila-Cervantes PhD), Latin American Faculty of Social Sciences Mexico, Mexico City, Mexico; Department of Surgery (D V Davitoiu PhD), Clinical Emergency Hospital Sf. Pantelimon, Bucharest, Romania; Health Research Institute (K Davletov PhD, A Mereke MD), Al Farabi Kazakh National University, Almaty, Kazakhstan; Department of Dermatology

(Prof R P Dellavalle MD), School of Public Health (B Serdar PhD), University of Colorado Denver, Aurora, CO, USA; Department of Nursing (G G Gebremeskel MSc, T G Haile MSc, D B Tadesse MSc, 
G T Weldesamuel MSc), School of Pharmacy (G T Demoz MSc, L G Gebremeskel MSc, G Woldu MSc), Aksum University, Aksum, Ethiopia; Department of Public Health (N D Dereje PhD, D Handiso MPH), Wachemo University, Hossana, Ethiopia; St Paul's Eye Unit (N Dervenis MD), Royal Liverpool University Hospital, Liverpool, UK; 1st Department of Ophthalmology (Prof F Topouzis PhD), Department of Ophthalmology (N Dervenis MD), Aristotle University of Thessaloniki, Thessaloniki, Greece; Division of Cardiology (R Desai MBBS), Atlanta Veterans Affairs Medical Center, Decatur, GA, USA; Health, Nutrition, and HIV/AIDS Program (D Paudel PhD), National Centre for AIDS and STD Control (K Deuba DrPH), Save the Children, Kathmandu, Nepal; Department of Community Medicine (Prof S D Dharmaratne MD), University of Peradeniya, Peradeniya, Sri Lanka; Department of Microbiology (G P Dhungana MSc), Far Western University, Mahendranagar, Nepal; Department of Epidemiology and Biostatistics (M Dianatinasab MSc), Ophthalmic Epidemiology Research Center (M Emamian PhD), Shahroud University of Medical Sciences, Shahroud, Iran; Center of Complexity Sciences (Prof D Diaz PhD), National Autonomous University of Mexico, Mexico City, Mexico; Faculty of Veterinary Medicine and Zootechnics (Prof D Diaz PhD), Autonomous University of Sinaloa, Culiacan Rosales, Mexico; Swedish Family Medicine - First Hill, Seattle, WA, USA (M A Dirac MD); Development of Research and Technology Center (S Djalalinia PhD), Ministry of Health and Medical Education, Tehran, Iran; Center of Excellence in Behavioral Medicine (T H Nguyen BMedSc, G T Vu BA), Center of Excellence in Public Health Nutrition (H T Do MD), Nguyen Tat Thanh University, Ho Chi Minh City, Vietnam; Department of Social Medicine and Health Care Organisation (K Dokova PhD), Medical University of Varna, Varna, Bulgaria; Department of Population and Health (D T Doku PhD), University of Cape Coast, Cape Coast, Ghana; Department of Health Sciences (S Neupane PhD, Prof J S Takala DSc), University of Tampere, Tampere, Finland; Mahidol-Oxford Tropical Medicine Research Unit, Bangkok, Thailand (C Dolecek MD, S J Dunachie PhD); Urban Indian Health Institute (M C Doxey MPH), Seattle Indian Health Board, Seattle, WA, USA; School of Medicine (Prof K E Doyle PhD), School of Social Sciences and Psychology (Prof A M N Renzaho PhD), Translational Health Research Institute (F A Ogbo PhD, Prof A M N Renzaho PhD), Western Sydney University, Sydney, VIC, Australia; Department of Psychology (Prof S R Robinson PhD), Health Sciences (Prof K E Doyle PhD), Royal Melbourne Institute of Technology University, Melbourne, VIC, Australia; Department of Pediatric and Preventive Dentistry (A Vukovic DrPH), Faculty of Medicine (I M Ilic PhD, Prof M M Santric-Milicevic PhD), Faculty of Medicine Institute of Epidemiology (I S Vujcic PhD), Institute of Microbiology and Immunology (E Dubljanin PhD), School of Medicine (R Vukovic PhD), School of Public Health and Health Management (Prof M M Santric-Milicevic PhD), University of Belgrade, Belgrade, Serbia; Institute of Collective Health (Prof D Rasella PhD), School of Medicine (Prof A R Duraes PhD), Federal University of Bahia, Salvador, Brazil; Department of Internal Medicine (Prof A R Duraes PhD), Escola Bahiana de Medicina e Saúde Pública, Salvador, Brazil; Department of Social Services (A W Eagan MSW), Tufts Medical Center, Boston, MA, USA; Department of Epidemiology (M Ebrahimi Kalan MSc), Florida International University, Miami, FL, USA; Department of Nursing (Prof D Edvardsson PhD), Umeå University, Umea, Sweden; Centre Clinical Epidemiology and Biostatistics (A Effiong MB), Discipline of General Practice (Prof C D Pond PhD), University of Newcastle, Newcastle, NSW, Australia; Department of Ophthalmology and Visual Sciences (J R Ehrlich MD), Department of Systems, Populations, and Leadership (X Liu PhD), Institute for Health Care Policy and Innovation (J R Ehrlich MD), Medical School (M Y W Wei MD), University of Michigan, Ann Arbor, MI, USA; Biomedical Informatics and Medical Statistics Department (I El Sayed PhD), Pediatric Dentistry and Dental Public Health Department (Prof M El Tantawi PhD), Alexandria University, Alexandria, Egypt; Department of Clinical Pathology (Prof M El Sayed Zaki PhD), Mansoura University, Mansoura, Egypt; Department of Public Health (I Elbarazi DrPH), Abu Dhabi University, Khalifa City, United Arab Emirates; Department of Psychiatry (A C Tsai MD), Division of Cardiology (I Y Elgendy MD), Massachusetts General Hospital, Boston, MA, USA; Eijkman-Oxford Clinical Research
Unit (I R Elyazar PhD), Eijkman Institute for Molecular Biology, Jakarta, Indonesia; Psychiatric Epidemiology and Burden of Disease Research (A M Mantilla Herrera PhD), West Moreton Hospital Health Service (D F Santomauro PhD), Queensland Centre for Mental Health Research, Brisbane, QLD, Australia (H E Erskine PhD); Department of Health Management, Policy, and Economics (V Yazdi-Feyzabadi PhD), Department of Medicinal Chemistry (K Eskandari PhD), HIV/STI Surveillance Research Center, and WHO Collaborating Center for HIV Surveillance (Prof H Sharifi PhD, H Tohidinik PhD), Health Services Management Research Center (V Yazdi-Feyzabadi PhD), Pharmaceutics Research Center (K Eskandari PhD), Kerman University of Medical Sciences, Kerman, Iran; Department of Anatomical Sciences (I Halvaei PhD), Department of Clinical Biochemistry (A Mosapour PhD), Department of Parasitology and Entomology (Prof F Ghaffarifar PhD, L Zaki PhD), Department of Physiology (S Esmaeilnejad PhD), Tarbiat Modares University, Tehran, Iran; Department of Healthcare Management (E Hasanpoor PhD), Department of Microbiology (A Hasanzadeh PhD), Department of Nutrition and Food Sciences (H Pourjafar PhD), Department of Public Health (F Esmaeilzadeh PhD, M Khodayari PhD), Maragheh University of Medical Sciences, Maragheh, Iran; Division of Cancer Epidemiology and Genetics (A Etemadi PhD, Q Lan PhD), National Cancer Institute, Bethesda, MD, USA; School of Nursing (T Y Yeheyis MSc), Unit of Medical Physiology (A E Etisso MSc), Hawassa University, Hawassa, Ethiopia; Independent Consultant, Awka, Nigeria (O Ezekannagha PhD); International Institute for Tropical Agriculture, Ibadan, Nigeria (O Ezekannagha PhD); Dissemination Division (C S e Farinha MSc), National Institute of Statistics, Lisbon, Portugal; Activity Planning and Control Unit (C S e Farinha MSc), Directorate-General of Health (DGS), Lisbon, Portugal; Department of Medical and Surgical Sciences (A Farioli PhD, Prof F S Violante MD), University of Bologna, Bologna, Italy; Department of Biology and Biotechnology "Lazzaro Spallanzani" (P S Faris PhD), University of Pavia, Pavia, Italy; Department of Biology (P S Faris PhD), Department of Forestry (D K Mohammad PhD), Salahaddin University-Erbil, Erbil, Iraq; Department of Psychology (Prof A Faro PhD), Federal University of Sergipe, São Cristóvão, Brazil; Division of Neurology (S Fereshtehnejad PhD), School of Epidemiology and Public Health (H M Orpana PhD), School of International Development and Global Studies (Prof S Yaya PhD), University of Ottawa, Ottawa, ON, Canada; Division of Research (J O Lam PhD), Psychiatry Department (I Filip MD), Kaiser Permanente, Fontana, CA, USA; School of Health Sciences (I Filip MD), A.T. Still University, Mesa, AZ, USA; Institute of Gerontological Health Services and Nursing Research (F Fischer PhD), Ravensburg-Weingarten University of Applied Sciences, Weingarten, Germany; Sergio Arouca National School of Public Health, Rio de Janeiro, Brazil (L S Flor MPH); Federal University of Espírito Santo, Vitória, Brazil (L S Flor MPH); Institute of Gerontology (N A Foigt PhD), National Academy of Medical Sciences of Ukraine, Kyiv, Ukraine; Department of Child Dental Health (Prof M O Folayan FWACS), Obafemi Awolowo University, Ile-Ife, Nigeria; Department of Cell Biology and Biotechnology

(A A Fomenkov PhD), Timiryazev Institute of Plant Physiology, Moscow, Russia; Department of Global Pediatric Medicine (L M Force MD), St Jude Children's Research Hospital, Memphis, TN, USA; Abadan Faculty of Medical Sciences (M Foroutan PhD), Abadan School of Medical Sciences, Abadan, Iran; Department of Family Medicine and Primary Care (J M Francis PhD), University of the Witwatersrand, Johannesburg, South Africa; College of Public Health, Medical, and Veterinary Sciences (R C Franklin PhD, A E Peden PhD), James Cook University, Douglas, QLD, Australia; Department of Dermatology (T Fukumoto PhD), Kobe University, Kobe, Japan; Department of Cardiovascular Medicine (M M Gad MD), Cleveland Clinic, Cleveland, OH, USA; National Center for Disease Control and Public Health, Tbilisi, Georgia (Prof A Gamkrelidze PhD, M Kereselidze PhD); Department of Tuberculosis (A L Garcia-Basteiro PhD), Manhiça Health Research Center (CISM), Manhiça, Mozambique; Viral and Bacterial Infections Research Program (A L Garcia-Basteiro PhD), Barcelona Institute for Global Health, Barcelona, Spain (Prof J V Lazarus PhD, Prof K Straif PhD); College of Medicine and Public Health (H A Gesesew PhD), Flinders University, Adelaide, SA, Australia; Telethon Kids Institute, Perth, WA, Australia (Prof P W Gething PhD); 
Institute of Health Research (H Ghiasvand PhD), University of Exeter, Exeter, UK; Research Group for Genomic Epidemiology (N Ghith PhD), Technical University of Denmark, Copenhagen, Denmark; Department of Clinical Sciences (Prof B Norrving PhD), Lund University, Malmö, Sweden; Department of Epidemiology and Biostatistics (K M Mehta DSc), Department of Radiation Oncology (O Mohamad MD), Health Hub (J S Salama MSc), Institute for Global Health Sciences (R Ghosh PhD), University of California San Francisco, San Francisco, CA, USA; Department of Cardiovascular Endocrinemetabolic Diseases and Aging (S Giampaoli MD), Italian National Institute of Health, Rome, Italy; Faculty of Allied Health Sciences (Prof S Gilani PhD), University Institute of Public Health (S Hameed MPH, A Hanif PhD, Prof S M Rana PhD), The University of Lahore, Lahore, Pakistan; Afro-Asian Institute, Lahore, Pakistan (Prof S Gilani PhD); Division of Health Sciences (O A Uthman PhD), Medical School (Prof P S Gill DM), University of Warwick, Coventry, UK; Department of Community and Family Medicine (R F Gillum MD), Division of General Internal Medicine (R F Gillum MD), Howard University, Washington, DC, USA; Family Medicine Research Center (Prof I A Ginawi MD), Ministry of Health, Hail, Saudi Arabia; Discipline of Public Health Medicine (T G Ginindza PhD, B Tlou DrPH), University of KwaZulu-Natal, Durban, South Africa (T G Ginindza PhD, Prof F C Tanser PhD); Third Department of Neurology (E V Gnedovskaya PhD), Research Center of Neurology, Moscow, Russia (M A Kravchenko PhD, Prof M A Piradov DSc); Health Systems and Policy Research (M Golechha PhD), Indian Institute of Public Health Gandhinagar, Gandhinagar, India; Center for the Studies in Regional Development (CSRD) (S Goli PhD), Jawahar Lal Nehru University, New Delhi, India; Department of Computer Science and Software Engineering (Prof G J Milne PhD), Dobney Hypertension Centre (Prof M P Schlaich MD), Medical School (Prof G J Hankey MD), UWA Public Policy Institute (S Goli PhD), University of Western Australia, Perth, WA, Australia; Department of Exercise and Health Sciences (P N Gona PhD), University of Massachusetts Boston, Boston, MA, USA; Hudson College of Public Health (S V Gopalani MPH), University of Oklahoma Health Sciences Center, Oklahoma City, OK, USA; Department of Health and Social Affairs (S V Gopalani MPH), Government of the Federated States of Micronesia, Palikir, Federated States of Micronesia; Center for Environmental and Health Sciences (H Goudarzi PhD), Department of Respiratory Medicine (H Goudarzi PhD), Hokkaido University, Sapporo, Japan; Department of Computer Science (R Rawassizadeh PhD), Department of Dermatology (A Grada MD), Department of Global Health, School of Public Health (E O Nsoesie PhD), Health Informatic Lab (T Javaheri PhD), Boston University, Boston, MA, USA; Academic Public Health England (Prof S Rawaf MD), Health Improvement Directorate (F Greaves PhD), Public Health England, London, UK (Prof N Steel PhD); Department of Family and Community Medicine (M I M Gubari PhD), University of Sulaimani, Sulaimani, Iraq; Department of Epidemiology (Prof H C Gugnani PhD), Department of Microbiology (Prof H C Gugnani PhD), Saint James School of Medicine, The Valley, Anguilla; School of Dentistry (Prof A L S Guimaraes PhD), State University of Montes Claros, Montes Claros, Brazil; Institute of Tropical Pathology and Public Health (IPTSP) (R A Guimarães MSc), Federal University of Goias, Goiânia, Brazil; College of Medicine and Health Science (R A Guled PhD), Department of Food Science and Nutrition (A M Senbeta MSc), Department of Public Health (A Hashi PhD, M O Omer MSc, A Y Yousuf PhD), Jigjiga University, Jijiga, Ethiopia; Department of Epidemiology (Prof Y Guo PhD), Binzhou Medical University, Yantai City, China; Department of Community Medicine (Prof S S Gupta MD), Mahatma Gandhi Institute of Medical Sciences, Sevagram, India; Department of Civil Engineering (Prof T Gupta DSc), Indian Institute of Technology Kanpur, Kanpur, India; Department of Preventive Cardiology (Prof R Gupta MD), Eternal Heart Care Centre \& Research Institute, Jaipur, India; Department of Medicine (Prof R Gupta MD), Mahatma Gandhi University Medical Sciences, Jaipur, India; Medical Resources (Prof R Gupta MD), March of Dimes, Arlington, VA, USA; Health Policy, Management and Leadership (Prof R Gupta MD), West Virginia University School of Public Health, Morgantown, WV, USA; Department of Public Health (J A Haagsma PhD), Department of Public health (S Polinder PhD),
Erasmus University Medical Center, Rotterdam, Netherlands; Clinical Neurological Sciences (V Hachinski DSc), Department of Epidemiology \& Biostatistics (Prof S Stranges MD), The University of Western Ontario, London, ON, Canada; Lawson Health Research Institute, London, ON, Canada (V Hachinski DSc); College of Medicine (A Hafiz PhD), Umm AL Qura University, Makkah, Saudi Arabia; Department of Family and Community Medicine (Prof R R Hamadeh PhD), Arabian Gulf University, Manama, Bahrain; School of Health and Environmental Studies (Prof S Hamidi DrPH), Hamdan Bin Mohammed Smart University, Dubai, United Arab Emirates; Department of Neurology (Prof G J Hankey MD), Sir Charles Gairdner Hospital, Perth, WA, Australia; Carlos III Health Institute (Prof R Tabarés-Seisdedos PhD), Biomedical Research Networking Center for Mental Health Network (CiberSAM), Barcelona, Spain (J M Haro MD, S Tyrovolas PhD); Department of Zoology and Entomology (A I Hasaballah PhD), Al Azhar University, Cairo, Egypt; Biology Department (M Hashemian PhD), Utica College, Utica, NY, USA; Caspian Digestive Disease Research Center (S Hassanipour PhD, F Joukar PhD,

Prof F Mansour-Ghanaei MD), Department of Environmental Health Engineering (J Jaafari PhD), Gastrointestinal and Liver Diseases Research Center (S Hassanipour PhD, F Joukar PhD,

Prof F Mansour-Ghanaei MD), Guilan University of Medical Sciences, Rasht, Iran; Independent Consultant, Tabriz, Iran (H Hassankhani PhD); Skaane University Hospital (R J Havmoeller PhD), Skaane County Council, Malmoe, Sweden; International Foundation for Dermatology, London, UK (Prof R J Hay MD); Institute of Pharmaceutical Sciences (K Hayat MS), University of Veterinary and Animal Sciences, Lahore, Pakistan; Department of Pharmacy Administration and Clinical Pharmacy (K Hayat MS), Xian Jiaotong University, Xian, China; Center for Environmental and Respiratory Health Research (B Heibati PhD), University of Oulu, Oulu, Finland; Independent Consultant, Santa Clara, CA, USA (G Heidari MD); Department of Public Health (A Henok MPH), Mizan-Tepi University, Mizan Teferi, Ethiopia; School of Business (Prof C Herteliu PhD), London South Bank University, London, UK; Department for Health (T R Hird PhD), Department of Mathematical Sciences (P Moraga PhD), University of Bath, Bath, UK; Hypertension and Kidney Disease Laboratory (Prof M P Schlaich MD), Baker Heart and Diabetes Institute, Melbourne, VIC, Australia; Department of Epidemiology (Prof H W Hoek MD), Department of Health and Behavior Studies (Prof I D Sigfusdottir PhD), Department of Psychiatry (Prof M R Phillips MD), Columbia University, New York, NY, USA; Department of Pediatrics (M K Hole MD), University of Texas Austin, Austin, TX, USA; Department of Health Economics, Lancaster University, Lancashire, UK (Prof B Hollingsworth PhD); Metrics and Evaluation (K P Hopf MPH), Operation Smile, Virginia Beach, VA, USA; Independent Consultant, Virginia Beach, VA, USA (K P Hopf MPH); Department of Pulmonology (N Horita PhD), Yokohama City University, Yokohama, Japan; Department of Epidemiology and Population Health (H Hosgood PhD), Albert Einstein College of Medicine, Bronx, NY, USA; Department of Pharmacology (N Hossain MPhil), Bangladesh Industrial Gases Limited, Tangail, Bangladesh; Institute for Global Health Innovations (H T Mai MPH, C T Nguyen MPH, D N Nguyen MA, H L T Nguyen MPH, H Q Pham MD), Institute of Research and Development (M Hosseinzadeh PhD), Faculty of Pharmacy (D N Nguyen MA), Duy Tan University, Da Nang, Vietnam; Clinical Legal Medicine Department (S Hostiuc PhD), National Institute of Legal Medicine Mina Minovici, Bucharest, Romania; Division of Information and Computing Technology (Prof M Househ PhD), Hamad Bin Khalifa University, Doha, Qatar; Faculty of Medicine of Tunis (Prof M Hsairi MPH), University Tunis El Manar, Tunis, Tunisia; College of Public Health (R Lin PhD), Department of Health Services Administration (V Hsieh PhD), Department of Occupational Safety and Health (Prof B Hwang PhD), China Medical University, Taichung, Taiwan; School of Public Health (K Hu PhD), Zhejiang University, Hangzhou, China; Maternal and Child Health Division (T M Huda PhD, S Zaman MPH), International Centre for Diarrhoeal Disease Research, Bangladesh, Dhaka, Bangladesh; Department of Public Health and Community Medicine (Prof A Humayun PhD), Shaikh Khalifa Bin Zayed Al-Nahyan Medical College, Lahore, Pakistan; School of Pharmaceutical Sciences (R Hussain PhD), University of Science 
Malaysia, Penang, Malaysia; Nigerian Field Epidemiology and Laboratory Training Program (C U Ibeneme MPH), African Field Epidemiology Network, Abuja, Nigeria; Department of Public Health and Disease Control (C U Ibeneme MPH), Ministry of Health, Umuahia, Nigeria; International Center for Nutrition and Information (N Ikeda PhD), National Institutes of Biomedical Innovation, Health and Nutrition, Tokyo, Japan; Department of Epidemiology (Prof M D Ilic PhD), Department of Global Health, Economics and Policy (Prof M Jakovljevic PhD), University of Kragujevac, Kragujevac, Serbia; Division of Community Health and Family Medicine (L R Inbaraj MD), Bangalore Baptist Hospital, Bangalore, India; College of Public Health (U Iqbal PhD), Graduate Institute of Biomedical Informatics (M Islam MSc), Taipei Medical University, Taipei, Taiwan; Surveillance and Health Services Research (F Islami PhD), American Cancer Society, Atlanta, GA, USA; Graduate School of Medicine (Prof K Yamagishi MD), Department of Social Medicine (Prof H Iso MD), Osaka University, Suita-city, Japan; Department of Biochemistry and Microbiology (C C D Iwu MSc), University of Fort Hare, Alice, South Africa; Knowledge Translation (I O Iyamu MD), Centre for Health Evaluation and Outcome Sciences, Vancouver, BC, Canada; Department of Global and Community Health (K H Jacobsen PhD), George Mason University, Fairfax, VA, USA; Postgraduate Institute of Medicine (A U Jayatilleke PhD), University of Colombo, Colombo, Sri Lanka; Faculty of Graduate Studies (A U Jayatilleke PhD), Institute for Violence and Injury Prevention, Colombo, Sri Lanka; Achutha Menon Centre for Health Science Studies (P Jeemon PhD), Sree Chitra Tirunal Institute for Medical Sciences and Technology, Trivandrum, India; Department of Community Medicine (R P Jha MSc), Baba Saheb Ambedkar Medical College \& Hospital, Delhi, India; Department of Community Medicine (R P Jha MSc), Banaras Hindu University, Varanasi, India; Environmental Research Center (J S Ji DSc), Global Health Research Center (C Wu PhD), Duke Kunshan University, Kunshan, China; Department of Earth Observation Science (P Jia PhD), Faculty of Geo-Information Science and Earth Observation (F B Osei PhD), University of Twente, Enschede, Netherlands; Renal and Cardiovascular Division (O John MD), Research Division (P K Maulik PhD), University of New South Wales (Prof A E Schutte PhD), The George Institute for Global Health, Sydney, Australia; Independent Consultant, Eiksmarka, Norway (L Johansson DrPH); Beijing Institute of Ophthalmology (Prof J B Jonas MD), Beijing Tongren Hospital, Beijing, China; Faculty of Health and Public Administration (M Szócska PhD), Health Services Management Training Centre (T Joo MSc), Semmelweis University, Budapest, Hungary; Department of Family Medicine and Public Health (J J Jozwiak PhD), University of Opole, Opole, Poland; Institute of Family Medicine and Public Health (M Jürisson PhD), University of Tartu, Tartu, Estonia; School of Public Health (Z Kabir PhD), University College Cork, Cork, Ireland; Golestan Research Center of Gastroenterology and Hepatology (GRCGH) (G Roshandel PhD), Gorgan Congenital Malformations Research Center (M Oladnabi PhD), Infectious Diseases Research Center (H Kalani PhD), Golestan University of Medical Sciences, Gorgan, Iran; Centre for Therapeutic Target Validation (M A Karim MD), Wellcome Trust Sanger Institute, Cambridge, UK; Department of Health Management and System Sciences (S Karimi PhD), University of Louisville, Louisville, KY, USA; Center for Health Equity (S Karimi PhD), Louisville Metro Department of Public Health \& Wellness, Louisville, KY, USA; College of Health Sciences (G M Kassa MSc), Debre Markos University, Debre Markos, Ethiopia; MRC/CSO Social and Public Health Sciences Unit (S V Katikireddi PhD), University of Glasgow, Glasgow, UK; Department of Diabetes and Metabolic Diseases (T Yamada MD), Department of Global Health Policy (S Nomura PhD), Department of Mental Health (Prof N Kawakami PhD), University of Tokyo, Tokyo, Japan; International Research Center of Excellence (G A Kayode PhD), Institute of Human Virology Nigeria, Abuja, Nigeria; Institute for Risk Assessment Sciences (IRAS) (Prof H Kromhout PhD, E Traini MSc), Julius Centre for Health Sciences and Primary Care (G A Kayode PhD), Utrecht University, Utrecht, Netherlands; Department of Psychiatry (M Kumar PhD), Open, Distance and eLearning Campus (Prof P N Keiyoro PhD), School of Economics (M K Muriithi PhD), School of Public Health (R G Wamai PhD), University of Nairobi, Nairobi, Kenya; Department of Public Health (Prof Y S Khader PhD),
Jordan University of Science and Technology, Irbid, Jordan; School of Food and Agricultural Sciences (N Khalid PhD), University of Management and Technology, Lahore, Pakistan; Department of Epidemiology and Biostatistics (E A Khan MPH), Health Services Academy, Islamabad, Pakistan; Department of Health Policy and Management (Prof Y Khang MD), Institute of Health Policy and Management (Prof Y Khang MD), Seoul National University, Seoul, South Korea; Faculty of Health and Wellbeing (K Khatab PhD), Sheffield Hallam University, Sheffield, UK; College of Arts and Sciences (K Khatab PhD), Ohio University, Zanesville, OH, USA; The Iranian Academy of Medical Sciences, Tehran, Iran (M Khayamzadeh MD); Department of Nutrition and Health Science (Prof J Khubchandani PhD), Ball State University, Muncie, IN, USA; Division of Child \& Adolescent Psychiatry (C Kieling MD), Clinical Hospital, Porto Alegre, Brazil; Department of Preventive Medicine (Y Kim PhD, Prof S Yoon PhD), Korea University, Seoul, South Korea (Prof M Shin PhD); Korea Health Industry Development Institute, Cheongju-si, South Korea (C Kim PhD); School of Traditional Chinese Medicine (Y Kim PhD), Xiamen University Malaysia, Sepang, Malaysia; Cultures, Societies and Global Studies, \& Integrated Initiative for Global Health (R G Wamai PhD), Department of Health Sciences (Prof D Kim DrPH), Northeastern University, Boston, MA, USA Department of Nutrition (R W Kimokoti MD), Simmons University, Boston, MA, USA; Faculty of Health (Y Kinfu PhD), University of Canberra, Canberra, ACT, Australia; College of Medicine (Y Kinfu PhD), Qatar University, Doha, Qatar; Department of Nursing and Health Promotion (S Kisa PhD), Oslo Metropolitan University, Oslo, Norway; School of Health Sciences (Prof A Kisa PhD), Kristiania University College, Oslo, Norway; Global Community Health and Behavioral Sciences (Prof A Kisa PhD), Tulane University, New Orleans, LA, USA; Department of Health Economics and Social Security (K Kissimova-Skarbek PhD), Institute of Public Health (R Topor-Madry PhD), Jagiellonian University Medical College, Krakow, Poland; Department of Public Health (Prof M Kivimäki PhD,

Prof T Lallukka PhD), University of Helsinki, Helsinki, Finland (T J Meretoja MD); Faculty of Dental Sciences

(Prof K M Shivakumar PhD), Krishna Institute of Medical Sciences, Karad, India; Centre for Disease Burden (A S Knudsen PhD), Division of Mental and Physical Health (Prof S Øverland PhD), Norwegian Institute of Public Health, Bergen, Norway (G Sulo PhD); Public Health Sciences Division (J M Kocarnik PhD), Fred Hutchinson Cancer Research Center, Seattle, WA, USA; Global Healthcare Consulting, New Delhi, India (S Kochhar MD); Institute of Health Sciences (Prof D S Q Koh PhD), Gadong, Brunei; Brain Engineering Research Center (H Komaki MD), Institute for Research in Fundamental Sciences, Tehran, Iran; Arthritis Research Canada, Richmond, BC, Canada (J A Kopec PhD); Independent Consultant, Jakarta, Indonesia (S Kosen MD); Department of Global Health (A Kotlo MS), Vancouver Virology Centre, Vancouver, BC, Canada; Department of Internal and Pulmonary Medicine (Prof P A Koul MD), Sheri Kashmir Institute of Medical Sciences, Srinagar, India; Biomedical Research Networking Center for Mental Health Network (CIBERSAM) (A Koyanagi MD), St. John of God Health Park (S Tyrovolas PhD), San Juan de Dios Sanitary Park, Sant Boi de Llobregat, Spain; Department of Anthropology (K Krishan PhD), Panjab University, Chandigarh, India; Department of Psychiatry

(Prof S KS MD), Government Medical College Thrissur, Thrissur, India; Kerala University of Health Scinces, Thrissur, India (Prof S KS MD); Department of Demography (Prof B Kuate Defo PhD), Department of Social and Preventive Medicine (Prof B Kuate Defo PhD), University of Montreal, Montreal, QC, Canada; Department of Medical Education and Informatics (B Kucuk Bicer PhD), Gazi University, Ankara, Turkey; Cancer Institute (D Yuce MD), Hacettepe University, Ankara, Turkey; Department of Mathematical Demography \& Statistics (R Rawat MPhil), Department of Public Health and Mortality Studies (W B Meitei MSc), International Institute for Population Sciences, Mumbai, India (P Kumar PhD); Department of Humanities and Social Sciences (P Singh MSc), Mechanical and Industrial Engineering (G Kumaresh MS), Indian Institute of Technology, Roorkee, Roorkee, India; Faculty of Public Health (D Kusuma DSc), University of Indonesia, Depok, Indonesia; National Institute for Health Research (NIHR) Oxford Biomedical Research Centre, Oxford, UK (B Lacey PhD); 
Department of Community and Family Medicine (F H Lami PhD), University of Baghdad, Baghdad, Iraq; Unit of Genetics and Public Health (Prof I Landires MD), Unit of Microbiology and Public Health (V Nunez-Samudio PhD), Institute of Medical Sciences, Las Tablas, Panama; Department of Public Health (V Nunez-Samudio PhD), Ministry of Health, Herrera, Panama (Prof Landires MD); Medical Director (Prof V C Lansingh PhD), HelpMeSee, New York, NY, USA; General Director (Prof V C Lansingh PhD), Mexican Institute of Ophthalmology, Queretaro, Mexico; Belo Horizonte City Hall (Prof S Lansky PhD), Municipal Health Department of Belo Horizonte, Belo Horizonte, Brazil; Department of Medical Sciences (Prof A O Larsson PhD), Uppsala University, Uppsala, Sweden; Department of Clinical Chemistry and Pharmacology (Prof A O Larsson PhD), Uppsala University Hospital, Uppsala, Sweden; Department of Otorhinolaryngology (S Lasrado MS), Father Muller Medical College, Mangalore, India; Department of Neurology and Psychiatry (P M Lavados MD), German Clinic of Santiago, Santiago, Chile; Faculty of Medicine (P M Lavados MD), Univeristy of Development, Santiago, Chile; Centre for Clinical Epidemiology (L F Leal PhD), Jewish General Hospital, Montreal, QC, Canada; College of Optometry (J L Leasher OD), Nova Southeastern University, Fort Lauderdale, FL, USA; School of Pharmacy (S W H Lee PhD), Taylor's University Lakeside Campus, Subang Jaya, Malaysia; Neurology, Public Health and Disability Unit (M Leonardi MD, A Raggi PhD, D Sattin PsyD, S Schiavolin MSc), Carlo Besta Neurological Institute IRCCS, Milan, Italy; Department of Sociology (B Li PhD), Shenzhen University, Shenzhen, China; Department of Medicine (L Lim MRCP), University of Malaya, Kuala Lumpur, Malaysia; Department of Medicine and Therapeutics (L Lim MRCP), The Chinese University of Hong Kong, Shatin, China; Asbestos Diseases Research Institute, Concord, NSW, Australia (R Lin PhD, Prof K Takahashi PhD); UCD Centre for Disability Studies (C Linehan PhD), University College Dublin, Dublin, Ireland; School of Public Health (Prof S Linn DrPH, K Shuval PhD), University of Haifa, Haifa, Israel; Department of Dentistry- Quality and Safety of Oral Health Care (Prof S Listl PhD), Radboud University, Nijmegen, Netherlands; Department of Translational Health Economics (Prof S Listl PhD), Heidelberg University Hospital, Heidelberg, Germany; National Center for Chronic and Noncommunicable Disease Control and Prevention (M Zhou PhD), Chinese Center for Disease Control and Prevention, Beijing, China (Prof S Liu PhD); Department of Epidemiology (S Liu MD), Brown University, Providence, RI, USA; Department of Basic Medical Sciences, Neuroscience and Sense Organs (Prof G Logroscino PhD), University of Bari Aldo Moro, Bari, Italy; Department Clinical Research in Neurology (Prof G Logroscino PhD), Fondazione Cardinale Giovanni Panico Hospital, Tricase, Italy; Population Health Sciences (K J Looker PhD), University of Bristol, Bristol, UK; Alliance for Improving Health Outcomes, Inc., Quezon City, Philippines (J F Lopez MD); Institute of Nutritional Sciences (Prof S Lorkowski PhD), Friedrich Schiller University Jena, Jena, Germany; Competence Cluster for Nutrition and Cardiovascular Health (nutriCARD), Jena, Germany (Prof S Lorkowski PhD); Department of General Surgery (Prof R Lunevicius DSc), Liverpool University Hospitals NHS Foundation Trust, Liverpool, UK; Department of Surgery (Prof R Lunevicius DSc), Institute of Population Health Sciences (M R Mathur PhD), University of Liverpool, Liverpool, UK; School of Medicine (Prof R A Lyons MD), Swansea University, Swansea, UK; Center for Integration of Data and Health Knowledge (D B Machado PhD), Oswald Cruz Foundation (FIOCRUZ), Salvador, Brazil; Value-based Healthcare (F Madotto PhD), IRCCS MultiMedica, Sesto San Giovanni, Italy; Radiology Department

(H Magdy Abd El Razek MD), Mansoura Faculty of Medicine, Mansoura, Egypt; Ophthalmology Department (M Magdy Abd El Razek MSc), Ministry of Health \& Population, Aswan, Egypt; Academy of Medical Science, (M M Mahdavi PhD); Radiology and Precision Health Program (M Mahmoudi PhD), Michigan State University, East Lansing, MI, USA; Faculty of Medicine (J N Malagón-Rojas MSc), El Bosque University, Bogota, Colombia; Department of Forensic Medicine (Prof V Maled MD), Rajiv Gandhi University of Health Sciences, Dharwad, India; Department of Forensic Medicine (Prof V Maled MD), Shri Dharmasthala Manjunatheshwara University, Dharwad, India; Department of Epidemiology and Biostatistics (G Moradi PhD),
Environmental Health Research Center (Prof A Maleki PhD), Social Determinants of Health Research Center (G Moradi PhD), Kurdistan University of Medical Sciences, Sanandaj, Iran; Department of Surgery (A Manafi MD), University of Virginia, Charlottesville, VA, USA; General Surgery Department I (A Manda MD), Emergency University Hospital Bucharest, Bucharest, Romania; Department of Population Studies (C Mapoma PhD), University of Zambia, Lusaka, Zambia; Department of Energy, Environmental, and Chemical Engineering (Prof R V Martin PhD), Washington University, St Louis, NS, USA; Faculty of Public Health (S Martini PhD), Airlangga University, Surabaya, Indonesia; Indonesian Public Health Association, Surabaya, Indonesia (S Martini PhD); Campus Caucaia (F R Martins-Melo PhD), Federal Institute of Education, Science and Technology of Ceará, Caucaia, Brazil; Faculty of Health and Education (A Masaka MSc), Botho University-Botswana, Gaborone, Botswana; Department of Neurology (J Massano MD), University Hospital Center de Sao Joao, Porto, Portugal; Department of Ophthalmology (C McAlinden PhD), Singleton Hospital, Swansea, UK; National Centre for Register-based Research (Prof J J McGrath MD, N C Momen PhD, O Plana-Ripoll PhD), Aarhus University, Aarhus, Denmark; Academic Area of Dentistry (C E Medina-Solís MSc), Autonomous University of Hidalgo State, Pachuca, Mexico; Department of Nursing (A B Wondmieneh MSc), Department of Pharmacy (B Meharie MSc), Wollo University, Dessie, Ethiopia; Neurology Department (Prof M Mehndiratta MD), Janakpuri Super Specialty Hospital Society, New Delhi, India; Department of Neurology (Prof M Mehndiratta MD), Govind Ballabh Institute of Medical Education and Research, New Delhi, India; India Cancer Research Consortium (Prof R Mehrotra D.Phil), National Institute of Epidemiology (R Suliankatchi Abdulkader MD), Indian Council of Medical Research, New Delhi, India; Department of Nutrition (T Mekonnen MPH), University of Oslo, Oslo, Norway; Institute of Human Virology (P T N Memiah DrPH), School of Medicine (M T Wallin MD), University of Maryland, Baltimore, MD, USA; College of Medicine (Prof Z A Memish MD), Alfaisal University, Riyadh, Saudi Arabia; Research \& Innovation Center (Prof Z A Memish MD), Ministry of Health, Riyadh, Saudi Arabia; Peru Country Office (W Mendoza MD), United Nations Population Fund (UNFPA), Lima, Peru; Department of Cardiovascular Surgery (A Mereke MD), Asfendiyarov Kazakh National Medical University, Almaty, Kazakhstan; Breast Surgery Unit (T J Meretoja MD), Neurology Unit (A Meretoja MD), Helsinki University Hospital, Helsinki, Finland; Clinical Microbiology and Parasitology Unit (T Mestrovic PhD), Zora Profozic Polyclinic, Zagreb, Croatia; University Centre Varazdin (T Mestrovic PhD), University North, Varazdin, Croatia; Center for Innovation in Medical Education (B Miazgowski MD), Department of Propedeutics of Internal Diseases \& Arterial Hypertension (Prof T Miazgowski MD), Pomeranian Medical University, Szczecin, Poland (B Miazgowski MD); Department of Women-Mother-Child (I Michalek PhD), Vaud University Hospital Center, Lausanne, Switzerland; Pacific Institute for Research \& Evaluation, Calverton, MD, USA (T R Miller PhD); Global Institute of Public Health

(Prof G Mini PhD), Ananthapuri Hospitals and Research Institute, Trivandrum, India; Women's Institute for Social and Health Studies, Trivandrum, India (Prof G Mini PhD); Department of Environmental Health (M Miri PhD), Non-Communicable Diseases Research Centre (M Miri PhD), Sabzevar University of Medical Sciences, Sabzevar, Iran; Internal Medicine Programme (Prof E M Mirrakhimov PhD), Kyrgyz State Medical Academy, Bishkek, Kyrgyzstan; Department of Atherosclerosis and Coronary Heart Disease

(Prof E M Mirrakhimov PhD), National Center of Cardiology and Internal Disease, Bishkek, Kyrgyzstan; Research Center for Biochemistry and Nutrition in Metabolic Diseases (H Mirzaei PhD), Kashan University of Medical Sciences, Kashan, Iran; Institute of Addiction Research (ISFF) (B Moazen MSc), Frankfurt University of Applied Sciences, Frankfurt, Germany; Department of Epidemiology and Biostatistics (A Mohammadian-Hafshejani PhD), Shahrekord University of Medical Sciences, Shahrekord, Iran; Kashmar Center of Higher Health Education (R Mohammadpourhodki MSc), Neurogenic Inflammation Research Center (A Sahebkar PhD), Mashhad University of Medical Sciences, Mashhad, Iran; Department of Public Health (H Mohammed MPH), Dire Dawa University, Dire Dawa, Ethiopia; 
Department of Community Medicine (M B Sufiyan MD), Health Systems and Policy Research Unit (S Mohammed PhD), Ahmadu Bello University, Zaria, Nigeria; Pediatric Neurorehabilitation Research Center (Prof M A Mohseni Bandpei PhD), University of Social Welfare and Rehabilitation Sciences, Tehran, Iran; Clinical Epidemiology and Public Health Research Unit (L Monasta DSc, L Ronfani PhD), Burlo Garofolo Institute for Maternal and Child Health, Trieste, Italy; Department of Biomedical and Dental Sciences and Morphofunctional Imaging (Prof S Mondello MD), Messina University, Messina, Italy; National Center for Health Insurance Research (M Moradi-Joo PhD), Iran Health Insurance Organization, Tehran, Iran; International Laboratory for Air Quality and Health (Prof L Morawska PhD), Queensland University of Technology, Brisbane, QLD, Australia; Department of Food Science (Prof A Mousavi Khaneghah PhD), University of Campinas (Unicamp), Campinas, Brazil; Competence Center of Mortality-Follow-Up of the German National Cohort (R Westerman DSc), Demographic Change and Aging Research Area (A Werdecker PhD), Federal Institute for Population Research, Wiesbaden, Germany (Prof U O Mueller MD); Center for Population and Health, Wiesbaden, Germany

(Prof U O Mueller MD); Department of Endocrinology \& Metabolism (Prof S Mukhopadhyay MD), Seth Sukhlal Karnani Memorial Hospital, Kolkata, India; School of Medical Sciences (K Musa PhD), Science University of Malaysia, Kubang Kerian, Malaysia; Department of Pediatric Medicine (Prof G Mustafa MD), The Children's Hospital \& The Institute of Child Health, Multan, Pakistan; Department of Pediatrics \& Pediatric Pulmonology (Prof G Mustafa MD), Institute of Mother \& Child Care, Multan, Pakistan; Knowledge Translation and Utilization Unit (Prof A F Nabhan PhD), Egyptian Center for Evidence Based Medicine, Egypt; Research and Analytics Department (A J Nagarajan M.Tech), Initiative for Financing Health and Human Development, Chennai, India; Department of Research and Analytics (A J Nagarajan M.Tech), Bioinsilico Technologies, Chennai, India; Institute of Epidemiology and Medical Biometry (Prof G Nagel PhD, Prof D Rothenbacher MD), Ulm University, Ulm, Germany; Comprehensive Cancer Center (G Naik MPH), Department of Psychology (D C Schwebel PhD), School of Medicine

(Prof J A Singh MD), University of Alabama at Birmingham, Birmingham, AL, USA; Laboratory of Public Health Indicators Analysis and Health Digitalization (M Naimzada MD, N Otstavnov BA, S S Otstavnov PhD, S Soshnikov PhD, M V Titova PhD), Moscow Institute of Physics and Technology, Dolgoprudny, Russia; Experimental Surgery and Oncology Laboratory (M Naimzada MD), Kursk State Medical University, Kursk, Russia; Department of Pulmonary Medicine (S Nair MD), Government Medical College Trivandrum, Trivandrum, India; Health Action by People, Trivandrum, India (S Nair MD); Department of Dermatology (Prof L Naldi MD), San Bortolo Hospital, Vicenza, Italy; Study Center of the Italian Group for Epidemiologic Research in Dermatology (GISED) Study Center, Bergamo, Italy (Prof L Naldi MD); Suraj Eye Institute, Nagpur, India (V Nangia MD); Department for the Control of Disease, Epidemics, and Pandemics (J Nansseu MD), Ministry of Public Health, Yaoundé, Cameroon; Department of Internal Medicine and Specialties (Prof E Sobngwi PhD), Department of Public Health (G Nguefack-Tsague PhD, J Nansseu MD), University of Yaoundé I, Yaoundé, Cameroon; Disease Control and Environmental Health (R Ndejjo MSc), Makerere University, Kampala, Uganda; Department of General Surgery (I Negoi PhD), Emergency Hospital of Bucharest, Bucharest, Romania; Cardio-Aid, Bucharest, Romania (R I Negoi PhD); Research \& Statistics Unit (K N Ngari MSc), Synotech Consultants, Nairobi, Kenya; Department of Biological Sciences (J W Ngunjiri DrPH), University of Embu, Embu, Kenya; Institute for Mental Health Policy Research (K D Shield PhD, Y T Nigatu PhD), Centre for Addiction and Mental Health, Toronto, ON, Canada; Department of Clinical Epidemiology (Y T Nigatu PhD), Institute for Clinical Evaluative Sciences, Ottowa, ON, Canada; Department of Health Policy and Management (S Nomura PhD), Keio University, Tokyo, Japan; Administrative and Economic Sciences Department (Prof B Oancea PhD), University of Bucharest, Bucharest, Romania; Department of Preventive Medicine (I Oh PhD), Kyung Hee University, Dongdaemun-gu, South Korea; Disease Surveillance and Epidemic Response (E W Okunga MSc), Ministry of Health, Nairobi, Kenya; Department of Psychiatry (A T Olagunju MD), University of
Lagos, Lagos, Nigeria; Centre for Healthy Start Initiative, Lagos, Nigeria (J O Olusanya MBA, B O Olusanya PhD); Early Detection and Cancer Prevention Services (S Ong FAMS), Pantai Jerudong Specialist Centre, Bandar Seri Begawan, Brunei; Non-communicable Disease Prevention Unit (S Ong FAMS), Ministry of Health, Bandar Seri Begawan, Brunei; Department of Pharmacology and Therapeutics

(Prof O E Onwujekwe PhD), University of Nigeria Nsukka, Enugu, Nigeria; Graduate School of Public Health (Prof E Oren PhD), San Diego State University, San Diego, CA, USA; Health Systems Research Center (D V Ortega-Altamirano DrPH), National Public Health Institute, Cuernavaca, Mexico; Department of Medicine (Prof A Ortiz MD), Princess University Hospital (Prof J B Soriano MD), Autonomous University of Madrid, Madrid, Spain; Department of Nephrology and Hypertension (Prof A Ortiz MD), The Institute for Health Research Foundation Jiménez Díaz University Hospital, Madrid, Spain; Department of Environmental Management and Toxicology (O Osarenotor MSc), University of Benin, Benin City, Nigeria; Department of Mathematics and Statistics (F B Osei PhD), University of Energy and Natural Resources, Sunyani, Ghana; Biomedical Sciences (Prof S M Ostojic PhD), University of Novi Sad, Novi Sad, Serbia; Department of Health Care Administration and Economics (Prof V Vlassov MD), Department of Project Management (S S Otstavnov PhD), National Research University Higher School of Economics, Moscow, Russia; Department of Respiratory Medicine (Prof M P A DNB), Jagadguru Sri Shivarathreeswara Academy of Health Education and Research, Mysore, India; Department of Health Metrics (A Pana MD), Center for Health Outcomes \& Evaluation, Bucharest, Romania; National Institute of Health Research and Development (H U Pangaribuan MSc), Ministry of Health, Jakarta, Indonesia; Department of Medical Humanities and Social Medicine (Prof E Park PhD), Kosin University, Busan, South Korea; Division of General Internal Medicine (D Pasupula MD), University of Pittsburgh Medical Center, Pittsburgh, PA, USA; Department of Epidemiology (J R Patel PhD), University of Arkansas for Medical Sciences, Little Rock, AR, USA; Department of Poverty, Gender and Youth (S K Patel PhD), Population Council, New Delhi, India; School of Medicine

(A J Paternina-Caicedo MSc), University of Sinu, Cartagena, Colombia; Research \& Development Department (M Pathak PhD), Kalinga Institute of Medical Sciences, Bhubaneswar, India; Department of Pediatrics (Prof A Pathak PhD), RD Gardi Medical College, Ujjain, India; Community Health Sciences Department (Prof S B Patten PhD), Department of Medicine (Prof M Tonelli MD), University of Calgary, Calgary, AB, Canada; Centre for Adolescent Health

(Prof S M Sawyer MD), Critical Care and Neurosciences Department (Prof R G Weintraub MB), Population Health Theme (Prof G C Patton MD), Murdoch Childrens Research Institute, Melbourne, VIC, Australia; Public Health Youth Society of Nepal, Pokhara, Nepal (S Paudel MPH); Public Health Update, Pokhara, Nepal (S Paudel MPH); Center for Research and Innovation (V F Pepito MSc), Ateneo De Manila University, Pasig City, Philippines; Department of Environmental Medicine (Prof G D Thurston ScD), School of Global Public Health (E K Peprah PhD), New York University, New York, NY, USA; Department of Orthopedics (J Pereira MS), Yenepoya Medical College, Mangalore, India; Center for Integration of Data and Health Knowledge (J M Pescarini PhD), Oswaldo Cruz Foundation, Salvador, Brazil; Shanghai Mental Health Center (Prof M R Phillips MD), Shanghai Jiao Tong University, Shanghai, China; SSD Epidemiology Screening (C Piccinelli MS), City of Health and Science University Hospital of Turin, Turin, Italy; Department of Cardiology (T Pilgrim MD), University of Bern, Bern, Switzerland; Department of Environmental Hygiene (D Plass DrPH), German Environment Agency, Dessau-Roßlau, Germany; HIV and Mental Health Department (K N Pokhrel PhD), Integrated Development Foundation Nepal, Kathmandu, Nepal; Clinical Research Center (S I Prada PhD), Valle del Lili Foundation; Center for Studies in Social Protection and Health Economics (S I Prada PhD), ICESI University, Cali, Colombia; Askok \& Rita Patel Institute of Physiotherapy (V Prakash PhD), Charotar University of Science and Technology, Anand, India; Department of Neurology (Prof S Prakash DM), Smt. B.K.S. Medical Institute and Research Center, Vadodara, India; Department of Nephrology (Prof N Prasad DM), Sanjay Gandhi Postgraduate Institute of Medical 
Sciences, Lucknow, India; National Institute of Infectious Diseases, Bucuresti, Romania (L Preotescu PhD, M Shigematsu PhD); Health Sciences Department (D R A Pribadi MSc), Nursing Department (A Sudaryanto MPH), Muhammadiyah University of Surakarta, Sukoharjo, Indonesia; Biomedical Engineering Department (Prof M Rabiee PhD), Amirkabir University of Technology, Tehran, Iran; College of Medicine (A Radfar MD), University of Central Florida, Orlando, FL, USA; Department of Community Medicine (M Rahman PhD), Maharishi Markandeshwar Institute of Medical Sciences \& Research, Ambala, India; School of Nursing and Healthcare Professions (M Rahman PhD), Federation University Australia, Berwick, VIC, Australia; Public Health Department (Prof S M Rana PhD), University of Health Sciences, Lahore, Pakistan; Research Department (C L Ranabhat PhD), Policy Research Institute, Kathmandu, Nepal; Health and Public Policy Department (C L Ranabhat PhD), Global Center for Research and Development, Kathmandu, Nepal; Department of Medicine (A Ranta PhD), School of Physiotherapy (D C Ribeiro PhD), University of Otago, Wellington, New Zealand; Department of Neurology (A Ranta PhD), Capital \& Coast District Health Board, Wellington, New Zealand; Department of Oral Pathology (S Rao MDS), Srinivas Institute of Dental Sciences, Mangalore, India; University College London Hospitals, London, UK (D L Rawaf MD); School of Health, Medical and Applied Sciences (L Rawal PhD), CQ University, Sydney, NSW, Australia; School of Nursing and Midwifery (V Renjith PhD), Royal College of Surgeons in Ireland - Bahrain, Muharraq Governorate, Bahrain; Brien Holden Vision Institute, Sydney, NSW, Australia (Prof S Resnikoff MD); Network of Immunity in Infection, Malignancy and Autoimmunity (NIIMA) (Prof N Rezaei PhD) Universal Scientific Education and Research Network (USERN), Tehran, Iran; Department of Surgery (J Rickard MD), University of Minnesota, Minneapolis, MN, USA; Department of Surgery (J Rickard MD), University Teaching Hospital of Kigali, Kigali, Rwanda; Department of Clinical Research (L Roever PhD), Federal University of Uberlândia, Uberlândia, Brazil; Department of Neuroscience (M Romoli MD), University of Perugia, Perugia, Italy; Department of Neurology (M Romoli MD), Rimini “Infermi” Hospital - AUSL Romagna, Rimini, Italy; Centre for Social Research on Alcohol and Drugs (Prof R Room PhD), Stockholm University, Stockholm, Sweden; School of Medicine (M Rostamian PhD), Gonabad University of Medical Sciences, Gonabad, Iran; Agrosavia, Palmira, Colombia (E Rubagotti PhD); Department of Health Statistics (S F Rumisha PhD), National Institute for Medical Research, Dar es Salaam, Tanzania; Department of Internal Medicine (G M Rwegerera MD), University of Botswana, Gaborone, Botswana; Neuropsychiatric Institute (Prof P S Sachdev MD), Prince of Wales Hospital, Randwick, NSW, Australia; Halal Research Center of IRI (A Sahebkar PhD), Food and Drug Administration of the Islamic Republic of Iran, Tehran, Iran; Institute of Soil and Environmental Sciences (S Ullah PhD), University of Agriculture, Faisalabad, Faisalabad, Pakistan; Department of Phytochemistry (Prof S Sajadi PhD), Soran University, Soran, Iraq; Department of Nutrition (Prof S Sajadi PhD), Cihan University-Erbil, Kurdistan Region, Iraq; Department of Microbiology (N Salam PhD), Central University of Punjab, Bathinda, India; Research Department (S Salehi Zahabi PhD), Taleghani Hospital, Kermanshah, Iran; Global Health Institute (O M Salman MSc), American University of Beirut, Beirut, Lebanon; Center for Health Policy \& Center for Primary Care and Outcomes Research (Prof J A Salomon PhD), Stanford University, Stanford, CA, USA; Health and Disability Intelligence Group (I Salz MD), Ministry of Health, Wellington, New Zealand; Department of Surgery (Prof J Sanabria MD), Marshall University, Huntington, WV, USA; Public Health Unit, ACES Grande Porto VIII - Espinho/Gaia (J V Santos MD), Northern Region Health Administration, Vila Nova de Gaia, Portugal; Department of Community Medicine

(S Y Saraswathy PhD), PSG Institute of Medical Sciences and Research, Coimbatore, India; PSG-FAIMER South Asia Regional Institute, Coimbatore, India (S Y Saraswathy PhD); Faculty of Medicine (Prof R Sarmiento-Suárez MPH), University of Applied and Environmental Sciences, Bogota, Colombia; National School of Public Health (Prof R Sarmiento-Suárez MPH), Carlos III Health Institute, Madrid, Spain; Department of Geriatrics and Long Term Care (B Sathian PhD), Hamad Medical Corporation, Doha, Qatar; Faculty of
Health \& Social Sciences (B Sathian PhD), Bournemouth University, Bournemouth, UK; GSK Biologicals, Wavre, Belgium (M Savic PhD); Department of Epidemiology (Prof D Saxena PhD), Indian Institute of Public Health, Gandhinagar, India; Market Access (M Saylan MD), Bayer, Istanbul, Turkey; Unit for Hypertension and Cardiovascular Disease (Prof A E Schutte PhD), North-West University, Potchefstroom, South Africa; Oral Diagnosis, Digital Health and Health Services Research (Prof F Schwendicke PhD), Charité University Medical Center Berlin, Berlin, Germany; Department of Medical Statistics,

Epidemiology and Medical Informatics (M Sekerija PhD), University of Zagreb, Zagreb, Croatia; Department of Epidemiology and Prevention of Chronic Noncommunicable Diseases (M Sekerija PhD), Croatian Institute of Public Health, Zagreb, Croatia; Emergency Department (S Senthilkumaran MD), Manian Medical Centre, Erode, India; Environmental Health Associates LLC, Englewood, CO, USA (B Serdar PhD); Center for Biomedical Information Technology (F Sha PhD), Shenzhen Institutes of Advanced Technology, Shenzhen, China; Public Health Division (A A Shaheen PhD), An-Najah National University, Nablus, Palestine; Independent Consultant, Karachi, Pakistan (M A Shaikh MD); Faculty of Caring Science, Work Life, and Social Welfare (M Shamsizadeh MSc), University of Borås, Borås, Sweden; Department of Community Medicine (M Shannawaz PhD), BLDE University, Vijayapur, India; University School of Management and Entrepreneurship (R Sharma PhD), Delhi Technological University, Delhi, India; Centre for Medical Informatics (Prof A Sheikh MD), University of Edinburgh, Edinburgh, UK; College of Medicine (Prof J Shin MD), Yonsei University, Seoul, South Korea; Finnish Institute of Occupational Health, Helsinki, Finland (R Shiri PhD); School of Health (S Siabani PhD), University of Technology Sydney, Sydney, NSW, Australia; Medical Research Agency, Warsaw, Poland (R Sierpinski PhD); School of Medicine (R Sierpinski PhD), Cardinal Wyszynski University, Warsaw, Poland; Department of Psychology (Prof I D Sigfusdottir PhD, R Sigurvinsdottir PhD), Reykjavik University, Reykjavik, Iceland; Department of Physical Education

(Prof D A S Silva PhD), Federal University of Santa Catarina, Florianopolis, Brazil; Department of Law, Economics, Management and Quantitative Methods (Prof B Simonetti PhD), University of Sannio, Benevento, Italy; WSB University, Gdańsk, Poland (Prof B Simonetti PhD); Medicine Service (Prof J A Singh MD), US Department of Veterans Affairs (VA), Birmingham, AL, USA; Menzies Institute for Medical Research (A Singh MTech), University of Tasmania, Hobart, TAS, Australia; Global Patient Outcome and Real World Evidence (A Singh MTech), Eli Lilly and Company, Indianapolis, IN, USA; Department of Epidemiology (D N Sinha PhD), School of Preventive Oncology, Patna, India; Department of Epidemiology (D N Sinha PhD), Healis Sekhsaria Institute for Public Health, Mumbai, India; Department of Ophthalmology (E Skiadaresi MD), Hywel Dda University Health Board, Llanelli, UK; Department of Sports Science and Clinical Biomechanics (Prof S T Skou PhD), University of Southern Denmark, Odense, Denmark; Department of Physiotherapy and Occupational Therapy (Prof S T Skou PhD), Næstved-Slagelse-Ringsted Hospitals, Slagelse, Denmark; Department No.16 (V Y Skryabin MD), Laboratory of Genetics and Genomics (Prof M S Zastrozhin PhD), Moscow Research and Practical Centre on Addictions, Moscow, Russia; Department of Endocrinology and Diabetes (Prof E Sobngwi PhD), Yaoundé Central Hospital, Yaoundé, Cameroon; Nursing Care Research Center (A Soheili PhD), Semnan University of Medical Sciences, Semnan, Iran; Department of Infectious Diseases (A Sokhan PhD), Kharkiv National Medical University, Kharkiv, Ukraine; Demography and Population Studies Department (O D Somefun PhD), University of Witwatersrand, Johannesburg, South Africa; Centro de Investigación Biomédica en Red Enfermedades Respiratorias (CIBERES), Madrid, Spain (Prof J B Soriano MD); Department of Research Development (S Soshnikov PhD), Federal Research Institute for Health Organization and Informatics of the Ministry of Health (FRIHOI), Moscow, Russia (A V Korotkova PhD); Hull York Medical School (I N Soyiri PhD), University of Hull, Hull City, UK; Division of Community Medicine (C T Sreeramareddy MD), International Medical University, Kuala Lumpur, Malaysia; David Geffen School of Medicine (V Srinivasan MPH), University of California Los Angeles, Los Angeles, CA, USA; Centre for Global Health Inequalities Research (CHAIN) 
(K Sripada PhD), Department of Neuromedicine and Movement Science (Prof T J Steiner PhD, Prof L J Stovner PhD), Norwegian University of Science and Technology, Trondheim, Norway; Department of Primary Care and Public Health (Prof N Steel PhD), University of East Anglia, Norwich, UK; Occupational and Environmental Medicine Department (L Stockfelt PhD), University of Gothenburg, Gothenburg, Sweden; Department of Neurology and Clinical Neurophysiology (Prof L J Stovner PhD), St. Olavs Hospital, Trondheim, Norway; Schiller Institute (Prof K Straif PhD), Boston College, Boston, MA, USA; Department of Population Health (Prof S Stranges MD), Luxembourg Institute of Health, Strassen, Luxembourg; Department of Statistics (R Suliankatchi Abdulkader MD), Manonmaniam Sundaranar University, Abishekapatti, Tirunelveli, India; Pediatric Services (I Sultan MD), King Hussein Cancer Center, Amman, Jordan; Pediatrics Department (I Sultan MD), University of Jordan, Amman, Jordan; Delos Labs (C B Swope MPH, R Vaicekonyte MSc), Delos Living, New York, NY, USA; Department of Criminology, Law, and Society (Prof B L Sykes PhD), University of California Irvine, Irvine, CA, USA Minister of Health (L Szumowski MD), Institute of Cardiology Poland, Warsaw, Poland; Department of Medicine (Prof R Tabarés-Seisdedos PhD), University of Valencia, Valencia, Spain; School of Social Work (Prof K M Tabb PhD), University of Illinois, Urbana, IL, USA; Cancer Control Center (T Tabuchi MD), Osaka International Cancer Institute, Osaka, Japan; Department of Pharmacy (B W Taddele MPH), Arbaminch College of Health Sciences, Arbaminch, Ethiopia; Institute of Public Health, Lahore, Pakistan (Prof Z Tahir PhD); International Comion on Occupational Health, Monteporzio Catone, Rome, Italy (Prof J S Takala DSc); Africa Health Research Institute, Berea, South Africa (Prof F C Tanser PhD); Department of Population Science and Human Resource Development (Prof M I Tareque PhD), University of Rajshahi, Rajshahi, Bangladesh; Research and Development Center for Humanities and Health Management (I U Tarigan PhD), National Institute of Health Research \& Development, Jakarta, Indonesia; University Institute Egas Moniz, Monte da Caparica, Portugal (Prof N Taveira PhD); Research Institute for Medicines (Prof N Taveira PhD), University of Lisbon, Lisbon, Portugal; Division of Biostatistics and Epidemiology (B Thakur PhD), Texas Tech University, El Paso, TX, USA; Department of Public Health and Community Medicine (Prof K R Thankappan MD), Central University of Kerala, Kasaragod, India; Department of Endocrinology, Diabetes and Metabolism (Prof N Thomas PhD), Department of Nephrology (Prof S Varughese FRCP), Christian Medical College and Hospital (CMC), Vellore, India; Timiryazev Institute of Plant Physiology (M V Titova PhD), Russian Academy of Sciences, Moscow, Russia; Agency for Health Technology Assessment and Tariff System, Warsaw, Poland (R Topor-Madry PhD); Nutritional Epidemiology Research Team (EREN) (M Touvier PhD), National Institute for Health and Medical Research (INSERM), Paris, France; Health, Medicine and Human Biology (M Touvier PhD), Sorbonne Paris Nord University, Bobigny, France; Modestum LTD, London, UK (M R Tovani-Palone PhD); Department of Health Economics (B X Tran PhD), Hanoi Medical University, Hanoi, Vietnam; Mbarara University of Science and Technology, Mbarara, Uganda (A C Tsai MD); Department of Medicine (Prof A Tsatsakis DSc), Laboratory of Toxicology (C Vardavas PhD), University of Crete, Heraklion, Greece; Department of Microbiology (I Ullah PhD), Iqra National University, Peshawar, Pakistan; TB Culture Laboratory (I Ullah PhD), Mufti Mehmood Memorial Teaching Hospital, Dera Ismail Khan, Pakistan; Department of Community Medicine (C D Umeokonkwo MPH), Alex Ekwueme Federal University Teaching Hospital Abakaliki, Abakaliki, Nigeria; School of Government (E A Undurraga PhD), Pontifical Catholic University of Chile, Santiago, Chile; Schneider Institutes for Health Policy (E A Undurraga PhD), Brandeis University, Waltham, MA, USA; Amity Institute of Biotechnology (E Upadhyay PhD), Amity University Rajasthan, Jaipur, India; Velez Sarsfield Hospital, Buenos Aires, Argentina (Prof P R Valdez MEd); UKK Institute, Tampere, Finland (Prof T J Vasankari MD); Department of Statistics (Prof A N Vasconcelos PhD), University of Brasilia, Brasilia, Brazil; Psychosocial Injuries Research Center (Y Veisani PhD), Ilam University of Medical Sciences, Ilam, Iran; Raffles Neuroscience Centre (Prof N Venketasubramanian MBBS), Raffles Hospital, Singapore;
Department of Neurology (S Vidale MD), Infermi Hospital, Rimini, Italy; Department of Neurology \& Stroke Unit (S Vidale MD), Sant'Anna Hospital, Como, Italy; Occupational Health Unit (Prof F S Violante MD), Sant'Orsola Malpighi Hospital, Bologna, Italy; Department of Pediatric Endocrinology (R Vukovic PhD), Mother and Child Healthcare Institute of Serbia "Dr Vukan Cupic", Belgrade, Serbia; Foundation University Medical College (Prof Y Waheed PhD), Foundation University Islamabad, Islamabad, Pakistan; Department of Epidemiology and Biostatistics (J Wei PhD), Department of Neurology (M T Wallin MD), George Washington University, Washington, DC, USA; School of Health Sciences (F Wang MA, X G Zhao PhD), Department of Epidemiology and Biostatistics (Y Wang BSA, Prof C Yu PhD), Global Health Institute (Prof C Yu PhD), School of Medicine (Z Zhang PhD), Wuhan University, Wuhan, China; Division of Social Science (H Wang DipSocSc), Hong Kong University of Science and Technology, Hong Kong, China; Cardiology Department (Prof R G Weintraub MB), Royal Children's Hospital, Melbourne, VIC, Australia; Leonard Davis Institute of Health Economics (J Weiss MA), Population Studies Center (J Weiss MA), University of Pennsylvania, Philadelphia, PA, USA; Department of Physical Therapy (T Wiangkham PhD), Naresuan University, Phitsanulok, Thailand; Department of Community Medicine (N D Wickramasinghe MD), Rajarata University of Sri Lanka, Anuradhapura, Sri Lanka; Department of Medicine

(Prof T Wijeratne MD), University of Rajarata, Saliyapura Anuradhapuraya, Sri Lanka; NIHR Biomedical Research Centre (Prof C D A Wolfe MD), Guy's and St.Thomas' Hospital and Kings College London, London, UK; Department of Orthopaedics (Prof A Wu MD), Wenzhou Medical University, Wenzhou, China; Department of Behavior and Operation Management (Y Xie MD), Beijing Advanced Innovation Center for Big Data-based Precision Medicine, Beijing, China; School of Medicine (Prof G Xu MD), Nanjing University, Nanjing, China; Clinical Cancer Research Center (S Yahyazadeh Jabbari MD), Milad General Hospital, Tehran, Iran; Research and Development Center for Health Services (Prof K Yamagishi MD), University of Tsukuba, Tsukuba, Japan; Pediatrics Department (C S Yilgwan MD), University of Jos, Jos, Nigeria; Department of Pediatrics (C S Yilgwan MD), Jos University Teaching Hospital, Jos, Nigeria; Department of Neuropsychopharmaology (N Yonemoto MPH), National Center of Neurology and Psychiatry, Kodaira, Japan; Department of Public Health (N Yonemoto MPH), Juntendo University, Tokyo, Japan; Department of Health Policy and Management (Prof M Z Younis PhD), Jackson State University, Jackson, MS, USA; School of Medicine (Prof M Z Younis PhD), Tsinghua University, Beijing, China; Department of Immunology (T P Younker BS), University of Pittsburgh, Pittsburgh, PA, USA; School of Public Health and Management (Y Yu MS), Hubei University of Medicine, Shiyan, China; Department of Pharmaceutics (S Zaidi PhD), Dow University of Health Sciences, Karachi, Pakistan; Addictology Department (Prof M S Zastrozhin PhD), Russian Medical Academy of Continuous Professional Education, Moscow, Russia; Victorian Comprehensive Cancer Centre, Melbourne, VIC, Australia (J Zhang MD); Hubei Province Key Laboratory of Occupational Hazard Identification and Control (Y Zhang PhD), School of Public Health (Y Zhang PhD), Wuhan University of Science and Technology, Wuhan, China; School of Biology and Pharmaceutical Engineering (X G Zhao PhD), Wuhan Polytechnic University, Wuhan, China; Global Strategy and Advocacy Unit (B Zheleva MBA), Children's HeartLink, Minneapolis, MN, USA; Department of Epidemiology, Human Genetics, and Environmental Sciences (C Zhu MPH), University of Texas Health Science Center at Houston, Houston, TX, USA.

\section{Declaration of interests}

$\mathrm{R}$ Ancuceanu reports receiving consultancy and speakers' fees from various pharmaceutical companies. E Beghi reports grants from Italian Ministry of Health and Swedish Orphan Biovitrum; and personal fees from Arvelle Therapeutics, outside the submitted work. M Bell reports grants from US Environmental Protection Agency, National Institutes of Health (NIH), and Wellcome Trust Foundation, during the conduct of this work; and Honorarium and travel reimbursement from the NIH (for review of grant proposals), American Journal of Public Health (participation as editor), Global Research Laboratory and Seoul National 
University, Royal Society, London UK, Ohio University, Atmospheric Chemistry Gordon Research Conference, Johns Hopkins Bloomberg School of Public Health, Arizona State University, Ministry of the Environment Japan, Hong Kong Polytechnic University, University of Illinois-Champaign, and the University of Tennessee-Knoxville, outside the submitted work. PS Briant and L Haile report personal fees from WHO, outside of the submitted work. V Jha reports grants from Baxter Healthcare, GlaxoSmithKline, Zydus Cadilla, Biocon, and NephroPlus, outside the submitted work. S Lorkowski reports personal fees from Akcea Therapeutics, Amedes, Amgen, Berlin-Chemie, Boehringer Ingelheim, Daiichi Sankyo, Merck Sharp and Dohme, Novo Nordisk, Sanofi-Aventis, Synlab, Unilever, and Upfield; and non-financial support from Preventicus, outside the submitted work. J Mosser reports grants from Bill and Melinda Gates Foundation, during the conduct of this work. S Nomura reports grants from the Ministry of Education, Culture, Sports, Science, and Technology. C Pond reports personal fees from Nutricia, during the conduct of this work; grants from the National Medical Research council in relation to dementia; and travel grants and remuneration related to education of primary care professionals in relation to dementia, outside of this work. M Postma reports grants from BioMerieux, WHO, EU, Foundation for Innovative New Diagnostics, Antilope, Pangkalan Data Pendidikan Tinggi, Indonesia Endowment Fund for Education (Lembaga Pengelola Dana Pendidikan), Bayer, and Budi, personal fees from Quintiles, Novartis, and Pharmerit; grants and personal fees from IMSQuintiles, Bristol-Myers Squibb, AstraZeneca, Seqirus, Sanofi, Merck Sharp and Dohme, GlaxoSmithKline, Pfizer, Boehringer Ingelheim, and Novavax; stocks from Ingress Health, and Pharmacoeconomics Advice Groningen; and acting as advisor to Asc Academics, all outside the submitted work. E Pupillo reports grants from Agenzia Italiana del Farmaco (Italian Medicines Agency), outside the submitted work. A Schutte reports personal fees from Omron Healthcare, Takeda, Servier, Novartis, and Abbott, outside the submitted work. M Shrime reports grants from Mercy Ships and Damon Runyon Cancer Research Foundation, outside the submitted work. J Singh reports personal fees from Crealta-Horizon, Medisys, Fidia, UBM LLC, Trio health, Medscape, WebMD, Clinical Care options, Clearview healthcare partners, Putnam associates, Spherix, Practice Point communications, the National Institutes of Health and the American College of Rheumatology; personal fees from Simply Speaking; stock options in Amarin pharmaceuticals and Viking pharmaceuticals; membership in the steering committee of Outcome Measures in Rheumatology, an international organization that develops measures for clinical trials and receives arm's length funding from 12 pharmaceutical companies, membership in the Food and Drug Administration's Arthritis Advisory Committee, membership in the Veterans Affairs Rheumatology Field Advisory Committee; and non-financial support from University of Alabama at Birmingham Cochrane Musculoskeletal Group Satellite Center on Network Meta-analysis, outside the submitted work. J Stanaway reports grants from Bill and Melinda Gates Foundation, during the conduct of this work. D Stein reports personal fees from Lundbeck and Sun, outside the submitted work. C Swope reports working for a private sector organization, Delos Living. R Uddin reports travel and accommodation reimbursement from Deakin University Institute for Physical Activity and Nutrition, outside the submitted work. $\mathrm{R}$ Vaicekonyte reports working for a private sector organisation, Delos Living. All other authors declare no competing interests.

\section{Acknowledgments}

L Abreu acknowledges support from Coordenação de Aperfeiçoamento de Pessoal de Nível Superior, Brazil, Finance Code 001 and Conselho Nacional de Desenvolvimento Científico e Tecnológico. A Agrawal acknowledges support from the Wellcome DBT Senior Fellowship IA/CPHS/14/1/501489. S Aljunid acknowledges the Department of Health Policy and Management, Faculty of Public Health, Kuwait University and International Centre for Casemix and Clinical Coding, Faculty of Medicine, National University of Malaysia for the approval and support to participate in this research project. A Badawi acknowledges support from the Public Health Agency of Canada. J Carrero acknowledges support from the Swedish Research Council (2019-01059). F Carvalho acknowledges UID/MULTI/04378/2019 and UID/QUI/50006/2019 support with funding from FCT/MCTES through national funds. A Cohen acknowledges support from the Health Effects Institute,
Boston, MA, USA. V Costa acknowledges grant

(SFRH/BHD/110001/2015), received by Portuguese national funds through Fundação para a Ciência e Tecnologia, IP, under the Norma Transitória DL57/2016/CP1334/CT0006. K Deribe acknowledges a grant from the Wellcome Trust (grant number 201900/Z/16/Z) as part of his International Intermediate Fellowship. M Freitas acknowledges the financial support from the European Union (FEDER funds through COMPETE POCI-01-0145-FEDER-029248) and National Funds (Fundação para a Ciência e Tecnologia) through project PTDC/NANMAT/29248/2017. P Hoogar acknowledges Centre for Bio Cultural Studies, Manipal Academy of Higher Education, Manipal and Centre for Holistic Development and Research, Kalaghatagi. B-F Hwang was supported by China Medical University (CMU108-MF-95), Taichung, Taiwan. M Jakovljevic acknowledges the Serbian part of this GBD Project related contribution was co-funded through Grant OI175014 of the Ministry of Education Science and Technological Development of the Republic of Serbia. C Kieling is a Conselho Nacional de Desenvolvimento Científico e Tecnológico (CNPq; a Brazilian public funding agency) researcher and a UK Academy of Medical Sciences Newton Advanced Fellow. Y J Kim acknowledges support from the Research Management Centre, Xiamen University Malaysia (XMUMRF/2018-C2/ITCM/0001). K Krishan is supported by a DST PURSE grant and UGC Center of Advanced Study (CAS II) awarded to the Department of Anthropology, Panjab University, Chandigarh, India. M Kumar is supported by FIC/NIH K43 TW010716. B Lacey acknowledges support from the National Institute for Health Research Oxford Biomedical Research Centre and the British Heart Foundation Centre of Research Excellence, Oxford. T Lallukka is supported by the Academy of Finland (Grant \#319200). I Landires is member of the Sistema Nacional de Investigación, which is supported by the Secretaría Nacional de Ciencia tecnología e Innovación, Panama. S Lorkowski is funded by the German Federal Ministry of Education and Research (nutriCARD, grant agreement number 01EA1808A). J McGrath acknowledges support from the Danish National Research Foundation (Niels Bohr Professorship). W Mendoza acknowledges employment with Population and Development at the United Nations Population Fund, Country Office in Peru, which does not necessarily endorse this study. U Mueller acknowledges funding from the German National Cohort Study BMBF grant \# 01ER1801D. S Nomura acknowledges the Ministry of Education, Culture, Sports, Science, and Technology of Japan (18K10082). G Patton is supported by an National Health and Medical Research Council Fellowship. M Phillips acknowledges partial support from the National Natural Science Foundation of China (NSFC, No 81371502 and 81761128031). M Pinheiro acknowledges FCT Fundação para a Ciência e Tecnologia for funding through program DL 57/2016, Norma Transitória. A Raggi, D Sattin, and S Schiavolin acknowledge support by a grant from the Italian Ministry of Health (Ricerca Corrente, Fondazione Istituto Neurologico C Besta, Linea 4 - Outcome Research: dagli Indicatori alle Raccomandazioni Cliniche). D Ribeiro acknowledges support from The Sir Charles Hercus Health Research Fellowship Health Research Council of New Zealand (18/111). A Ribeiro acknowledges partial support by Conselho Nacional de Desenvolvimento Científico e Tecnológico (310679/2016-8 and IATS: 465518/2014-1) and by Fundação de Amparo à Pesquisa do Estado de Minas Gerais (PPM-00 428-17). P Sachdev acknowledges funding support from NHMRC Australia. A Samy received a fellowship from the Egyptian Fulbright Mission program. M Santric-Milicevic acknowledges the Ministry of Education, Science and Technological Development of the Republic of Serbia (Contract No 175087). S Islam received funding from the National Heart Foundation of Australia and Deakin University. A Sheikh acknowledges Health Data Research UK. K Shibuya acknowledges the Japan Ministry of Education, Culture, Sports, Science and Technology. J Soriano acknowledges support from Centro de Investigación en Red de Enfermedades Respiratorias, Instituto de Salud Carlos III, Madrid, Spain. R Tabarés-Seisdedos was supported in part by grant PI17/00719 from ISCIII-FEDER. S Tadakamadla acknowledges support from National Health and Medical Research Council Early Career Fellowship, Australia. M Tonelli acknowledges the David Freeze research chair in health services research at the University of Calgary. See the component GBD 2019 capstone papers for acknowledgements specific to each part of the study. 


\section{References}

1 Murray CJL, Callender CSKH, Kulikoff XR, et al. Population and fertility by age and sex for 195 countries and territories, 1950-2017 a systematic analysis for the Global Burden of Disease Study 2017. Lancet 2018; 392: 1995-2051.

2 UN Millennium Project. Investing in development: a practical plan to achieve the millennium development goals. New York, NY: United Nations Development Programme, 2005.

3 GBD 2019 Demographics Collaborators. Global age-sex-specific fertility, mortality, healthy life expectancy (HALE), and population estimates in 204 countries and territories, 1950-2019: a comprehensive demographic analysis for the Global Burden of Disease Study 2019. Lancet 2020; 396: 1160-203.

4 Murray CJL, Ortblad KF, Guinovart C, et al. Global, regional, and national incidence and mortality for HIV, tuberculosis, and malaria during 1990-2013: a systematic analysis for the Global Burden of Disease Study 2013. Lancet 2014; 384: 1005-70.

5 Komatsu R, Korenromp EL, Low-Beer D, et al. Lives saved by Global Fund-supported HIV/AIDS, tuberculosis and malaria programs: estimation approach and results between 2003 and end-2007. BMC Infect Dis 2010; 10: 109.

6 Institute for Health Metrics and Evaluation. Financing global health 2018: countries and programs in transition. Seattle, WA: Institute for Health Metrics and Evaluation, 2019.

7 GBD 2019 Diseases and Injuries Collaborators. Global burden of 369 diseases and injuries in 204 countries and territories, 1990-2019: a systematic analysis for the Global Burden of Disease Study 2019. Lancet 2020; 396: 1204-22.

8 GBD 2019 Universal Health Coverage Collaborators. Measuring universal health coverage based on an index of effective coverage of health services in 204 countries and territories, 1990-2019: a systematic analysis for the Global Burden of Disease Study 2019. Lancet 2020; 396: 1250-84.

9 United Nations. SDG Indicators-Global indicator framework for the Sustainable Development Goals and targets of the 2030 Agenda for Sustainable Development. https://unstats.un.org/sdgs/ indicators/indicators-list/ (accessed Jan 27, 2020).

10 Dieleman JL, Cao J, Chapin A, et al. US health care spending by payer and health condition, 1996-2016. JAMA 2020; 323: 863-84.

11 United Nations General Assembly. Political declaration of the third high-level meeting of the General Assembly on the prevention and control of non-communicable diseases. New York, NY: United Nations, 2018.

12 Nugent R, Bertram MY, Jan S, et al. Investing in non-communicable disease prevention and management to advance the Sustainable Development Goals. Lancet 2018; 391: 2029-35.

13 WHO. Noncommunicable diseases. June 1, 2018. https://www.who. int/news-room/fact-sheets/detail/noncommunicable-diseases (accessed Jan 27, 2020).

14 Cottingham MD, Kalbaugh CA, Fisher JA. Tracking the pharmaceutical pipeline: clinical trials and global disease burden. Clin Transl Sci 2014; 7: 297-99.

15 Fisher JA, Cottingham MD, Kalbaugh CA. Peering into the pharmaceutical "pipeline": investigational drugs, clinical trials, and industry priorities. Soc Sci Med 2015; 131: 322-30.

16 Long G. The biopharmaceutical pipeline: innovative therapies in clinical development. Boston, MA: Analysis Group, 2017.
17 National Institutes of Health. Estimates of funding for various research, condition, and diseases categories (RCDC). 2019. https://report.nih.gov/categorical_spending.aspx (accessed Jan 27, 2020).

18 GBD 2019 Risk Factors Collaborators. Global burden of 87 risk factors in 204 countries and territories, 1990-2019: a systematic analysis for the Global Burden of Disease Study 2019. Lancet 2020; 396: 1223-49.

19 WHO. WHO framework convention on tobacco control. Geneva: World Health Organization, 2003.

20 Maximova K, Raine KD, Czoli C, et al. Monitoring progress toward United Nations commitments: characteristics of Canadian legislation to promote tobacco control, physical activity and healthy eating. A descriptive study. CMAJ Open 2019; 7: E745-53.

21 Yach D, McKee M, Lopez AD, Novotny T. Improving diet and physical activity: 12 lessons from controlling tobacco smoking. BMJ 2005; 330: 898-900.

22 Afshin A, Forouzanfar MH, Reitsma MB, et al. Health effects of overweight and obesity in 195 countries over 25 years. $N$ Engl J Med 2017; 377: 13-27.

23 Pineda E, Sanchez-Romero LM, Brown M, et al. Forecasting future trends in obesity across Europe: the value of improving surveillance. Obes Facts 2018; 11: 360-71.

24 Lopez AD, Adair T. Is the long-term decline in cardiovasculardisease mortality in high-income countries over? Evidence from national vital statistics. Int J Epidemiol 2019; 48: 1815-23.

25 Vollset S, Goren E, Yuan C-W, et al. Fertility, mortality, migration, and population scenarios for 195 countries and territories from 2017 to 2100: a forecasting analysis for the Global Burden of Disease Study. Lancet 2020; 396: 1285-306.

26 Graetz N, Woyczynski L, Wilson KF, et al. Mapping disparities in education across low- and middle-income countries. Nature 2020; 577: 235-38.

27 Lozano R, Fullman N, Abate D, et al. Measuring progress from 1990 to 2017 and projecting attainment to 2030 of the health-related Sustainable Development Goals for 195 countries and territories: a systematic analysis for the Global Burden of Disease Study 2017. Lancet 2018; 392: 2091-138.

28 The Kremlin. The President signed executive order on national goals and strategic objectives of the Russian Federation through to 2024. May 7, 2018. http://en.kremlin.ru/events/president/ news/57425 (accessed Jan 27, 2020).

29 Bricker D, Ibbitson J. Empty planet: the shock of global population decline. New York, NY: Crown, 2019.

30 Swee-Hock S. Population policies and programmes in Singapore, 2nd edn. Singapore: ISEAS Yosof Ishak Institute, 2016.

31 Lee C. Parents of children 5 and under get 100,000 won monthly allowance in South Korea. Sep 19, 2018. http://annx.asianews. network/content/parents-children-5-and-under-get-100000-wonmonthly-allowance-south-korea-82056 (accessed Jan 27, 2020).

32 The Government of Japan. Abenomics. https://www.japan.go.jp/ abenomics/index.html (accessed Aug 29, 2019).

33 Lee M, Lin Y-H. Transition from anti-natalist to pro-natalist policies in Taiwan. In: Rindfuss R, Choe M, eds. Low fertility, insitutions, and their policies: variations across industrialized countries. Honolulu, HI: Springer, 2016: 259-81.

(C) 2020 Elsevier Ltd. All rights reserved. 